\title{
Two-Photon Absorption Laser Induced Fluorescence Measurements of Neutral Density in Helicon Plasma
}

\author{
Matthew E. Galante \\ West Virginia University
}

Follow this and additional works at: https://researchrepository.wvu.edu/etd

\section{Recommended Citation \\ Galante, Matthew E., "Two-Photon Absorption Laser Induced Fluorescence Measurements of Neutral Density in Helicon Plasma" (2013). Graduate Theses, Dissertations, and Problem Reports. 3653. https://researchrepository.wvu.edu/etd/3653 \\ This Dissertation is protected by copyright and/or related rights. It has been brought to you by the The Research Repository @ WVU with permission from the rights-holder(s). You are free to use this Dissertation in any way that is permitted by the copyright and related rights legislation that applies to your use. For other uses you must obtain permission from the rights-holder(s) directly, unless additional rights are indicated by a Creative Commons license in the record and/ or on the work itself. This Dissertation has been accepted for inclusion in WVU Graduate Theses, Dissertations, and Problem Reports collection by an authorized administrator of The Research Repository @ WVU. For more information, please contact researchrepository@mail.wvu.edu.}




\title{
Two-Photon Absorption Laser Induced Fluorescence Measurements of Neutral Density in Helicon Plasma
}

\author{
Matthew E. Galante \\ Dissertation submitted to the College of Arts and Sciences \\ at West Virginia Univeristy \\ in partial fulfillment of the requirements \\ for the degree of \\ Doctor of Philosophy \\ in \\ Physics
}

Earl E. Scime, Ph.D. Chair

Paul A. Cassak, Ph.D.

Timothy N. Good, Ph.D.

Fred L. King, Ph.D.

H. Arthur Weldon, Ph.D.

Department of Physics

Morgantown, West Virginia

2013

Keywords: helicon, laser induced fluorescence, neutral density, neutral depletion Copyright 2013 Matthew E. Galante 


\title{
ABSTRACT \\ Two-Photon Absorption Laser Induced Fluorescence Measurements of Neutral Density in Helicon Plasma
}

\begin{abstract}
Matthew E. Galante
Neutral particles play a critical role in plasma experiments. They simultaneously act as a source of particles and a sink of momentum and energy. Also, they act as an intermediary between the plasma and surrounding material walls. However, few methods exist to make localized, direct neutral density measurements. A new diagnostic, based on two-photon absorption laser induced fluorescence (TALIF), capable of making direct ground state measurement is developed. A high intensity $\left(5 \mathrm{MW} / \mathrm{cm}^{2}\right)$, narrow bandwidth $\left(0.1 \mathrm{~cm}^{-1}\right)$ laser is used to directly probe the ground state of neutral hydrogen, deuterium and krypton. The diagnostic represents an improvement over traditional laser induced fluorescence in that it can be used to measure the spatial profile from a single porthole, and it can be operated in a Doppler-free configuration. The system is tested and calibrated in a low temperature helicon plasma source. Calculations show the system has sufficient performance to achieve an acceptable signal-to-noise ratio in a high temperature fusion plasma. Development of the diagnostic is presented in this work along with both steady-state and time-resolved TALIF measurements in helicon plasma.
\end{abstract}




\section{Acknowledgement}

First, I would like to thank my advisor, Dr. Earl Scime, for all his help and support. Thank you for all the opportunities you have provided me and for all you have taught me. Thank you to Dr. Richard Magee, my partner in TALIF. Your knowledge and patience throughout the last $2+$ years of graduate school have been invaluable, and truly have made all the work we accomplished together possible.

Thank you to all the faculty and staff of the WVU Physics Department, especially Dr. Arthur Weldon and Dr. Paul Cassak. Thank you for having open doors, and never turning me aside, no matter however trivial my questions may have been.

Thank you to the other members of the Scime research group, Dr. Amy Keesee, Jerry Carr Jr., Justin Elfritz, Michael Lindon, Dustin McCarren, Stephanie Sears, and our under-

grads, Greg Lusk, Mark Soderholm, and Robert Vandervort. Thank you for all your help, especially my office mates, Justin Elfritz and Dustin McCarren.

Thank you to all the other fifth year graduate students, especially Matthew Beidler, Colin Komar, Katherine Rabidoux and Andrew Seymour. You are all my dear friends and without you I would not be where I am today.

Lastly, thank you to my family, especially my parents and my wife Elizabeth Galante. You push me to do my best everyday, and never let me get too down, or high, on myself.

This work was supported by US DOE grant DE-FC02-04ER54698. 
This work is dedicated to my wife, Elizabeth Galante. Without you, I would have never made it through this, and I promise, there's no grad school after grad school. 


\section{Contents}

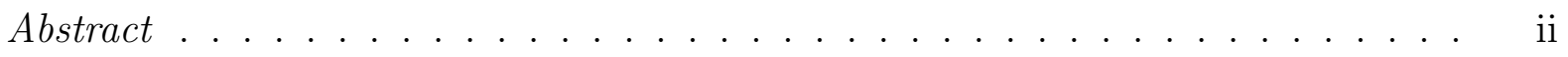

Acknowledgements ........................... ii

Dedication .......................... iv

Table of Contents............................... v

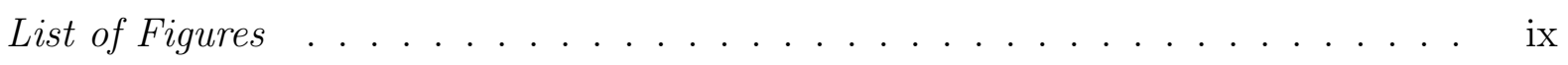

1 Introduction 1

1.1 Neutral density in helicon plasma sources . . . . . . . . . . . . . . . . 2

1.2 Neutral density in fusion devices . . . . . . . . . . . . . . 8

1.2 .1 Charge-Exchange . . . . . . . . . . . . . . . . 9

$1.2 .2 \quad$ H Mode . . . . . . . . . . . . . . . . . . . . . . . . . . . . . 13

1.2 .3 Neutral Retention/Recycling . . . . . . . . . . . . . . . . . 18

1.3 Neutral density measurement techniques . . . . . . . . . . . . . . . 19

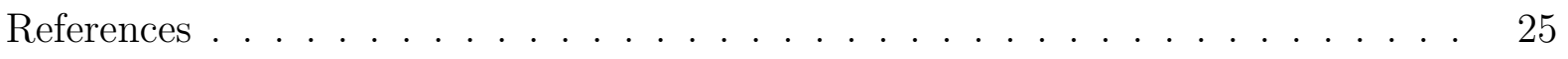

2 Helicons 31

2.1 Background . . . . . . . . . . . . . . . . . . . . . . 31

2.2 Physics. . . . . . . . . . . . . . . . . . . . . . . . . 33 
2.2.1 Lower Hybrid Resonance . . . . . . . . . . . . . . . . . 38

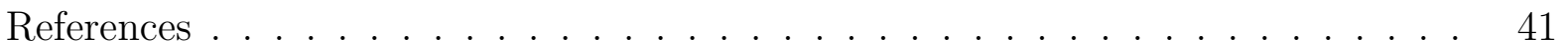

3 Experimental Apparatus $\quad 45$

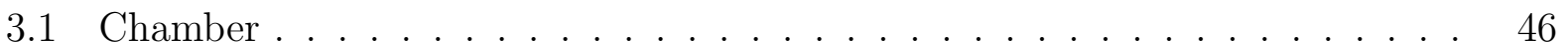

3.2 Vacuum system . . . . . . . . . . . . . . . . . 46

3.3 Magnetic Field . . . . . . . . . . . . . . . . . . . . 47

3.4 RF antenna and matching network . . . . . . . . . . . . . 50

3.5 Plasma Parameters . . . . . . . . . . . . . . . . . 52

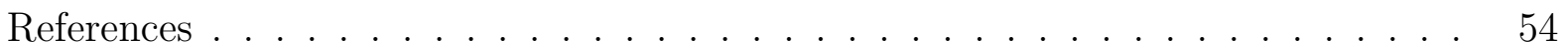

4 Diagnostics $\quad 55$

4.1 Optical Emission Spectroscopy . . . . . . . . . . . . . . . 55

4.2 Langmuir Probes . . . . . . . . . . . . . . . . . . . 58

4.2.1 Langmuir Probe Theory . . . . . . . . . . . . . . . . . 58

4.2.2 Langmuir Probe Design . . . . . . . . . . . . . . . . 60

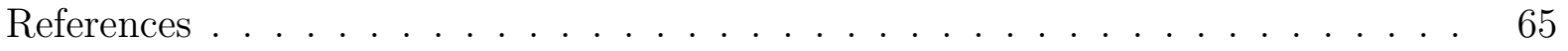

$5 \quad$ Two-Photon Absorption Laser Induced Fluorescence $\quad 67$

5.1 History . . . . . . . . . . . . . . . . . . 68

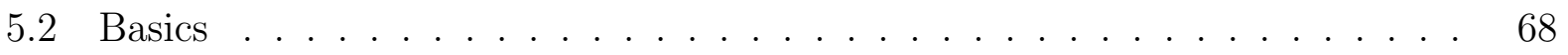

5.2.1 Transition Selection . . . . . . . . . . . . . . . 72

5.3 Line Broadening . . . . . . . . . . . . . . . . . . . . . . . 74

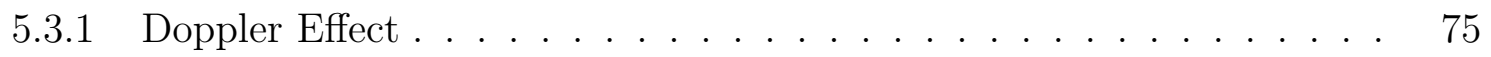

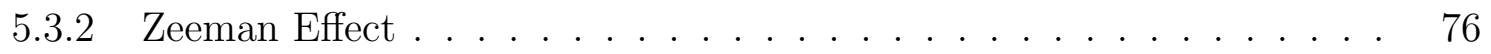

5.3.3 Pressure/Stark Effect . . . . . . . . . . . . . . . 80 
5.3.4 Saturation Broadening .................. 81

5.3.5 Isotopic Splitting . . . . . . . . . . . . . . . . . 82

5.4 TALIF Laser System . . . . . . . . . . . . . . . . . . . . 83

5.4.1 Harmonic Frequency Generation . . . . . . . . . . . . . . . 84

5.4 Laser System . . . . . . . . . . . . . . . . . . 86

5.4 .3 Collection Geometry ........................ 90

5.5 Data Acquisition and Signal Analysis . . . . . . . . . . . . . . . . 94

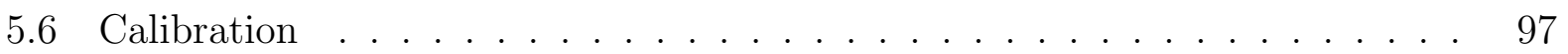

5.6.1 Hydrogen Calibration . . . . . . . . . . . . . . . . 99

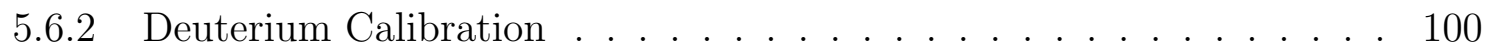

5.7 Signal-to-Noise . . . . . . . . . . . . . . . . . . . . 101

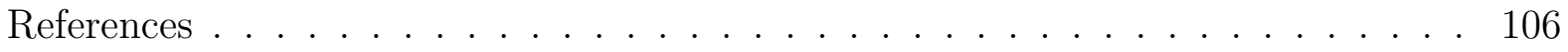

6 Steady-State Plasma TALIF Measurements $\quad 111$

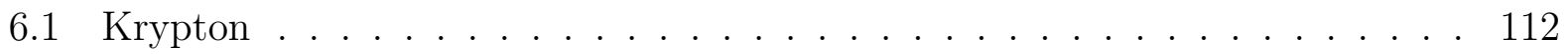

6.1.1 Parametric Scans in Krypton Plasma . . . . . . . . . . . . . . . . 112

6.1 .2 Axial Profiles in Krypton Plasma . . . . . . . . . . . . . . . 118

6.2 Hydrogen . . . . . . . . . . . . . . . . . . . . 122

6.2.1 Parametric Scans in Hydrogen Plasma . . . . . . . . . . . . . . . 122

6.2.2 Axial Profiles in Hydrogen Plasma . . . . . . . . . . . . . . . . 135

6.3 Mixed Species . . . . . . . . . . . . . . . . . . 136

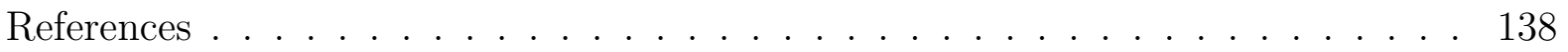

7 Time Resolved TALIF Measurements 141

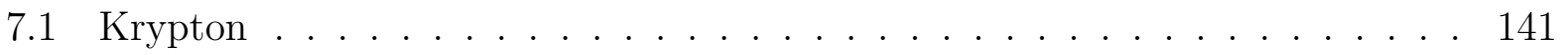


7.2 Hydrogen . . . . . . . . . . . . . . . . . . . . . . . 145

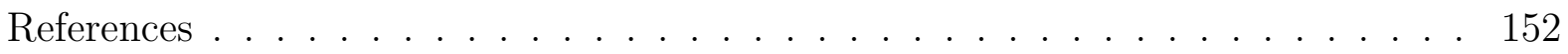

8 Summary of Results and Future Work 153

$\begin{array}{ll}\text { A CHEWIE Pulsing Scheme } & 157\end{array}$

A.1 Triggering Scheme . . . . . . . . . . . . . . . 157

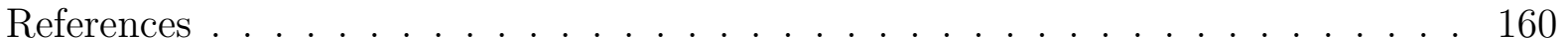

B Dye Recipe $\quad 161$

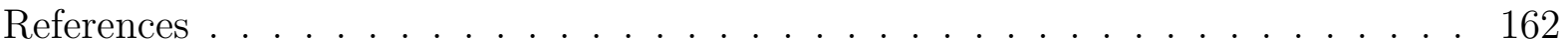




\section{List of Figures}

1.1 HELIX ion flow $\ldots \ldots \ldots \ldots \ldots \ldots \ldots \ldots \ldots \ldots \ldots \ldots \ldots$

1.2 Neutral Pumping . . . . . . . . . . . . . . . . . . . . . . . 7

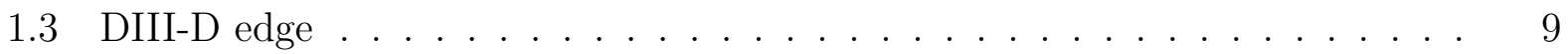

$1.4 \mathrm{H}$ rate coefficients . . . . . . . . . . . . . . . . . . . . . . . . . . 10

1.5 Neutral penetration length . . . . . . . . . . . . . . . . . . . . . . . . 12

1.6 Tokamak rotation rate . . . . . . . . . . . . . . . . . . . . . . . . . . 13

1.7 ASDEX confinement time . . . . . . . . . . . . . . . . . 15

$1.8 \mathrm{H}$ mode plasma density . . . . . . . . . . . . . . . . . . . . . . . . . . . 16

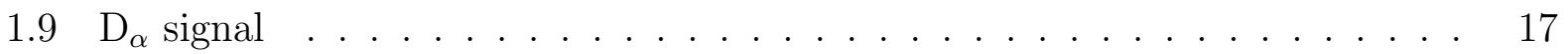

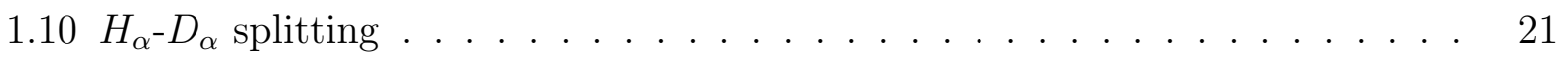

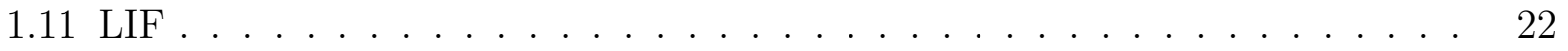

1.12 Hydrogen LIF schemes . . . . . . . . . . . . . . . . . . . . . . . 23

2.1 Helicon Plasma Density . . . . . . . . . . . . . . . . . . . . . . . . 34

2.2 Power absorption . . . . . . . . . . . . . . . . . . . . 36

2.3 Maxwellian electrons . . . . . . . . . . . . . . . . . . . . . . . . . . . 37

2.4 Lower hybrid resonance . . . . . . . . . . . . . . . . . . . . . . . . . . . . 39

2.5 Plasma production . . . . . . . . . . . . . . . . . . . . 40 


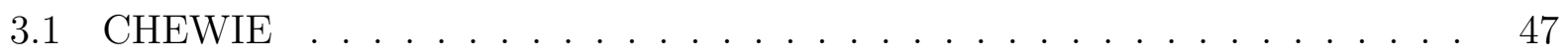

3.2 CHEWIE B-field . . . . . . . . . . . . . . . . . . 48

3.3 Axial Magnetic Field . . . . . . . . . . . . . . . . . . . . . 49

3.4 B field profile . . . . . . . . . . . . . . . . . . 50

3.5 CHEWIE Antenna ............................. 51

3.6 Matching network circuit . . . . . . . . . . . . . . . . 52

4.1 Czerny-Turner Spectrometer . . . . . . . . . . . . . . 56

$4.2 \mathrm{H}$ emission spectrum . . . . . . . . . . . . . . . . . . 57

4.3 Langmuir Probe Trace . . . . . . . . . . . . . . . . . . . 58

4.4 Langmuir probes . . . . . . . . . . . . . . . . . . . 61

4.5 Langmuir Probe Circuit . . . . . . . . . . . . . . . . . . . 62

4.6 Langmuir Probe Assembly . . . . . . . . . . . . . . . . . . . . . 62

4.7 Pulsed Langmuir Probe Time History . . . . . . . . . . . . . . . . . . 63

5.1 Two-photon absorption . . . . . . . . . . . . . . 70

$5.2 \quad L y_{\beta}$ splitting . . . . . . . . . . . . . . . . . . . . 72

5.3 H Ground State Transition . . . . . . . . . . . . . . . . . . . 72

5.4 H Partial Grotrian Diagram . . . . . . . . . . . . . . . . . 74

5.5 Doppler broadening . . . . . . . . . . . . . . . . 76

5.6 Zeeman transitions .......................... 77

5.7 CHEWIE Zeeman splitting . . . . . . . . . . . . . . . 78

5.8 Paschen-Back transitions . . . . . . . . . . . . . . . . . . 79

5.9 Paschen-Back splitting . . . . . . . . . . . . . . . . . 79

5.10 H-D-T $L y_{\beta}$ splitting . . . . . . . . . . . . . . . . . . . . . . . 82 
$5.11 \mathrm{Kr}$ isotope splitting . . . . . . . . . . . . . . . . . . . 83

5.12 Second Harmonic Generation _. . . . . . . . . . . . . . . . . 85

5.13 Sum-Frequency Generation . . . . . . . . . . . . . . 86

5.14 Dye laser layout . . . . . . . . . . . . . . . . . . . . . . . 87

5.15 Laser Temporal Shape . . . . . . . . . . . . . . . . . . . . . . 88

5.16 Beam Profile . . . . . . . . . . . . . . . . . . . . . . 89

5.17 Perpendicular collection . . . . . . . . . . . . . . . . . . . . . . 91

5.18 Confocal collection . . . . . . . . . . . . . . . . . . 92

5.19 Counter propagating collection . . . . . . . . . . . . . . . 93

5.20 Background Subtraction . . . . . . . . . . . . . . . . . . 95

$5.21 \mathrm{Kr}$ Saturation Energy . . . . . . . . . . . . . . . . . . . . . . . 99

6.1 Helicon core vs no core . . . . . . . . . . . . . . . . . . . . . . . . . . 112

6.2 Kr density vs power . . . . . . . . . . . . . . . . . . . 113

$6.3 \mathrm{Kr}$ density vs magnetic field . . . . . . . . . . . . . . . . . 114

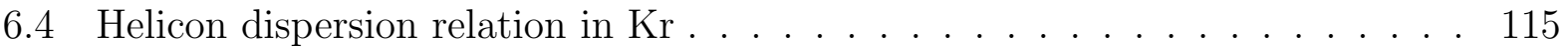

$6.5 \mathrm{Kr}$ temperature vs magnetic field . . . . . . . . . . . . . . . . . 115

$6.6 \mathrm{Kr}$ density vs magnetic field . . . . . . . . . . . . . . . . . . . . . 117

6.7 Radial $\mathrm{Kr}$ density vs field . . . . . . . . . . . . . . . . . . . . 118

6.8 Kr Lower Hybrid Scan . . . . . . . . . . . . . . . . . . . . . . . . . . 119

$6.9 \mathrm{Kr}$ density vs $\mathrm{rf} \ldots \ldots \ldots$. . . . . . . . . . . . . . . . . . . 119

6.10 Radial $\mathrm{Kr}$ ionization fraction . . . . . . . . . . . . . . . . . . 120

6.11 CHEWIE Sideways . . . . . . . . . . . . . . . . . . . . . . . 121

6.12 Axial Kr neutral density . . . . . . . . . . . . . . . . . . . . . . . 121 
$6.13 \mathrm{H}$ and $\mathrm{D}$ density vs power . . . . . . . . . . . . . . . 123

6.14 Neutral H density vs radius vs power . . . . . . . . . . . . . . . . . 124

$6.15 \mathrm{H}$ temperature vs radius vs power . . . . . . . . . . . . . 125

6.16 Two Temperature Distribution . . . . . . . . . . . . . . 126

$6.17 \mathrm{H}$ and $\mathrm{D}$ density vs magnetic field . . . . . . . . . . . . . 128

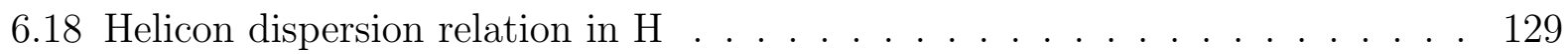

$6.19 \mathrm{H}$ and $\mathrm{D}$ density vs radius vs magnetic field . . . . . . . . . . . . 130

$6.20 \mathrm{H}$ and $\mathrm{D}$ density vs pressure . . . . . . . . . . . . . . . 131

6.21 Electron Skin Depth . . . . . . . . . . . . . . . . . . . . 132

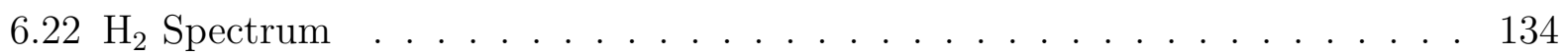

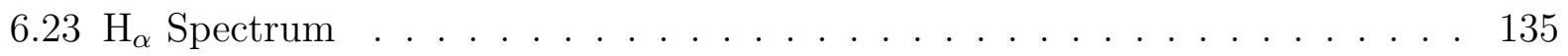

6.24 Axial Hydrogen Density . . . . . . . . . . . . . . . . . . 136

6.25 Mixed H-Kr Plasma . . . . . . . . . . . . . . . . . . . . . . . 137

7.1 Time resolved Kr density in source . . . . . . . . . . . . . . . . . . 143

7.2 Time resolved $\mathrm{Kr}$ density in expansion chamber . . . . . . . . . . . . . . 143

7.3 Time resolved $\mathrm{Kr}$ density vs power . . . . . . . . . . . . . . . . . . . 144

7.4 Neutral density vs. pulse length . . . . . . . . . . . . . . . . . 145

7.5 Time resolved $\mathrm{H}$ density in source . . . . . . . . . . . . . . . . 146

7.6 Time resolved radial $\mathrm{H}$ density . . . . . . . . . . . . . . . . . 146

7.7 Time resolved $\mathrm{H}$ temperature in source . . . . . . . . . . . . . . . 148

7.8 Time resolved radial $\mathrm{H}$ temperature . . . . . . . . . . . . . . . . . 148

7.9 Time resolved $\mathrm{H}$ density in source . . . . . . . . . . . . . . . . . 150

7.10 Wall loading . . . . . . . . . . . . . . . . . . . 151 
8.1 H-D resolution . . . . . . . . . . . . . . . . . . . . . 154

A.1 Pulsed triggering . . . . . . . . . . . . . . . . . . . . 158 


\section{Chapter 1}

\section{Introduction}

In fusion plasmas, neutrals act as fuel for the reaction, sputter impurities from the walls through collisions, and channel heat across magnetic field lines. Additionally, it is believed that neutrals play a key role in the transition between L (low) and H (high) tokamak confinement modes. $\mathrm{H}$ mode is widely accepted as the future ITER operating mode. Better measurement of neutrals in thermonuclear plasmas could greatly enhance understanding and control of the $\mathrm{L}$ to $\mathrm{H}$ transition. Low temperature processing plasmas rely on neutral uniformity for controlled etching and deposition. In processing applications, neutrals also form radicals, which can be detrimental. ${ }^{1}$ Better understanding of neutral dynamics could greatly improve the efficiency and selectivity of plasma processing systems, thereby reducing cost and increasing output. Therefore, the ability to perform absolute measurements of the neutral density profile is of critical importance for understanding and control of plasmas.

The purpose of this dissertation is two-fold. First is to outline and discuss the development of a new diagnostic to directly measure ground state neutral hydrogen and neutral krypton densities in plasmas. Neutral density measurements made in a helicon device using the new diagnostic provide confirmation that the diagnostic works. Second, through 
neutral density measurements in hydrogen and krypton plasmas, significant differences between noble gas and molecular gas plasmas are explored. While the work presented here was performed in a helicon source, the diagnostic was developed for use on a high temperature magnetic fusion device, specifically the DIII-D tokamak. Therefore, the role of neutral particles in tokamak plasmas and experimental considerations for installing the diagnostic on DIII-D is also reviewed in this work.

The remainder of this chapter is devoted to describing why neutral density measurements are important in plasma, even highly ionized plasma, experiments. The difficulties associated with making ground state measurements and a review of previous neutral density measurements are presented. Chapter 2 is devoted to a review of essential helicon physics. In Chapter 3, the experimental apparatus used in these experiments is described. Additional diagnostics used in these experiments are reviewed in Chapter 4. In Chapter 5, the development of a two-photon absorption laser induced fluorescence system, including considerations for installation on a fusion device, is presented. Neutral density measurements made in our helicon plasma source for three different fuel species: hydrogen, deuterium and krypton, are presented in Chapters 6 and 7. Both steady-state, Chapter 6, and time resolved, Chapter 7, measurements are reviewed and analyzed.

\subsection{Neutral density in helicon plasma sources}

In weakly ionized rf plasma sources, neutral particles play a major role in determining the plasma dynamics. Aside from their role of plasma fueling, collisions between plasma constituents and the much larger background of neutral particles affect many plasma phenomena. Specifically, ion-neutral collisions have been shown to affect plasma flows and ion beam for- 
mation in helicon sources. Charles et al. showed that neutral pressure has a major effect on the ion energy distribution function (IEDF). ${ }^{2}$ At low pressures, an energetic ion beam appears in the the IEDF. However, as pressure is increased the energetic beam diminishes. They theorized that ion-neutral charge exchange collisions suppressed the tail and could create a population of high energy, "hot", neutrals, though they had no direct measure of the neutrals. Such energetic neutrals could effect surface etching when helicons are used as plasma processing sources.

Sun et al., building on the work of Charles and Boswell ${ }^{3}$ and Cohen et al., ${ }^{4}$ demonstrated that current free double layers could only be formed for sufficiently low neutral pressures. ${ }^{5}$ They found an increase in the ion flow speed downstream of the antenna in an expanding magnetic field configuration with decreasing pressure. Below a certain pressure threshold, the flow became supersonic in the expansion region. They correlated the presence of the ion beam with the formation of a current free double layer. The strength of the double layer increased with decreasing pressure, probably a result of decreased collisionality.

Significant azimuthal rotation and shear flow have been observed in helicon sources. Holland et al. investigated this shear flow in an attempt to understand the physics of zonal flows in tokamaks. ${ }^{6}$ By assuming a uniform neutral density profile they found that the azimuthal ion velocity was insensitive to ion-neutral viscosity. Counter to this, Scime et al. found that ion-neutral viscosity had a major effect on azimuthal rotation and shear flow in a linear helicon device. ${ }^{7}$ They measured a decrease in azimuthal rotation with increasing fill pressure, see Figure 1.1. This suggests ion-neutral drag damped the rotation. Additionally, they found that the momentum exchange profile followed the neutral density profile. 

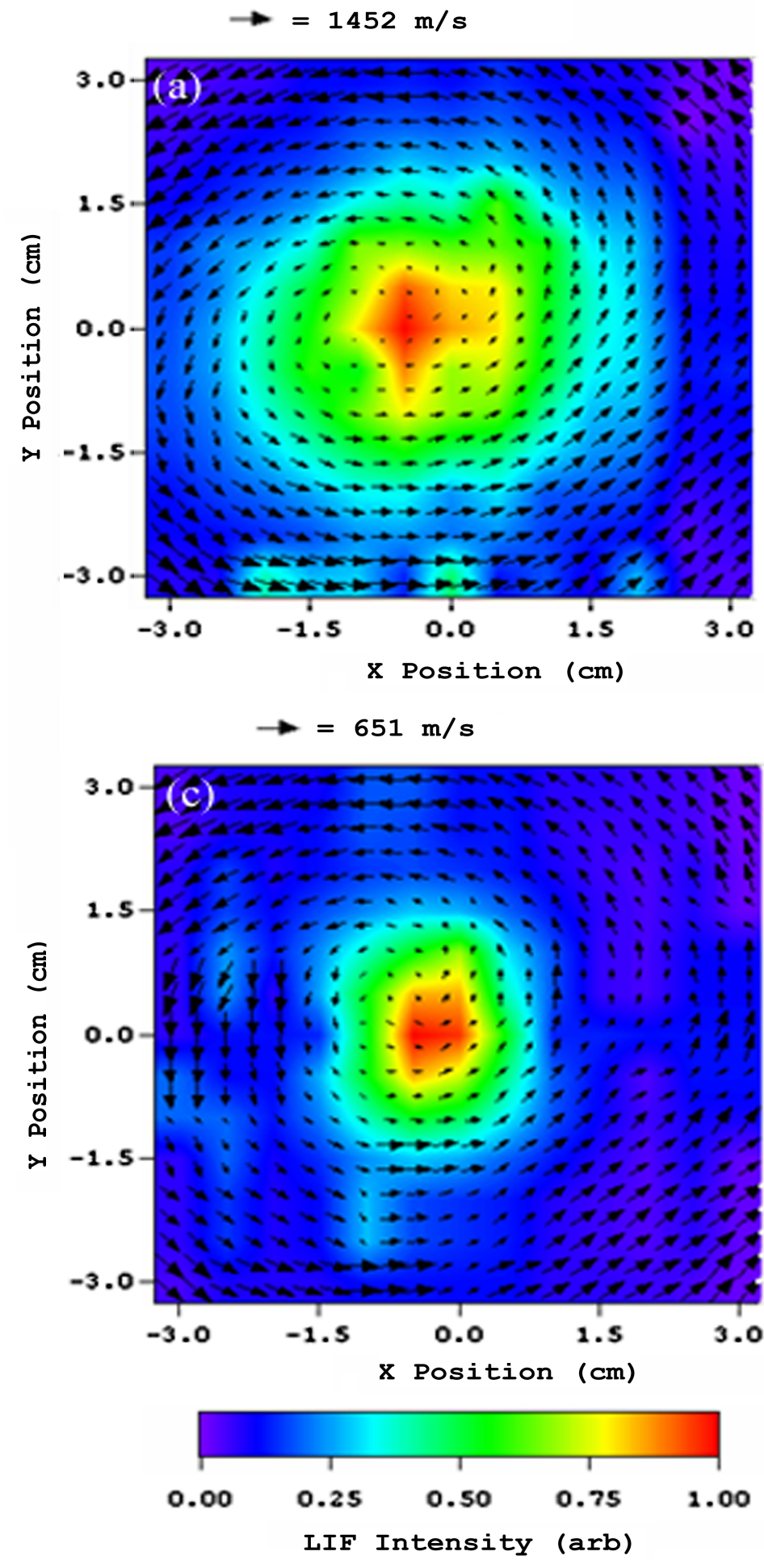

Figure 1.1: Azimuthal ion flows in a helicon plasma for low (top) and high (bottom) pressures, measured with excited state LIF. The largest rotation rates occurred at the lowest neutral pressures, $\sim 2.5 \mathrm{x}$ the high pressure rotation rate, suggesting the edge ion-neutral friction damped the rotation. ${ }^{7}$ 
In addition to influencing the dynamics of the plasma, neutrals have also been shown to exhibit significant dynamics themselves. Neutral depletion, a decrease in the on-axis neutral density beyond that expected due to ionization alone, has been observed and studied in a number of different experiments. Boswell first proposed $100 \%$ on-axis ionization in a helicon which he believed was consistent with pressure balance between the "hot" plasma in the core and "cold" gas in the edge, though he had no measure of the neutral density. ${ }^{8}$ Tynan measured 20-40\% neutral depletion in an argon helicon plasma, with the degree of depletion increasing with increasing rf power and fill pressure. ${ }^{9}$ These observations were in reasonable agreement with a simple 1-D model of neutral transport that assumed a uniform neutral temperature and small radial variation in neutral density. Similarly, Gilland et al. found neutral pressure (density) to decrease with increasing rf power, and hollow neutral profiles above a power threshold. ${ }^{10}$ Both Tynan and Gilland et al. utilized insertable capacitance manometers to measure neutral pressure and assumed uniform neutral temperature profiles to calculate the neutral density. Keesee and Scime calculated hollow neutral density profiles using excited state laser induced fluorescence and a collisional radiative model in an argon helicon plasma. ${ }^{11}$ They determined the degree of hollowness to vary significantly with source pressure. Using Doppler broadened LIF they were able to directly measure the neutral temperature. As neutrals typically only gain energy through collisions with ions, knowledge of the neutral temperature provides insight into ion-neutral energy and momentum exchange. ${ }^{12}$

While many neutral density measurements in helicon sources have been made using indirect measurement techniques, few direct measurements have been obtained. Using twophoton absorption laser induced fluorescence (TALIF), Aanesland et al. made direct measurements of ground state neutral density in a xenon helicon plasma. ${ }^{13}$ They found high, $\sim 85 \%$, on-axis, neutral depletion at their highest magnetic fields (150 G) and increasing de- 
pletion with source power. They measured a decrease in the edge pressure using a pressure gauge, but found an increase in edge neutral density for zero magnetic field. The neutral density increased with rf power for non-zero fields. They attributed the observed neutral depletion to pressure balance between the plasma and the neutrals. Their TALIF scheme was Doppler-free (described in Chapter 5), therefore they had no measure of the neutral temperature and therefore did not actually measure the neutral pressure.

The cause of enhanced neutral depletion, neutral pumping, is still poorly understood. However the mechanism has been described in two ways. Figure 1.2 depicts the first interpretation, where neutral particles (black) enter the plasma and are ionized by collisions with electrons (red). These fresh ions (blue) are then accelerated out of the plasma at the sound speed, $c_{s}=\sqrt{k_{B} T_{e} / m_{i}}$, to the plasma edge by a sheath electric field. In the low density edge, the ion recombines and then can diffuse back into the plasma as a neutral at the neutral thermal speed, $v_{t h}=\sqrt{k_{B} T_{n} / m_{n}}$, or be pumped away via a mechanical pump. The large discrepancy between the ion sound and neutral thermal speeds results in more neutrals transported to the edge than in the center, and a hollow neutral profile.

The second description is understood in terms of pressure balance and results from expulsion of neutrals from the plasma due to ion-neutral collisions. Because neutrals are the fuel for the plasma, creating a plasma necessarily decreases the neutral density. As plasma is preferentially created in the center this sets up an inward gradient in the plasma density and an outward gradient in the neutral density. When the plasma density and temperature become such that the plasma pressure becomes comparable to the neutral pressure, ion-neutral friction forces additional neutrals out of the core to maintain pressure balance, ${ }^{14}$ resulting in a hollow neutral profile. However, it is still not clear which of these mechanisms is responsible for neutral depletion, or if it is a combination of the two that results in the 


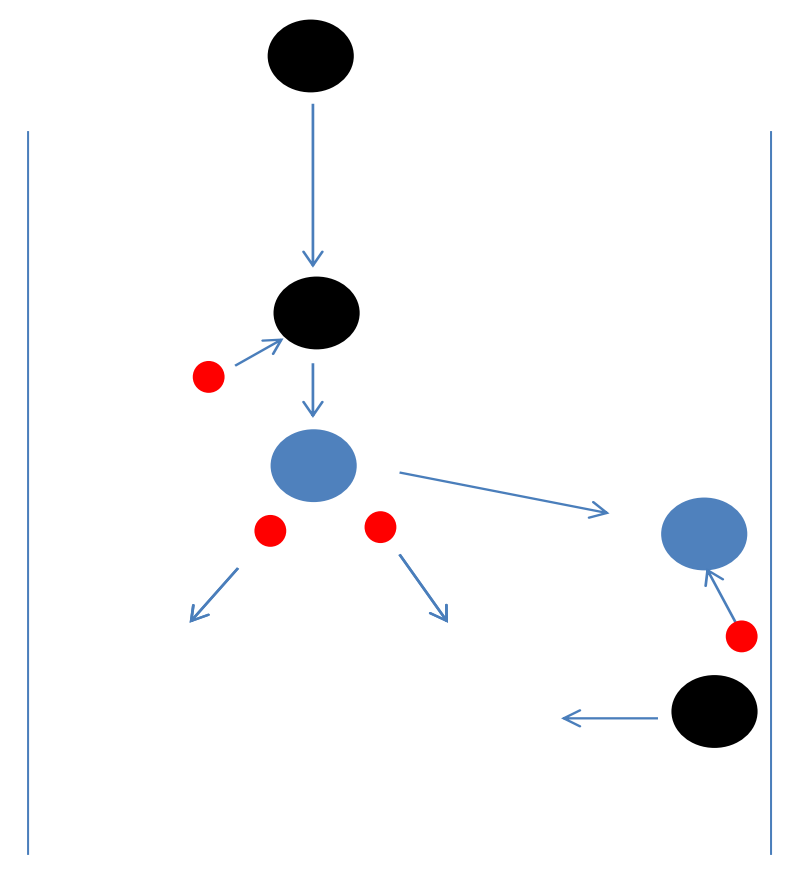

Figure 1.2: Cartoon depicting neutral pumping due to sheath electric field. Neutral particle (black), ion (blue), electron (red). See text for interpretation.

observed hollow profiles in helicon sources.

While a great deal of research has focused on monatomic helicon plasmas, only a small fraction of studies have employed molecular species. Molecular gas helicons are of considerable interest because of their relevance to both plasma processing and fusion applications. A discussion of hydrogen helicons is presented in Chapter 2.

While neutral depletion has been widely observed in noble gas helicons, few, if any, have reported it in molecular species. This could be due to the added sink for rf power that molecular dissociation presents. Both mechanisms for neutral depletion presented above require sufficient plasma densities for collisions to influence the plasma dynamics. However, as molecular dissociation can absorb power without creating plasma, lower plasma densities 
are typically produced in molecular species helicons. ${ }^{15}$ Thus, diffusion, not ionization, will determine the neutral particle dynamics. For example, in contrast to the significant neutral depletion measured in argon plasmas, Tynan et al. reported "highly uniform" atomic chlorine radial profiles in a helicon processing source, measured using an scannable optical probe. ${ }^{1}$ They also measured a small axial atomic chlorine density gradient, with the highest atomic density in the source region. This suggests that molecules are preferentially dissociated in the source region, where the plasma density is largest, then diffuse into the expansion region, creating a very uniform neutral density profile.

\subsection{Neutral density in fusion devices}

Neutral particles also play significant roles in near-fully ionized fusion devices. This is especially true in the low plasma density, low temperature edge of the plasma, see Figure 1.3 .

In addition to providing fuel for the plasma, as they do in rf sources, neutral-ion interactions, such as charge-exchange collisions, are a sink for momentum and energy. Neutralsurface interactions result in particle retention and wall loading. Lastly, neutrals are believed to play a major role in the $\mathrm{L}$ to $\mathrm{H}$ mode transition. It is important to note that these topics are inter-related. Charge-exchange affects $\mathrm{H}$ mode formation. $\mathrm{H}$ mode results in a change in the charge-exchange rate. Recycling due to particle retention leads to an increase in chargeexchange, increasing the $\mathrm{H}$ mode power threshold. During $\mathrm{H}$ mode, recycling decreases. Thus, while these effects are described individually they are strongly coupled. A comprehensive literature review of all neutral particle measurements in fusion devices is beyond the scope of this dissertation. Only selected sources, and references contained therein, are 


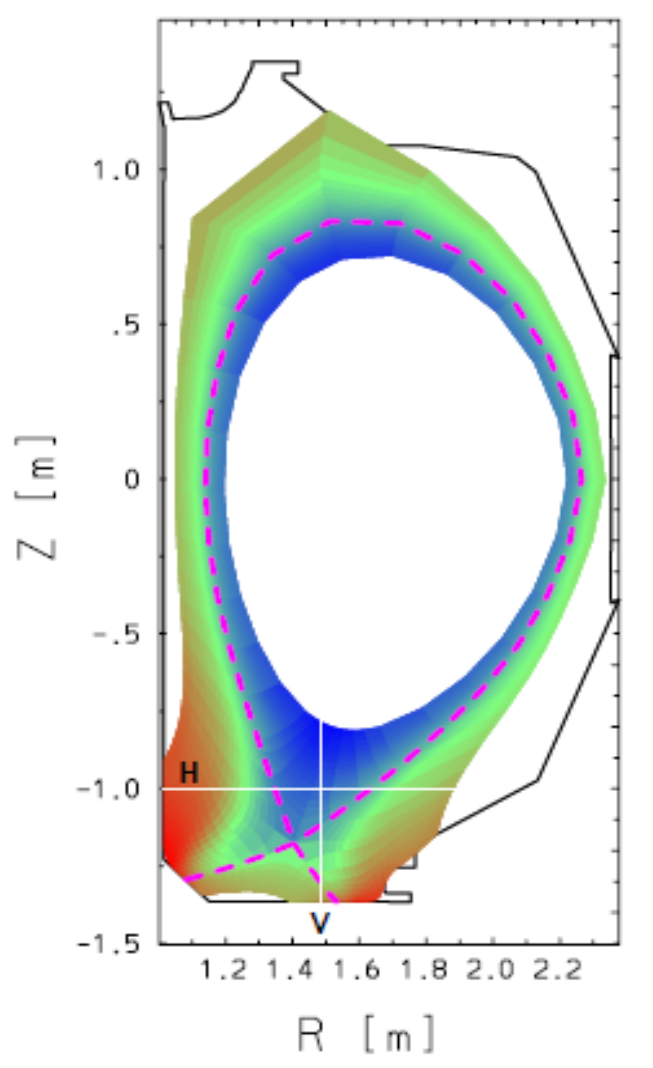

Figure 1.3: Edge region of the DIII-D tokamak. The dashed red line is the last closed flux surface, or separatrix. Outside of the separatrix the neutral density increases by $\sim 4$ orders of magnitude, from $10^{15} \mathrm{~m}^{-3}$ to $10^{19} \mathrm{~m}^{-3}$. Color represents modeled neutral density.

presented to highlight these issues.

\subsubsection{Charge-Exchange}

The dominant neutral-plasma interaction in a fusion plasma is through charge-exchange collisions between neutrals and ions.

$$
H+H^{+} \rightarrow H^{+}+H
$$


The reaction rate for charge-exchange is larger than any other mechanism at almost any temperature, as shown in Figure 1.4. Thus, through charge-exchange, neutrals play a critical role in determining the dynamics of tokamak plasmas.

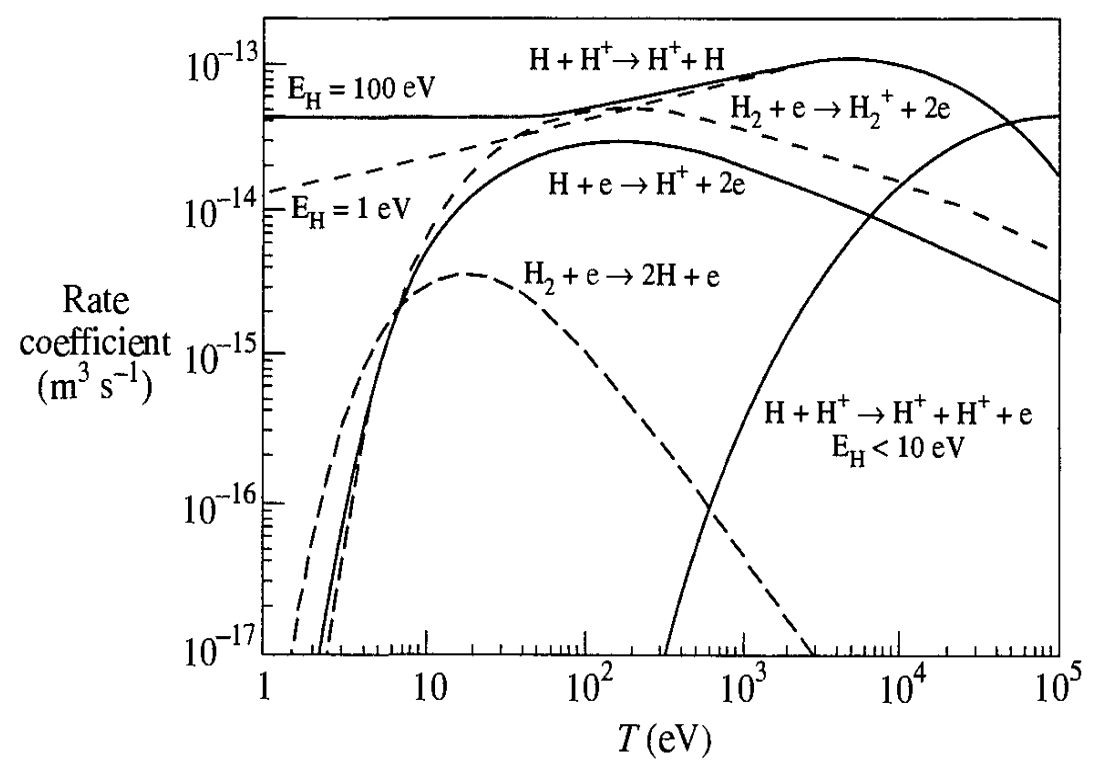

Figure 1.4: Rate coefficients for various neutral hydrogen processes. Charge-exchange has the largest rate coefficient for energies $<5 \times 10^{4} \mathrm{eV} .{ }^{16}$

Charge-exchange collisions can be thought of as elastic neutral scattering, with the ions and neutrals exchanging identities. ${ }^{17}$ Even though momentum is not directly lost through the collision, bulk plasma momentum can be lost as "hot" charge-exchanged neutrals, not bound by magnetic field lines, leave the plasma, and thereby lower the overall momentum content. ${ }^{18}$ Momentum loss has a number of deleterious effects including increased neutral penetration, and slowing of plasma rotation.

Neutral particles moving from the low temperature edge of the plasma into the high 
temperature core region is known as neutral penetration. The distance these neutral particles travel before they are ionized is the neutral penetration length. Once the cold edge neutrals cross into the hotter core they charge-exchange with hot core ions. The fresh hot neutrals then carry heat and momentum out across the separatrix, the last closed flux surface, increasing radial transport of heat flux, particle flow and power loss. ${ }^{19}$ The increase in power loss affects plasma parameters in the pedestal region, which then cause changes in the confinement of the core plasma. The neutral penetration length due to ionization is

$$
\lambda_{n} \sim \frac{v_{n}}{n_{e}<\sigma v>_{i o n}}
$$

where $v_{n}$ is the neutral velocity, $n_{e}$ is the electron density, and $\langle\sigma v\rangle_{\text {ion }}$ is the ionization rate. $^{20}$ If we assume edge neutrals are purely the result of wall recycling, and therefore are roughly room temperature, and let the edge electron density and temperature to be $\sim 10^{19} \mathrm{~m}^{-3}$ and $10 \mathrm{eV}$, respectively, the neutral penetration length due purely to ionization is $<5 \mathrm{~cm}$ (see Figure 1.5).

As charge-exchange results in hot ions and neutrals exchanging identities, the resulting hot neutrals can penetrate further before re-ionization. A $10 \mathrm{eV}$ neutral has a penetration length due to charge-exchange of $\sim 30 \mathrm{~cm}$, as shown in Figure 1.5. A neutral undergoing a number of such collisions will penetrate further into the plasma, until it is ultimately ionized. This allows neutrals to penetrate into the core of the plasma despite temperatures above the ionization potential. ${ }^{19}$

Tokamak plasmas inherently rotate, both toroidally and poloidally. Initially, it was believed that this rotation was the result of parallel momentum input from neutral beam injection (NBI). ${ }^{16}$ However, plasma rotation has been observed in the absence of direct mo- 


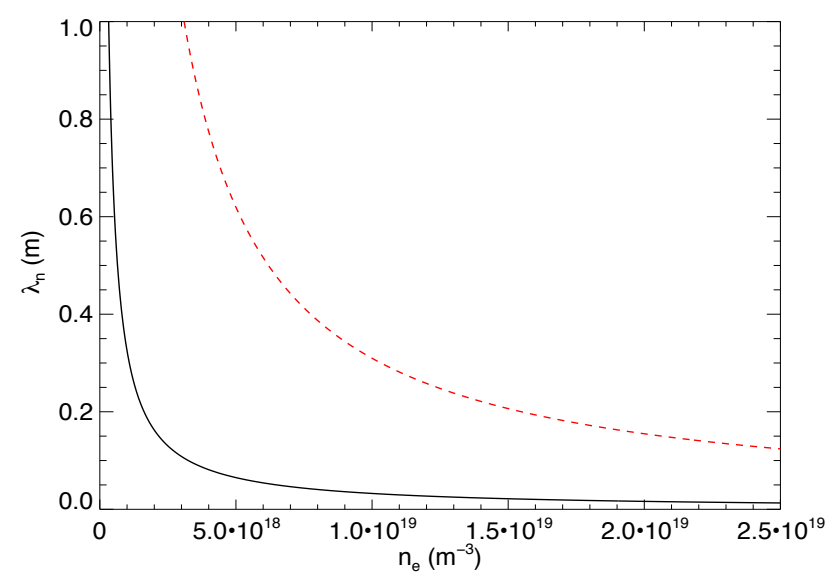

Figure 1.5: $\mathrm{H}$ ionization length for room temperature neutrals (solid black) and charge-exchange length for $10 \mathrm{eV}$ neutrals (dashed red).

mentum input, in both $\mathrm{rf}^{21}$ and Ohmically ${ }^{22}$ heated tokamaks, and is present in both L and $\mathrm{H}$ modes. ${ }^{23}$ This rotation is advantageous, as it suppresses instabilities such as resistive wall modes and neoclassical tearing modes. ${ }^{23}$ However, typical velocity profiles show the rotation decreases in the plasma edge (see Figure 1.6) indicating some drag force at the boundary. ${ }^{16}$

The most likely cause of this drag force is ion-neutral friction resulting from chargeexchange collisions. Rowan et al. investigated the effect of momentum loss through chargeexchange on the Texas Experimental Tokamak (TEXT). ${ }^{18}$ Using Doppler shifted impurity emission lines they determined that charge-exchange damped toroidal rotation, but had no discernible affect on poloidal rotation. Additionally, they found that in order for neoclassical theory to predict the magnitude of rotation, momentum loss due to charge-exchange had to be included.

In addition to suppressing instabilities, rotation establishes a radial electric field, which is believed to play a role in the $\mathrm{H}$ mode transition. During L mode the magnitude of the electric 


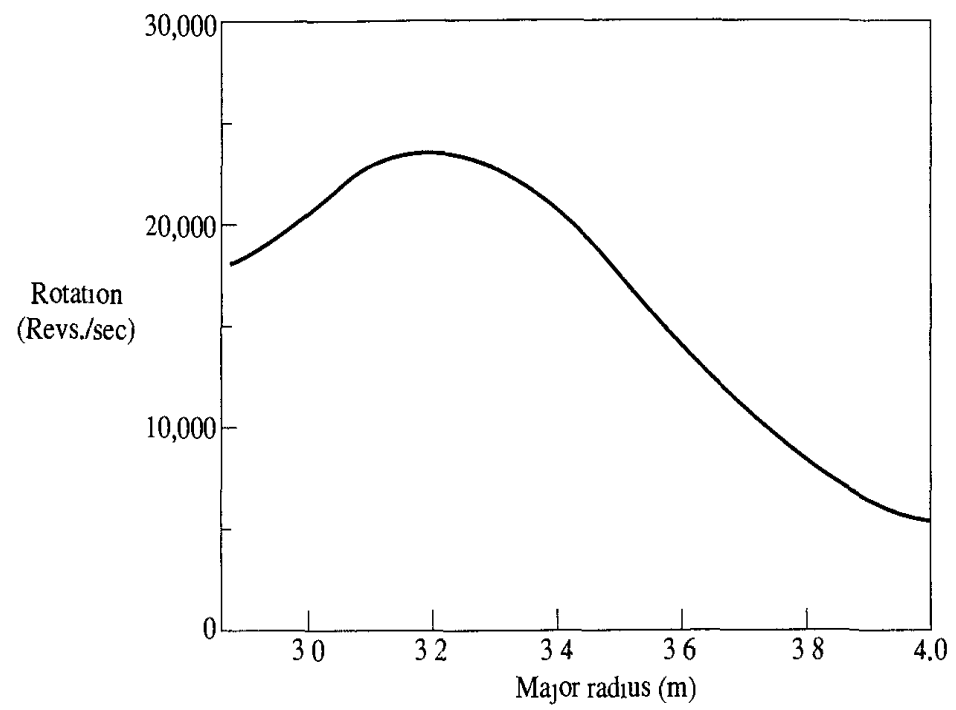

Figure 1.6: Rotation rate profile in the JET tokamak. The decrease in the edge rotation rate suggests a drag force, most likely from ion-neutral charge-exchange collisions resulting in momentum loss. ${ }^{16}$

field is small. In $\mathrm{H}$ mode, the electric field changes direction, and increases in magnitude. The cause for the field reversal is, at present, unknown. Neutral friction due to charge exchange reduces the electric field by damping toroidal rotation, affecting the transition between $\mathrm{L}$ and $\mathrm{H}$ modes. ${ }^{24}$

\subsubsection{H Mode}

The thermal energy content of a plasma is

$$
W=\int 3 n T d^{3} x=3<n T>V
$$


where $n, T$, and $V$ are the plasma density, temperature, and volume, respectively. From this, the rate of energy loss of the plasma is defined as

$$
P_{L}=\frac{W}{\tau_{E}},
$$

where $\tau_{E}$ is the energy confinement time. Energy loss is balanced by an external heating source $P_{H}$ such that

$$
P_{L}=P_{H}
$$

The energy confinement time is then determined from experimentally measured values,

$$
\tau_{E}=\frac{W}{P_{H}}
$$

To achieve sustained thermonuclear fusion, $\tau_{E}$ must be made sufficiently long such that the plasma is heated by the $3.5 \mathrm{MeV} \alpha$-particles resulting from the fusion reaction.

The baseline energy confinement mode in a tokamak is known as the low confinement mode (L mode). It is characterized by "poor" energy confinement time, which degrades with increasing power. ${ }^{25}$ Under the right conditions, with additional heating power, an abrupt, and initially unexpected, change to the high confinement mode ( $\mathrm{H}$ mode) occurs. The transition was first observed in the ASDEX tokamak in $1982^{26}$ and typically results in a 2-3x increase in the energy confinement time, see Figure 1.7

In addition to an increase in energy confinement time, the particle confinement time increases during $\mathrm{H}$ mode. This results in an increase in temperature and edge plasma density. The increase in edge plasma density flattens the inner density profile, normally centrally peaked, and steepens the edge density gradient (see Figure 1.8.) 


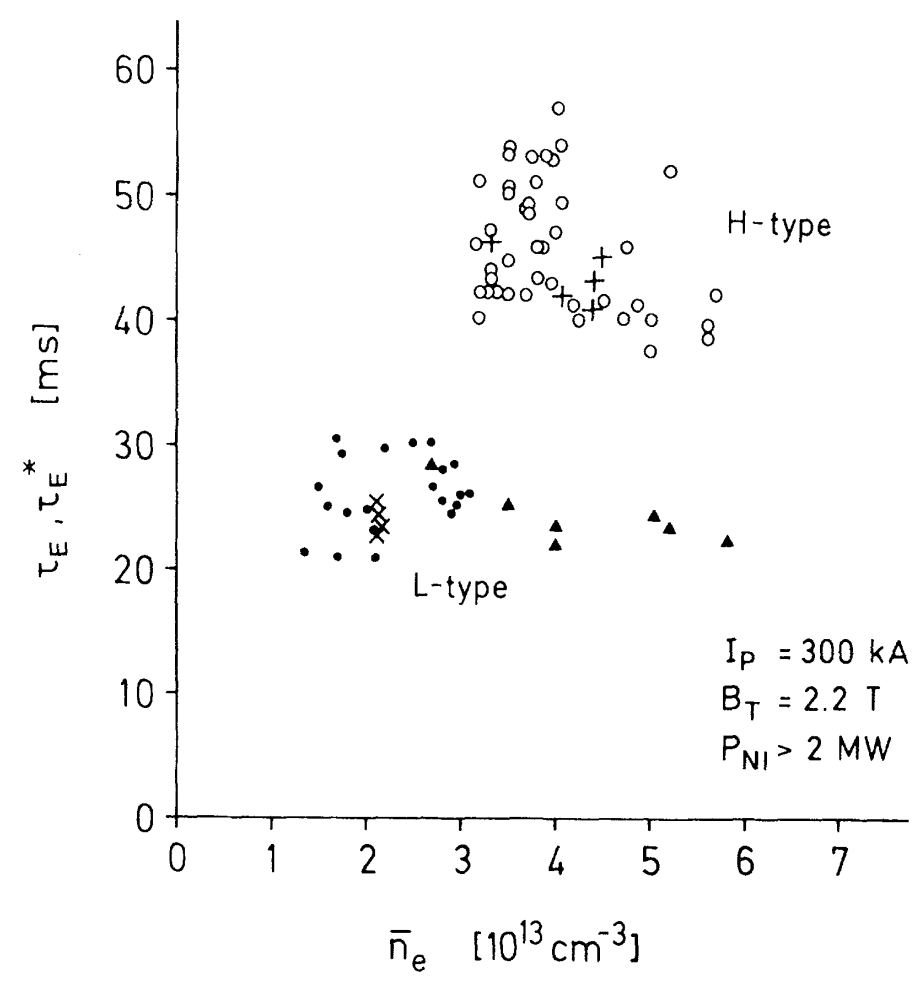

Figure 1.7: Energy confinement time is ASDEX tokamak during L and H modes. ${ }^{26}$

The increase in the edge density gradient indicates formation of a transport barrier holding the plasma in. To understand the process, consider a sandpile. Under normal circumstances, sand added to the edge of the pile will fall off, and the profile of the pile remains centrally peaked. If some barrier is constructed around the edge of the pile, the added sand will be held in and the core profile flattens. While this is not exactly what is occurring in the tokamak edge, it provides a mental model of the physical process.

Initially, $\mathrm{H}$ mode was only achievable with auxiliary heating from neutral beam injection (NBI). However, $\mathrm{H}$ mode is now routinely achieved with other auxiliary heating mechanisms, such as rf waves, ${ }^{28}$ or even in purely Ohmic discharges ${ }^{22}$ without any auxiliary heating.

Numerous theories have been proposed as the physical mechanism that results in the L-H transition. Entire dissertations could be devoted to explaining these theories. It has 


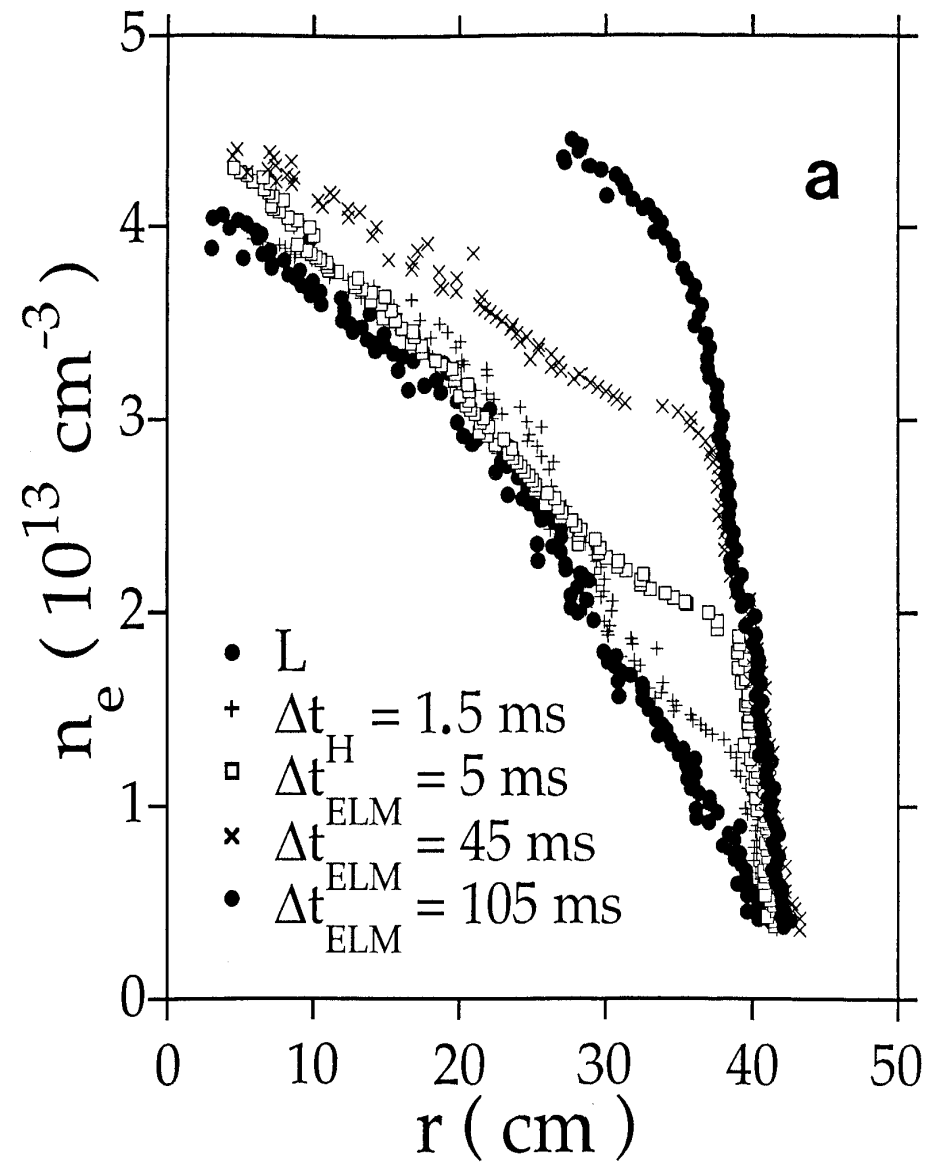

Figure 1.8: Plasma density versus radius for five different times in the ASDEX tokamak following the L-H transition. Note the increasing height of the density pedestal with increasing time. ${ }^{27}$

been proposed that charge-exchange friction damps the poloidal component of the $E \times B$ shear flow, which affects the transition power threshold. By combining $D_{\alpha}$ emission data and a theoretical neutral transport model, Carreras et al. calculated the poloidal neutral density distribution. ${ }^{29}$ Using this, they determined that the power threshold in DIII-D scaled with the maximum charge-exchange frequency on a fixed flux surface. This allowed them to quantify the effect of neutral particles on the L-H transition and construct a scaling law for the power threshold including neutrals, something that had not been done prior.

Neutral hydrogen in the edge results from recycling, the process where an ion encounters 
a material surface, is neutralized and then released back into the plasma. ${ }^{16}$ Associated with the L-H transition is a characteristic drop in the $H_{\alpha}\left(D_{\alpha}\right)$ emission signal, see Figure 1.9.

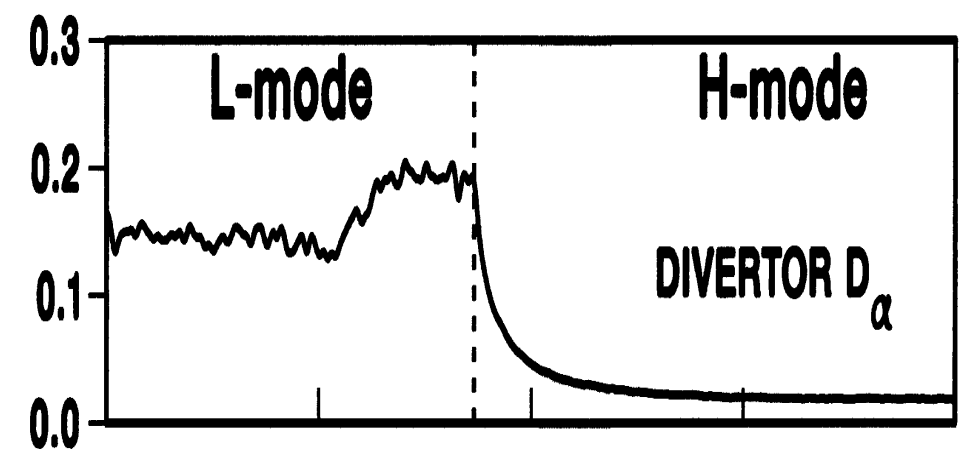

Figure 1.9: $\mathrm{D}_{\alpha}$ signal during $\mathrm{L}$ to $\mathrm{H}$ transition. Horizontal axis is discharge time. ${ }^{27}$

The decrease in the $H_{\alpha}$ signal during $\mathrm{H}$ mode has been correlated with a decrease in recycling. Recent work on the Experimental Advanced Superconducting Tokamak (EAST) has also demonstrated the role edge neutrals play in the power threshold for the L-H transition. $X u$ et al. found that $\mathrm{H}$ mode in EAST could only be achieved after extensive wall conditioning reduced the edge neutral density. ${ }^{30}$ Many tokamaks undergo wall conditioning in an attempt to reduce recycling. Some type of hydrogen getter, a material that bonds to or adsorbs hydrogen is applied to the walls. The getter material traps impinging ions/neutrals, preventing their release back into the plasma. EAST applies lithium, both before and during discharges, to reduce recycling. With extensive application of lithium, the recycled edge neutral density was reduced and $\mathrm{H}$ mode was achieved. The results from EAST suggest that the decrease in recycling leads to $\mathrm{H}$ mode. However, it has also been shown that $\mathrm{H}$ mode itself reduces recycling by creating a transport barrier that prevents plasma impinging on the walls. Ultimately, which is the cause, and which is the effect is currently unknown.

Another theory for $\mathrm{H}$ mode formation is an influx of neutrals leading to an increase in 
plasma density inside the separatrix. The local increase in plasma density creates a transport barrier that suppresses turbulence and increases energy confinement. ${ }^{31}$ Using $L y_{\alpha}$ emission, Boivin et al. measured the ionization rate inside and outside the separatrix in L and $\mathrm{H}$ modes in the Alcator C-Mod tokamak. ${ }^{19}$ They found the ionization rate peaks near the separatrix in L mode, consistent with a 1-D Monte Carlo neutral transport simulation. However, in $\mathrm{H}$ mode, the ionization rate peaked inside the separatrix, consistent with the development of a density pedestal leading to a transport barrier during $\mathrm{H}$ mode. ${ }^{32}$ It has been shown that the barrier width is well correlated with the neutral penetration length. ${ }^{33,34}$ Whether the transport barrier suppresses turbulence and leads to $\mathrm{H}$ mode, or $\mathrm{H}$ mode causes the transport barrier is still unknown.

While $\mathrm{H}$ mode is now routinely achieved in almost all tokamaks worldwide and a few stellerators, the trigger for the mechanism remains a mystery. It is widely believed, as described above, that neutral particles, specifically edge neutrals, play a major role in the L to $\mathrm{H}$ transition. Due to lack of direct measurements of neutrals during the transition their true role remains ambiguous and they are considered a "hidden" variable in the transition mechanism. ${ }^{35}$

\subsubsection{Neutral Retention/Recycling}

The impact of neutral retention and recycling on a tokamak plasma cannot be overstated. As was described in the previous section, when an ion encounters a material surface, it can be neutralized and embed itself in the surface. Trapped neutrals are then released in subsequent discharges due to particle flux to the walls. These "dynamically" released neutrals can influence energy confinement and make density control impossible, ${ }^{36}$ which ultimately leads to density limit-based disruptions. In large machines, such as JET and ITER, such 
disruptions could destroy the vacuum vessel ${ }^{37}$ and thus must be avoided. Additionally, in a burning plasma experiment, tritium retention in first wall materials will affect fuel cycles and must be kept below the levels allowed by site licensing. ${ }^{38}$

One of the major questions for future fusion devices is what to use as a first wall material. Early tokamaks relied on stainless steel first walls, however it was soon shown that stainless steel rapidly eroded due to sputtering. The sputtered stainless steel contaminated the plasma, leading to increases in impurity radiation, thereby lowering the plasma energy content. Many tokamaks have upgraded to carbon first walls, and, as previously described, undergo extensive wall coating to reduce the deleterious effects of sputtering and recycling. The proposed first wall for ITER is a combination of beryllium, carbon and tungsten tiles. ${ }^{39}$ High temperature tungsten develops what is known as "fuzz", which is a $\mu \mathrm{m}$ scale surface imperfection. ${ }^{40}$ Little is known regarding how this fuzz will affect recycling and neutral retention.

\subsection{Neutral density measurement techniques}

A number of techniques exist to measure neutral gas density. As with all diagnostics, these can be broken down into two categories: perturbative and non-perturbing. Ideally, all diagnostics would be non-perturbing as this allows measuring plasma properties without directly interacting with the plasma. However, in some circumstances achieving such a condition is impossible and perturbing diagnostics must be incorporated.

The simplest method to measure density is with a pressure gauge. Pressure gauges present a number of issues that make them non-ideal as density diagnostics. They are an indirect measure of density. Pressure is the product of density and temperature and without 
accurate knowledge of the neutral temperature an accurate density measurement cannot be made with a pressure gauge. These gauges are typically mounted on the chamber wall, providing only edge measurements. To make an internal measurement the gauge must be inserted into the plasma, which is accomplished by coupling the gauge to some sort of an insertable probe. ${ }^{10}$ Mounted on a probe the measurement becomes perturbing. Lastly, the majority of pressure gauges do not differentiate between species. Resolving the pressures of neutral constituents of a multi-species plasma is therefore impossible.

Electron-ion and electron-neutral collisions in a plasma result in a distribution of excited states. Spontaneous decay from excited to low energy states results in radiative emission. This natural emission can reveal a great deal of information about the plasma. However, because the emission is the result of decay from an excited state, the information gained is about that excited state. A model, either coronal or collisional radiative, is required to determine ground state quantities from the excited state emission signal. These models require a number of assumptions regarding excited state distributions and are typically very difficult to construct, except for the simplest of species. Even for the simplest of species, the various process rate coefficients (excitation, ionization, recombination, etc.) are very sensitive to plasma temperature, especially for $T_{e}<10 \mathrm{eV}$. Thus, highly accurate plasma temperature measurements are necessary for such models to produce accurate measures of the neutral density.

Emission spectroscopy overcomes many of the issues associated with pressure gauges. Edge mounted collection optics look into the interior of the plasma, a region edge mounted pressure gauges cannot directly measure. However, these edge emission measurements are line-integrated. Achieving localized measurements requires multiple viewing angles and a profile reconstruction technique, such as Abel inversion. ${ }^{41}$ A number of groups have at- 
tempted to make localized emission measurements with collection optics mounted on probes inserted in the plasma. ${ }^{1}$ However, as was already described with pressure gauges, this makes the measurement perturbing, eliminating one of the major advantages of spectroscopy.

In addition to the necessity of using a model to determine ground state quantities, emission measurements typically suffer from poor spectral resolution. This is due to the need to use a dispersing element to separate spectral components. As an example, consider a mixed hydrogen and deuterium plasma, as would be found in a fusion device. The $H_{\alpha}$ and $D_{\alpha}$ emission lines are separated by $\sim 0.2 \mathrm{~nm}(100 \mathrm{GHz})$, and overlap significantly for temperatures above $10 \mathrm{eV}$, see Figure 1.10.

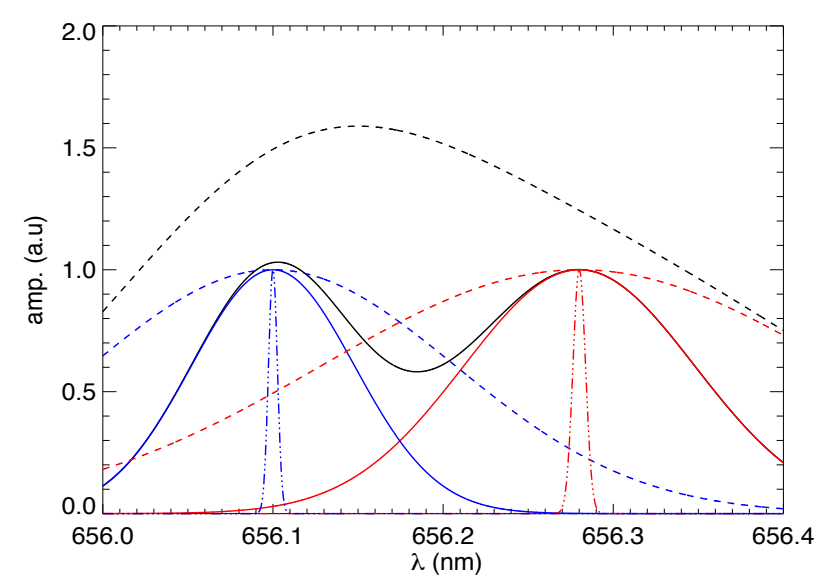

Figure 1.10: $H_{\alpha}$ (red)- $D_{\alpha}$ (blue) splitting for room temperature (dot-dashed), $10 \mathrm{eV}$ (solid) and $50 \mathrm{eV}$ (dashed) The lines overlap significantly at $10 \mathrm{eV}$, and are indistinguishable at $50 \mathrm{eV}$.

Resolving flows, however requires precision that is nearly unachievable with emission spectroscopy. To resolve a flow of $1 \mathrm{~km} / \mathrm{s}$ at $656 \mathrm{~nm}$ requires a resolution of better than $1 \mathrm{GHz}$. A typical spectrometer with a groove density of 1200 groove/mm and slit width of $1 \mu \mathrm{m}$ would need to be $\sim 10 \mathrm{~m}$ long to have this type of resolution. A single mode laser, used for 
laser induced fluorescence, has a bandwidth of better than $100 \mathrm{MHz}$, typically in the 10s of $\mathrm{MHz}$, and thus, can easily resolve flows and overlapping absorption lines with much higher precision.

Absorption spectroscopy, specifically laser induced fluorescence (LIF), solves the localization and spectral resolution issues associated with emission spectroscopy. LIF is a well established technique for measuring neutral and ion properties. ${ }^{42,43}$ Absorption of an input photon results in an upward transition of a bound electron from a low to high energy state. The electron then spontaneously decays, emitting a photon of energy equal to the energy difference between the excited and final energy states. As shown in Figure 1.11, the decay can be back to the original state, or to a third, different energy state.
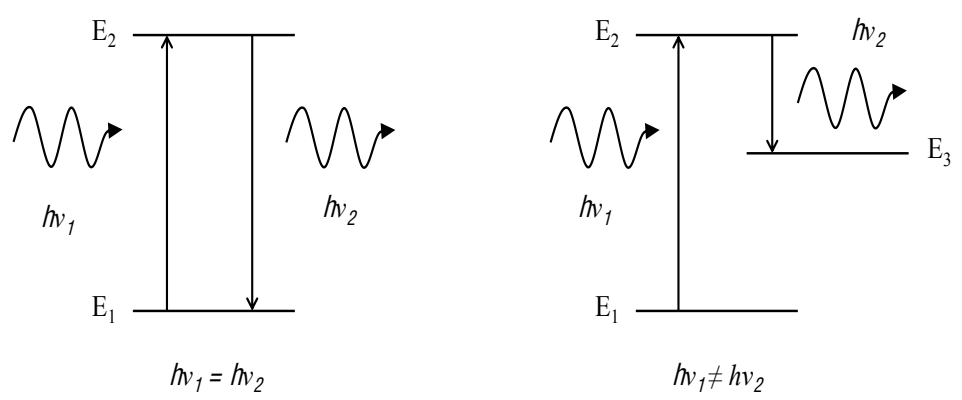

Figure 1.11: Basic LIF mechanism. Two-state schemes are known as resonant fluorescence (left), the absorbed and emitted photons are of equal wavelength. Three state schemes are non-resonant (right) and the absorbed and emitted photon are of unequal wavelengths.

Because the upward transition is induced by the input photon, the information gained by recording the subsequent decay is about the absorbing, rather than emitting, state. LIF is a very localized technique, with the emission only coming from the location of the laser beam, rather than the plasma as a whole, as is the case with emission spectroscopy. This permits investigation of a spatially localized region of the plasma. As previously noted, single 
mode lasers that are employed in LIF studies typically have bandwidths of a few MHz, much narrower than conventional spectrometers. Temperatures of $0.1 \mathrm{~s} \mathrm{eV}$ and flows of $100 \mathrm{~s} \mathrm{~m} / \mathrm{s}$ are easily resolved with these lasers.

A drawback of LIF is the need for a large absorbing population to achieve measurable signal levels. This need is satisfied by pumping transitions from the ground or metastable (long lived) states. In hydrogen, two schemes, shown in Figure 1.12, are typically used, the $H_{\alpha}$ transition at $656 \mathrm{~nm}$, and the $L y_{\alpha}$ transition at $121 \mathrm{~nm}$.
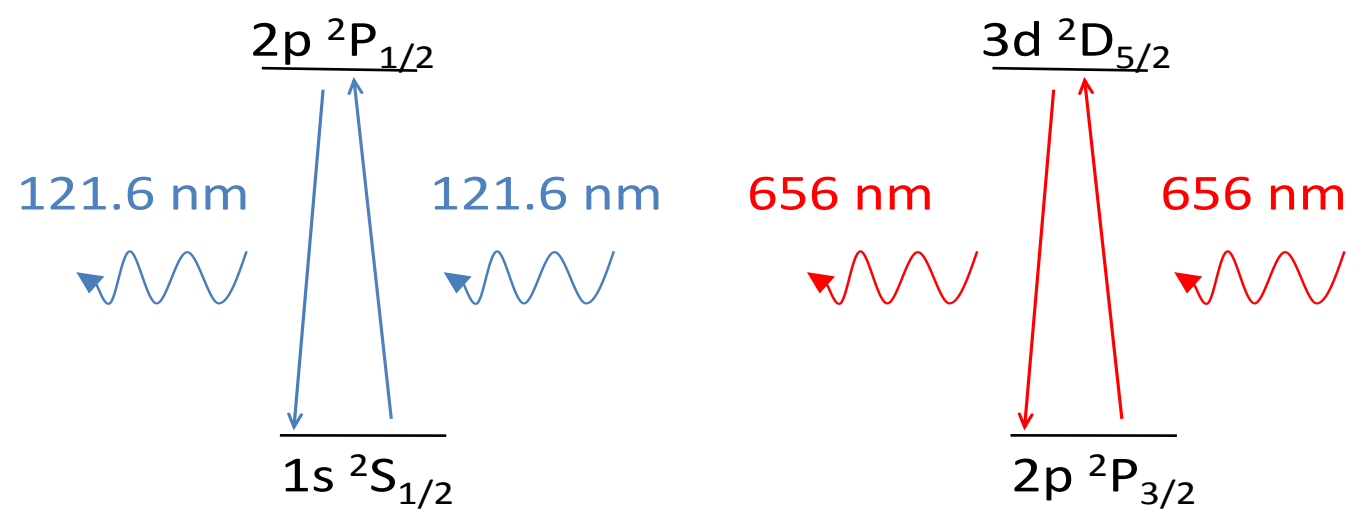

Figure 1.12: Two possible LIF schemes for neutral hydrogen, $L y_{\alpha}$ (left) and $H_{\alpha}$ (right).

Both of these schemes present challenges. Both are resonant fluorescence schemes. That is, both have absorption and emission occurring at the same wavelength. The indistinguishability of the incident and fluorescent emission causes scattered laser light issues that cannot be overcome with spectral filtering and lock-in amplification, as is typically done with non-resonant LIF schemes. The $H_{\alpha}$ scheme is an excited state scheme. Thus, as with emission spectroscopy, a model is required to determine ground state quantities from the measured signal. The $L y_{\alpha}$ scheme is a ground state scheme. However, both the absorption and emission wavelengths are in the vacuum ultraviolet (VUV), which makes light injection 
and collection problematic due to the paucity of VUV transparent materials.

Due to the number of issues associated with each of the diagnostics described above, a new, highly localized, non-perturbing, direct ground state measurement technique is required. TALIF is such a technique. The measurement is non-perturbing and highly localized. Additionally, because two photons are used to supply the transition energy, higher energy ground state transitions not achievable with single photon absorption are directly accessible. A complete discussion of TALIF, including the TALIF system designed and constructed at WVU is presented in Chapter 5 . 


\section{References}

[1] G. R. Tynan, A. D. Bailey, G. A. Campbel, R. Charatan, A. de Chambrier, G. Gibson, D. J. Hemker, K. Jones, A. Kuthi, T. S. C. Lee, and M. Wilcoxson, "Characterization of an azimuthally symmetric helicon wave high density plasma source," Journal of Vacuum Science 8 Technology, A: Vacuum, Surfaces, and Films, vol. 15, pp. 2885-2892, 1997.

[2] C. Charles, R. W. Boswell, and R. K. Porteous, "Measurement and modeling of ion energy distribution functions in a low pressure argon plasma diffusing from a $13.56 \mathrm{mhz}$ helicon source," Journal of Vacuum Science \& Technology, A: Vacuum, Surfaces, and Films, vol. 20, pp. 398-403, 1992.

[3] C. Charles and R. Boswell, "Current-free double-layer formation in a high-density helicon discharge," Applied Physics Letters, vol. 82, pp. 1356-1358, 2003.

[4] S. A. Cohen, N. S. Siefert, S. Strange, R. F. Boivin, E. E. Scime, and F. M. Levinton, "Ion acceleration in plasmas emerging from a helicon-heated magnetic-mirror device," Physics of Plasmas, vol. 10, pp. 2593-2598, 2003.

[5] X. Sun, R. H. C. Biloiu, and E. E. Scime, "Parallel velocity and temperature of argon ions in an expanding, helicon source driven plasma," Plasma Sources Science and Technology, vol. 13, pp. 359-370, 2004.

[6] C. Holland, J. H. Yu, A. James, D. Nishijima, M. Shimada, N. Taheri, and G. R. Tynan, "Observation of turbulent-driven shear flow in a cylindrical laboratory plasma device," Physical Review Letters, vol. 96, p. 195002, 2006.

[7] E. Scime, R. Hardin, C. Biloiu, A. M. Keesee, and X. Sun, "Flow, flow shear, and related profiles in helicon plasmas," Physics of Plasmas, vol. 14, p. 043505, 2007. 
[8] R. W. Boswell, "Very efficient plasma generation by whistler waves near the lower hybrid frequency," Plasma Physics and Controlled Fusion, vol. 26, pp. 1147-1162, 1984.

[9] G. R. Tynan, "Neutral depletion and transport meachanisms in large-area high density plasma sources," Journal of Applied Physics, vol. 86, p. 5356, 1999.

[10] J. Gilland, R. Breun, and N. Hershkowitz, "Neutral pumping in a helicon discharge," Plasma Sources Science and Technology, vol. 7, pp. 416-422, 1998.

[11] A. M. Keesee and E. E. Scime, "Neutral density profiles in argon helicon plasmas," Plasma Sources Science and Technology, vol. 16, p. 742, 2007.

[12] F. Anderegg, R. A. Stern, F. Skiff, B. A. Hammel, M. Q. Tran, P. J. Paris, and P. Kohler, "Ion heating due to rotation and collision in magnetized plasma," Physical Review Letters, vol. 57, pp. 329-332, 1986.

[13] A. Aanesland, L. Liard, G. Leray, J. Jolly, and P. Chabert, "Direct measurements of neutral density depletion by two-photon absorption laser-induced fluorescence spectroscopy," Applied Physics Letters, vol. 91, p. 121502, 2007.

[14] A. Fruchtman, G. Makrinich, P. Chabert, and J. M. Rax, "Enhanced plasma transport due to neutral depletion," Physical Review Letters, vol. 95, p. 115002, 2005.

[15] Y. Sakawa, T. Takino, and T. Shoji, "Contribution of slow waves on production of high-density plasmas by $\mathrm{m}=0$ helicon waves," Physics of Plasmas, vol. 6, pp. 47594766, 1999.

[16] J. Wesson, Tokamaks. Clarendon Press, 2004. 
[17] R. D. Hazeltine, M. D. Calvin, P. M. Valanju, and E. R. Solano, "Analytical calculations of neutral transport and its effect on ions," Nuclear Fusion, vol. 32, p. 3, 1992.

[18] W. L. Rowan, A. G. Meigs, E. R. Solano, P. M. Valanju, M. D. Calvin, and R. D. Hazeltine, "Rotation in ohmically heated tokamaks: Experiment and theory," Physics of Fluids B, vol. 5, pp. 2485-2490, 1993.

[19] R. L. Boivin, J. A. Goetz, A. E. Hubbard, J. W. Hughes, I. H. Hutchinson, J. H. Irby, B. LaBombard, E. S. Marmar, D. Mossessian, C. S. Pitcher, J. L. Terry, B. A. Carreras, and L. W. Owen, "Effects of neutral particles on edge dynamics in alcator c-mod plasmas," Physics of Plasmas, vol. 7, pp. 1919-1926, 2000.

[20] R. J. Goldston and P. H. Rutherford, Introduction to Plasma Physics. IOP Publishing, 1995.

[21] J. E. Rice, M. Greenwald, I. H. Hutchinson, E. S. Marmar, Y. Takase, S. M. Wolfe, and F. Bombarda, "Observations of central toroidal rotation in icrf heated alcator c-mod plasmas," Nuclear Fusion, vol. 38, pp. 75-85, 1998.

[22] I. H. Hutchinson, J. E. Rice, R. S. Granetz, and J. A. Snipes, "Self-acceleration of a tokamak plasma during ohmic h mode," Physical Review Letters, vol. 84, pp. 3330-3333, 2000.

[23] A. Scarabosio, A. Bortolon, B. P. Duval, A. Karpushov, and A. Pochelon, "Toroidal plasma rotation in the tcv tokamak," Plasma Physics and Controlled Fusion, vol. 48, pp. 663-683, 2006.

[24] A. G. Peeters, "Equations for the evolution of the radial electric field and poloidal 
rotation in toroidally symmetric geometry," Physics of Plasmas, vol. 5, pp. 763-767, 1998.

[25] S. I. Itoh, K. Itoh, and A. Fukuyama, "Physics of edge plasma in enhanced confinement modes," Journal of Nuclear Materials, vol. 220-222, pp. 117-131, 1995.

[26] F. Wagner, G. Becker, K. Behringer, D. Campbell, A. Eberhagen, W. Engelhardt, G. Fussman, O. Gehre, J. Gernhardt, G. v. Gierke, G. Haas, M. Huang, F. Karger, M. Keilhacker, O. Klüber, M. Kornherr, K. Lackner, G. Lisitano, G. C. Lister, H. M. Mayer, D. Meisel, E. R. Müller, H. Murmann, H. Niedermeyer, W. Poschenrieder, H. Rapp, H. Röhr, F. Schneider, G. Siller, E. Speth, A. Stäbler, K. H. Steuer, G. Venus, O. Vollmen, and Z. Yü, "Regime of improved confinement and high beta in neutralbeam-heated divertor discharges of the asdex tokamak," Physical Review Letters, vol. 49, pp. 1408-14-12, 1982.

[27] J. W. Connor and H. R. Wilson, "A review of theories of the l-h transition," Plasma Physics and Controlled Fusion, vol. 42, pp. R1-R74, 2000.

[28] J. Lohr, B. W. Stallard, R. Prater, R. T. Snider, K. H. Burrell, R. J. Groebner, D. N. Hill, K. Matsuda, C. P. Moeller, T. W. Petrie, H. S. John, and T. S. Taylor, "Observation of h-mode confinement in the diii-d tokamak with electron cyclotron heating," Physical Review Letters, vol. 60, pp. 2630-2633, 1988.

[29] B. A. Carreras, L. W. Owen, R. Maingi, P. K. Mioduszewski, T. N. Carlstrom, and R. J. Groebner, "Effect of edge neutrals on the low-to-high confinement transition threshold in the diii-d tokamak," Physics of Plasmas, vol. 5, pp. 2623-2636, 1998.

[30] G. S. Xu, B. N. Wan, J. G. Li, X. Z. Gong, J. S. Hu, J. F. Shan, H. Li, D. K. Mansfield, 
D. A. Humphreys, and V. Naulin, "Study on h-mode access at low density with lower hybrid current drive and lithium-wall coatings on the east superconducting tokamak," Nuclear Fusion, vol. 51, p. 072001, 2011.

[31] B. A. Carreras, P. H. Diamond, and G. Vetoulis, "Role of neutrals in the phase transition model," Physics of Plasmas, vol. 3, pp. 4106-4114, 1996.

[32] R. J. Groebner, M. A. Mahdavi, A. W. Leonard, T. H. Osborne, G. D. Porter, R. J. Colchin, and L. W. Owen, "The role of neutrals in high-mode (h-mode) pedestal formation," Physics of Plasmas, vol. 9, pp. 2134-2140, 2002.

[33] R. J. Groebner, M. A. Mahdavi, A. W. Leonard, T. H. Osborne, N. S. Wolf, G. D. Porter, P. C. Stangeby, N. H. Brooks, R. J. Colchin, W. W. Heidbrink, T. C. Luce, G. R. McKee, L. W. Owen, G. Wang, and D. G. Whyte, "Correlation of h-mode barrier width and neutral penetration length," tech. rep., General Atomics, 2002.

[34] R. J. Groebner, M. A. Mahdavi, A. W. Leonard, T. H. Osborne, N. S. Wolf, G. D. Porter, P. C. Stangeby, N. S. Brooks, R. J. Colchin, and L. W. Owen, "Comparison of h-mode barrier width with model of neutral penetration length," Nuclear Fusion, vol. 44, pp. 204-213, 2004.

[35] R. J. Colchin, R. Maingi, M. E. Fenstermacher, T. N. Carlstrom, R. C. Isler, L. W. Owen, and R. J. Groebner, "Measurement of neutral density near the x-point in the diii-d tokamak," Nuclear Fusion, vol. 40, pp. 175-180, 2000.

[36] J. Winter, "Tokamak wall coatings," Plasma Physics and Controlled Fusion, vol. 36, pp. B263-B276, 1994. 
[37] J. Ehrenberg, "Hydrogen and helium recycling in tokamaks with carbon walls," Journal of Nuclear Materials, vol. 162-164, pp. 63-79, 1989.

[38] V. Rohde, A. Kallenbach, R. Neu, and the ASDEX Uprade Team, "Wall retention of deuterium and gaseous impurities in all tungsten asdex upgrade," Plasma Physics and Controlled Fusion, vol. 51, p. 124033, 2009.

[39] R. Neu, G. Arnoux, M. Beurskens, V. Bobkov, and e. a. S. Brezinsek, "First operation with the jet international thermonuclear experimental reactor-like wall," Physics of Plasmas, vol. 20, p. 056111, 2013.

[40] G. M. Wright, D. Brunner, M. J. Baldwin, K. Bystrov, R. Doerner, a. B. L. B. LaBombard, G. de Temmerman, J. L. Terry, and D. G. Whyte, "Comparison of tungsten fuzz growth in alcator c-mod and linear plasma devices," in APS Division of Plasma Physics Meeting, 2013.

[41] A. M. Keesee, Neutral Density Profiles in Argon Helicon Plasmas. PhD thesis, West Virginia University, 2006.

[42] R. A. Stern and J. A. Johnson, "Plasma ion diagnostics using resonant fluorescence," Physical Review Letters, vol. 35, pp. 1548-1551, 1975.

[43] D. N. Hill, S. Fornaca, and M. G. Wickham, "Single frequency scanning laser as a plasma diagnostic," Review of Scientific Instruments, vol. 54, pp. 309-314, 1983. 


\section{Chapter 2}

\section{Helicons}

\subsection{Background}

Helicon plasmas are high efficiency, high density, magnetized, inductively coupled rf sources. Pioneering work in the field was accomplished by Boswell ${ }^{1}$ and $C h e n .{ }^{2}$ Plasma densities of up to $10^{14} \mathrm{~cm}^{-3}$ are achieved with only 100s of Watts of $\mathrm{rf}$ power and magnetic field strengths of 100s of Gauss. The causes for such efficient plasma production are not yet fully understood and are still an active area of research. The ease of plasma production coupled with the ability for continuous operation make helicon sources very versatile and explain their use in a number of different areas of plasma research: space physics, plasma processing, fusion component testing, and plasma propulsion. An excellent review of the history of helicon devices is given by Kline. ${ }^{3}$

Since their invention, the majority of helicon sources rely on noble gas species for fuel stock. However, a few groups have utilized molecular species, specifically hydrogen (deuterium) and nitrogen. ${ }^{4}$ Helicons are also being used as first wall material test stands for fusion devices. ${ }^{5}$ Using fusion relevant fuel sources, such as deuterium, is critical for repro- 
ducing and understanding the plasma-material interactions found in fusion devices.

Much of the previous work in hydrogen helicon sources focused on understanding source efficiency. Molecular dissociation provides a sink for energy absorption, thus achieving high plasma density operation requires careful device tuning. The contribution of both helicon and slow waves has been investigated in linear ${ }^{6-8}$ and toroidal geometries. ${ }^{9,10}$ The effect of the lower hybrid ( $\mathrm{LH})$ resonance on plasma production has also been extensively studied. ${ }^{7,8,11}$ Some basic plasma phenomena have been investigated in hydrogen helicons, including ion beam formation due to current-free double layers ${ }^{12}$ and low frequency electrostatic instabilities. ${ }^{13}$ Diagnostic difficulties, specifically the need for ion selective diagnostics to differentiate between molecular and atomic ions, and the lack of direct hydrogen ion diagnostics, have led to limited use of hydrogen in basic plasma physics experiments.

The stated goal of the Department of Energy Office of Science in its FY 2013 budget is to "Position the U.S. to assert itself in long-pulse, 3D B [sic] science, and fusion materials gaps." 14 Understanding these "fusion materials gaps" requires the construction of sources that simulate tokamak edge conditions. Due to their relatively high densities $\left(\sim 10^{19} \mathrm{~m}^{-3}\right)$ and relatively low temperatures $(\sim 10 \mathrm{eV})$, helicons make excellent test stands for plasma facing materials studies. Additionally, helicons are relatively inexpensive to construct and operate and can operate in long pulse or continuous mode. At least two such stands have been constructed and operated at Oak Ridge National Laboratory. The first, the Mini Radio Frequency Test Facility (Mini-RFTF), investigated hydrogen helicon plasmas in a variety of magnetic configurations. ${ }^{11}$ A second source, the Physics Integration eXperiment (PhIX), is currently under construction, with a planned upgrade to the Prototype High Intensity Source eXperiment (PHISX) to study plasma interactions with tungsten targets, ${ }^{15}$ the proposed first wall material for the International Thermonuclear Experimental Reactor (ITER). 
The high particle fluxes and flows created in fusion test stands are also interesting for plasma propulsion studies. Much of the work originally done on the Mini-RFTF has been applied to the Variable Specific Impulse Magnetoplasma Rocket (VASIMR). ${ }^{16}$ VASIMR relies on a helicon source to create high density plasma, which when coupled with ion cyclotron resonance heating (ICRH), produces a high velocity plasma exhaust. Additionally, because the helicon antenna responsible for plasma creation is located outside the vacuum chamber, plasma erosion of the antenna is eliminated. Elimination of erosion increases the lifetime of the source, overcoming a significant obstacle for space propulsion systems.

\subsection{Physics}

Helicon waves are bounded right-hand circularly polarized electromagnetic waves with $\omega_{c i} \ll$ $\omega \ll \omega_{c e}$, where $\omega_{c i}$ and $\omega_{c e}$ are the ion and electron cyclotron frequencies, respectively. In space such waves are known as whistler waves ( $\mathrm{R}$ waves). The dispersion relation for an $\mathrm{R}$ wave, ignoring collisions, is

$$
\frac{c^{2} k^{2}}{\omega^{2}}=1-\frac{\omega_{p e}^{2} / \omega^{2}}{1-\left(\omega_{c e} / \omega\right) \cos \theta}
$$

where $\omega_{p}$ and $\omega_{c}$ are the plasma $\left(\sqrt{n e^{2} / \epsilon_{0} m_{e}}\right)$ and cyclotron $\left(q B / m_{e}\right)$ frequencies, respectively, and $\theta$ is the angle of propagation with respect to the background magnetic field.

In the $\omega \ll \omega_{c e} \ll \omega_{p e}$ regime, the dispersion relation reduces to

$$
\frac{c^{2} k^{2}}{\omega^{2}}=\frac{\omega_{p}^{2}}{\omega \omega_{c} \cos \theta} .
$$


Letting $k=k_{\text {total }}$ and $k_{\|}=k \cos \theta$, this becomes

$$
k=\frac{\omega}{k_{\|}} \frac{\omega_{p}^{2}}{\omega_{c} c^{2}} .
$$

Evaluating the dispersion relation for the plasma and cyclotron frequencies, and solving for the plasma density gives the simple helicon dispersion relation

$$
n=\frac{k B}{\mu_{0} e}\left(\frac{k_{\|}}{\omega}\right),
$$

where $k_{\|}$is the parallel wavenumber, $k$ is the magnitude of the wavevector, $k=\sqrt{k_{\perp}^{2}+k_{\|}^{2}}$, $B$ is the magnetic field strength, $\mu_{0}$ is the permeability of free space, $e$ is the elementary charge, and $\omega$ is the wave frequency. ${ }^{17}$

Three distinct operating modes exist in helicon sources. As shown in Figure 2.1, transitions between these modes are characterized by discontinuous jumps in plasma density.

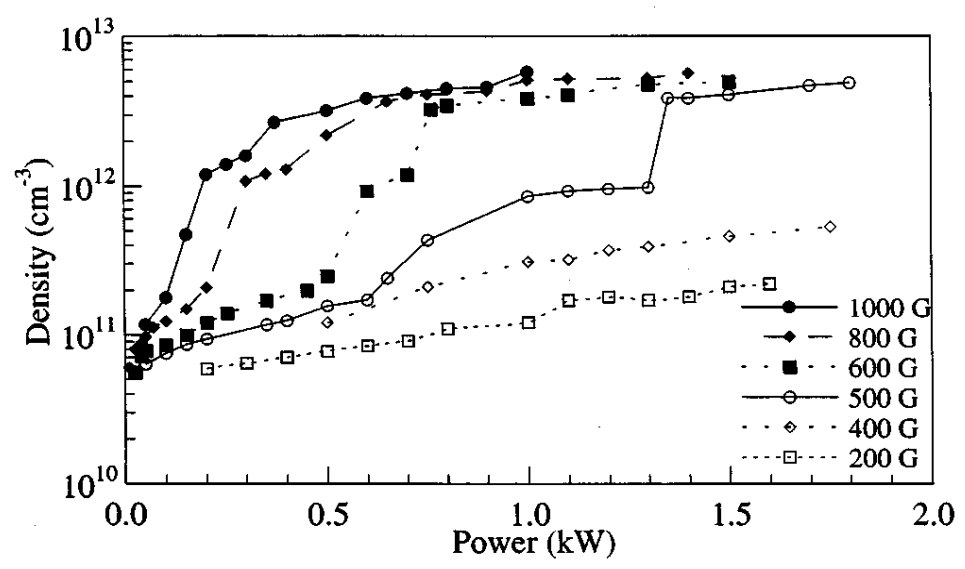

Figure 2.1: Plasma density vs power for a number of different magnetic field strengths. Note the jump in plasma density at a certain power. The threshold for the jump decreases with increasing field. ${ }^{18}$

Transitioning between these modes is achieved by increasing rf power or magnetic field 
strength. The first, the capacitive mode (E mode), is a low density mode in which the plasma is sustained and heated via the capacitive action of the antenna. At low density, $\sim 10^{10} \mathrm{~cm}^{-3}$, the electron skin depth of the plasma, $\delta=c / \omega_{p e}$, where $c$ is the speed of light and $\omega_{p e}$ is the electron plasma frequency, is larger than the source, thus electric fields are not screened out and the plasma is not shielded from the wave. Free charges in the plasma are accelerated due to the capacitive voltage difference between the plasma and the antenna. These accelerated charges ionize background neutrals, sustaining and heating the plasma.

As the density increases, either by increasing the applied rf power or the confining magnetic field, the skin depth of the plasma becomes less than the source size and inductive mode ( $\mathrm{H}$ mode) is achieved. rf electric fields induce currents in the plasma, which sustain and heat the plasma.

Further increases in density result in helicon mode (W mode). In the helicon mode resonant absorption of the rf wave sustains and heats the plasma. The manner in which the wave couples to the plasma is poorly understood and is still an active area of helicon research. ${ }^{19}$ Both collisional ${ }^{20}$ and collisionless ${ }^{9}$ wave damping have been suggested as a possible mechanism for the efficient power deposition in helicon mode. However, Boswell showed that the collisionality needed to be $\sim 1000 \mathrm{x}$ its theoretical value to explain the observed ionization rate. ${ }^{1}$

The power loss per unit volume due to collisions can be estimated as

$$
\frac{P_{\text {coll }}}{V}=n_{e} n_{g} \sum_{j} K_{j} E_{j},
$$

where $V$ is the plasma volume, $n_{g}$ is the neutral density, and $K_{j}$ and $E_{j}$ are the rate coefficients and threshold energies for the various collisional loss processes, ionization, excitation, 
etc. ${ }^{21}$ For Boswell's parameters, $3 \mathrm{eV}$ electron temperature, 1.5 mTorr fill pressure, and plasma densities up to $1 \times 10^{18} \mathrm{~cm}^{-3}$, and plasma volume given by the antenna volume, where plasma is created, ${ }^{1}$ less than $30 \%$ of the power is absorbed via ionizing collisions, see Figure 2.2. Thus, some other mechanism must be responsible for the power absorption and plasma creation.

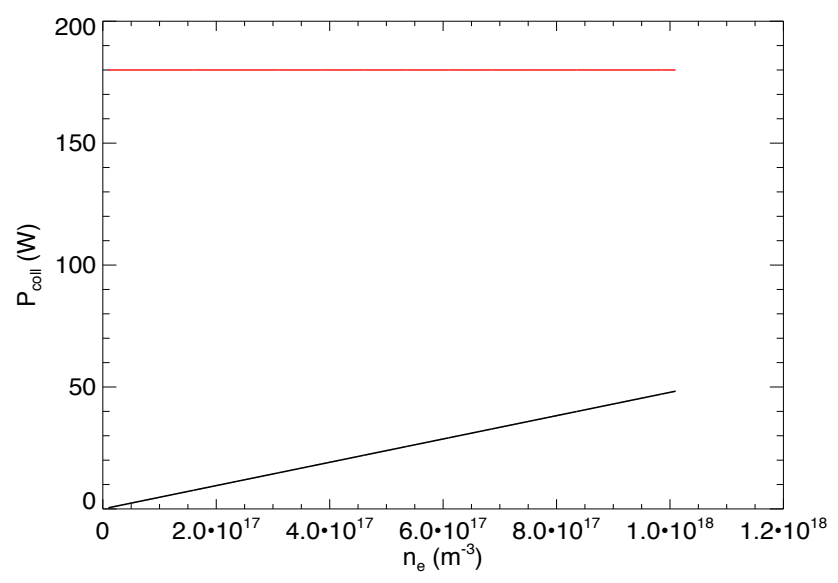

Figure 2.2: Power absorption via collisions for Boswell's parameters. Red curve is input power, $180 \mathrm{~W}$. Black curve is power absorbed by ionizing electron-neutral collisions.

Collisionless wave damping, specifically Landau damping, was been proposed by a number of groups as a possible mechanism to explain the efficient plasma creation. ${ }^{2}$ Landau damping is a process where electrons with speeds near the phase speed of the wave exchange energy with the wave. Electrons moving faster than the wave give up energy to the wave, increasing the wave speed. Electrons moving slower than the wave gain energy from the wave, speeding them up and damping the wave. These electrons are known as resonant electrons, as they satisfy the resonance condition,

$$
v_{e} \sim v_{\phi}=\frac{\omega}{k}
$$


where, $\omega$ is the wave frequency and $k$ is the magnitude of the wave vector. For a Maxwellian distribution of electrons, there will be more electrons with speed less than the phase speed then there are particles with speed greater than the phase speed, see Figure 2.3. The net result of electron interactions with the wave is to damp out the wave and increase the speed of the electrons.

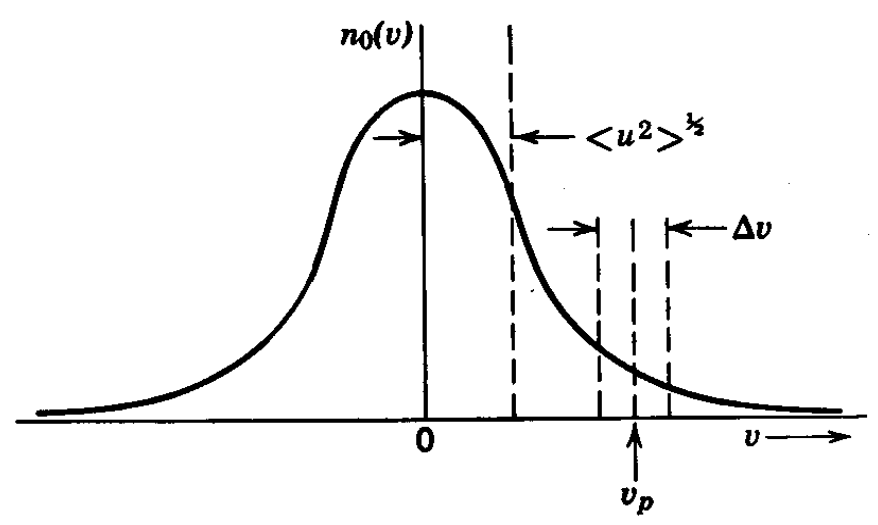

Figure 2.3: Maxwellian distribution of electrons. Only electrons near the phase speed of the wave will interact with the wave resonantly. More electrons a moving slower than the wave than faster than it. Thus, more electrons are taking energy from the wave than are giving energy, thus the wave is damped. ${ }^{22}$

For Landau damping to be an effective means of power absorption, there needs to be a sufficient population of high energy electrons to damp the wave. The ionization rate for argon peaks between 50 and $100 \mathrm{eV} .{ }^{23}$ Chen theorized electrons with this energy would most efficiently couple to the wave. ${ }^{2}$ A number of groups have reported such populations. ${ }^{2,9,24}$ However, distortions in Langmuir probe I-V characteristics caused by rf interference are easily misinterpreted as energetic electrons. ${ }^{25}$ Thus, a sufficient density of energetic electrons needed for collisionless Landau damping to play a significant role has never been conclusively demonstrated. ${ }^{26}$ Another mechanism must be responsible for the efficient power coupling in helicon sources. Such a mechanism is explained in the next section. 


\subsubsection{Lower Hybrid Resonance}

One possible explanation for the efficient power coupling in helicon sources is that it is not the helicon wave that is actually coupling. Rather power is efficiently transferred through damping of slow waves. The cold plasma dispersion relation, including ion motion and collisions, is ${ }^{3}$

$$
\rho^{4}-(\alpha+\beta) \rho^{2}+\alpha \beta+\gamma \delta=0
$$

where $\rho=k_{\perp} c / \omega$, and $\alpha, \beta, \gamma$, and $\delta$ are

$$
\begin{array}{r}
\alpha=\epsilon_{1}-N^{2}-\frac{\epsilon_{2}^{2}}{\epsilon_{1}} \\
\beta=\epsilon\left(1-\frac{N^{2}}{\epsilon_{1}}\right) \\
\gamma=\frac{N \epsilon_{3} \epsilon_{2}}{\epsilon_{1}} \\
\delta=\frac{N \epsilon_{2}}{\epsilon_{1}}
\end{array}
$$

with the index of refraction $N=k_{\|} c / \omega$, and $\epsilon_{1}, \epsilon_{2}, \epsilon_{3}$, the elements of the cold plasma dielectric tensor,

$$
\begin{array}{r}
\epsilon_{1}=1+\sum_{j=e, i} \frac{\omega_{p j}^{2}\left(1+i \frac{\nu_{j}}{\omega}\right)}{\omega_{c j}^{2}-\omega^{2}\left(1+i \frac{\nu_{j}}{\omega}\right)^{2}} \\
\epsilon_{2}=\sum_{j=e, i} \frac{\sigma_{j} \omega_{p j}^{2} \frac{\omega_{c j}}{\omega}}{\omega_{c j}^{2}-\omega^{2}\left(1+i \frac{\nu_{j}}{\omega}\right)^{2}} \\
\epsilon_{3}=1-\sum_{j=e, i} \frac{\omega_{p j}^{2}}{\omega^{2}\left(1+i \frac{\nu_{j}}{\omega}\right)}
\end{array}
$$

where $j$ represents species, $\omega_{c i}$ is the cyclotron frequency, $\omega_{p j}$ is the plasma frequency, $\nu_{j}$ is the total collision frequency, and $\sigma_{j}$ is the sign of the charge, +1 for ions, -1 for electrons. 
For typical helicon parameters, there are two solutions to the dispersion relation. The first, the fast wave, is an electromagnetic wave with characteristics that has been shown experimentally to match those observed in helicon sources. ${ }^{27}$ The second, the slow wave, is a surface wave, which is heavily damped by collisions. Near the lower hybrid resonance,

$$
\frac{1}{\omega_{l h}^{2}}=\frac{1}{\omega_{c i}^{2} \omega_{c e}^{2}}+\frac{1}{\omega_{p i}^{2}+\omega_{c i}^{2}}
$$

or when $\epsilon_{1}$ goes to 0 , the perpendicular wave number of the slow wave goes to infinity, for a collisionless plasma see Figure 2.4. Thus, significant damping of slow waves is expected whenever the antenna frequency approaches the lower hybrid resonance.

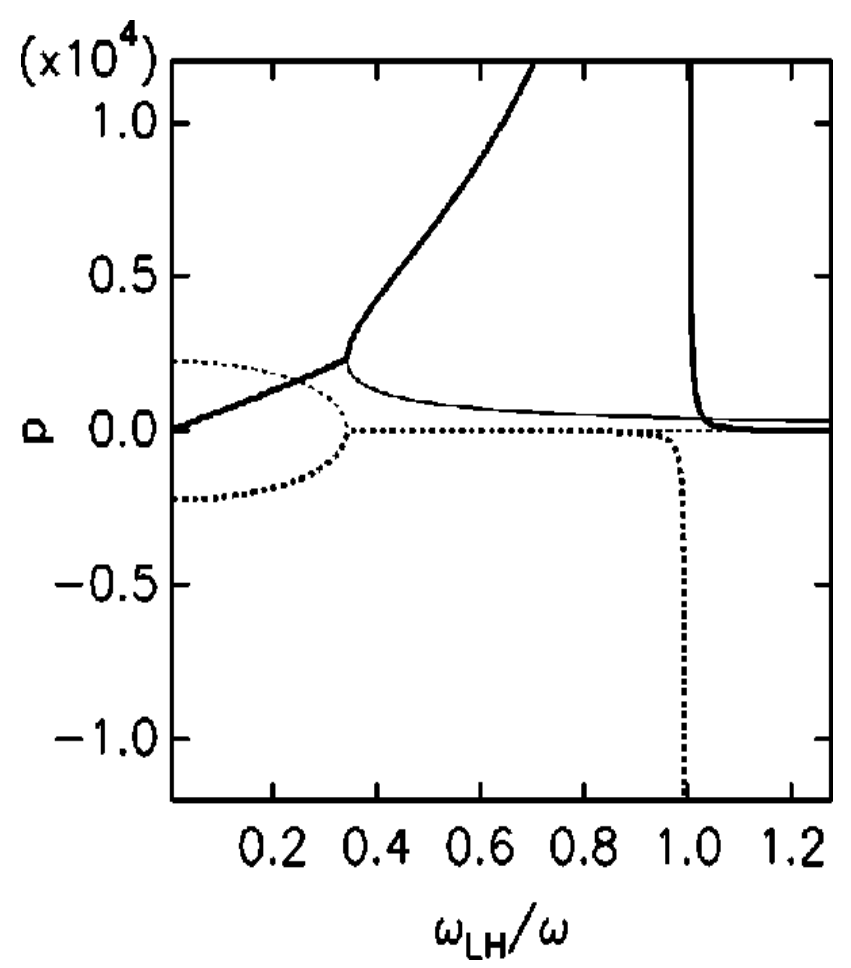

Figure 2.4: Real (solid) and imaginary (dashed) solutions for the cold plasma dispersion relation. At $\omega_{l h} / \omega \sim 0.4$ the real part splits into two solutions, the fast (thin solid) and slow (thick solid) waves. As the wave frequency approaches the lower hybrid frequency the slow wave goes to infinity, indicating a resonance. The fast wave has no resonances, and asymptotically approaches 0 with increasing frequency. ${ }^{28}$ 
Recent measurements at WVU have confirmed the existence of slow waves in the edge region of a high density helicon source, and their role in edge ion heating. ${ }^{29,30}$ The effect of operating near the lower hybrid resonance has been previously investigated in both heavy and light ion helicon plasmas, and has been shown to dramatically affect the ion temperature and plasma production. Kline et al. measured significant perpendicular ion heating when the edge lower hybrid resonance matched the driving frequency in an argon helicon plasma. ${ }^{31}$ Sakawa et al. found that plasma production in hydrogen and deuterium was maximized when the antenna frequency matched the LH resonance, ${ }^{7}$ see Figure 2.5. At no point did the plasma density match that predicted by the simple helicon dispersion relation, Eqn 2.4. This suggests that the slow wave, not the helicon wave, is responsible for the plasma creation.

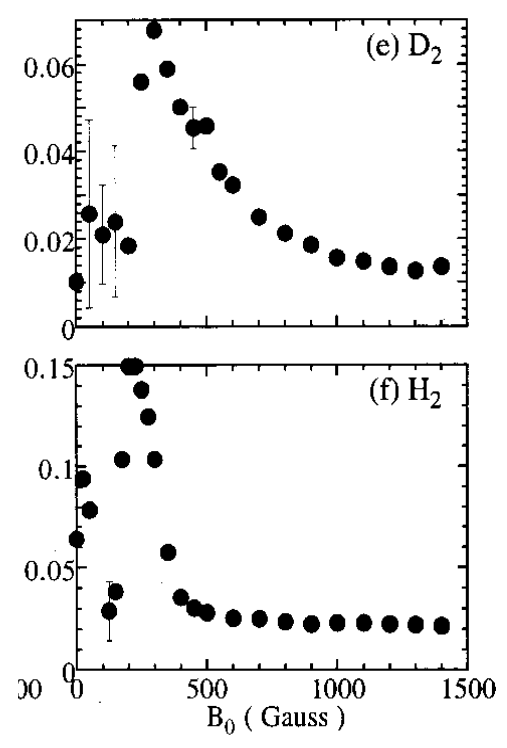

Figure 2.5: Plasma density vs magnetic field in hydrogen and deuterium. Plasma density peaks where the lower hybrid frequency matches the antenna frequency, $\sim 200 \mathrm{G}$ in $\mathrm{H}, \sim 280 \mathrm{G}$ in D. ${ }^{7}$ 


\section{References}

[1] R. W. Boswell, "Very efficient plasma generation by whistler waves near the lower hybrid frequency," Plasma Physics and Controlled Fusion, vol. 26, pp. 1147-1162, 1984.

[2] F. F. Chen, "Plasma ionization by helicon waves," Plasma Physics and Controlled Fusion, vol. 33, pp. 339-364, 1991.

[3] J. L. Kline, Slow Wave Ion Heating and Parametric Instabilities in the HELIX Helicon Source. PhD thesis, West Virginia University, 2002.

[4] C. Biloiu, E. E. Scime, I. A. Biloiu, and X. Sun, "Nitrogen dissociation degree in the diffusion region of a helicon plasma source obtained by atomic lines to molecular band intensities ratio," Journal of Applied Physics, vol. 102, p. 053303, 2007.

[5] R. H. Goulding, J. B. Caughman, T. M. Biewer, J. M. Canik, G. C. Chen, S. J. Diem, L. W. Owen, and Y.-K. M. Peng, "Test stand development at ornl," in Plasma Facing Components 2011 Meeting, 2011.

[6] H. Takeno, Y. Yasaka, O. Sakai, and R. Itatani, "High density central cell plasma production and ion heating by helicon wave damping and its mode conversion into a slow alfvén waves," Nuclear Fusion, vol. 35, pp. 75-85, 1995.

[7] Y. Sakawa, T. Takino, and T. Shoji, "Contribution of slow waves on production of high-density plasmas by $\mathrm{m}=0$ helicon waves," Physics of Plasmas, vol. 6, pp. 47594766, 1999.

[8] H. Kikuchi, Y. Fukui, Y. Sakawa, and T. Shoji, "Damping of waves in helicon-wave 
range of frequency in molecular- and rare-gas plasmas," Physics of Plasmas, vol. 12, pp. 521-526, 2003.

[9] P. K. Loewenhardt, B. D. Blackwell, R. W. Boswell, G. D. Conway, and S. M. Hamberger, "Plasma production in a toroidal heliac by helicon waves," Physical Review Letters, vol. 67, pp. 2792-2794, 1991.

[10] Y. Sakawa, M. Ohshima, Y. Ohta, and T. Shoji, "Production of high-density hydrogen plasmas by helicon waves in a simple torus," Physics of Plasmas, vol. 11, pp. 311-319, 2004.

[11] Y. Mori, H. Nakashima, F. W. Baity, R. H. Goulding, M. D. Carter, and D. O. Sparks, "High density hydrogen helicon plasma in a non-uniform magnetic field," Plasma Sources Science and Technology, vol. 13, pp. 424-435, 2004.

[12] C. Charles, "Hydrogen ion beam generated by a current-free double layer in a helicon plasma," Applied Physics Letters, vol. 84, pp. 332-334, 2004.

[13] M. Light, F. F. Chen, and P. L. Colestock, "Low frequency electrostatic instability in a helicon plasma," Physics of Plasmas, vol. 8, pp. 4675-4689, 2001.

[14] E. J. Synakowski, "Briefing on the administration's proposed funding levels for fes in fy 2013." Online.

[15] R. Goulding, J. Caughman, Y.-K. M. Peng, J. Rapp, T. Biewer, J. Canik, A. Fadnek, D. Hillis, G. Chen, S. Diem, S. Meitner, L. Owen, P. Pesavento, D. Rasmussen, and D. Sparks, "The ornl high-flux helicon source and phix." VLT Conference Call. 
[16] Y. Mori, Study on Light-Ion Helicon Plasma Production with High-Density in Nonuniform Magnetic Field. PhD thesis, Kyushu University, 2004.

[17] F. F. Chen, "Physics of helicon discharges," Physics of Plasmas, vol. 3, pp. 1783-1793, 1996.

[18] P. A. Keiter, E. E. Scime, and M. M. Balkey, "Frequency dependent effects in helicon plasmas," Physics of Plasmas, vol. 4, pp. 2741-2747, 1997.

[19] A. R. Ellingboe and R. W. Boswell, "Capacitive, inductive and helicon-wave modes of operation of a helicon plasma source," Physics of Plasmas, vol. 3, pp. 2797-2804, 1996.

[20] D. G. Miljak and F. F. Chen, "Helicon wave excitation with rotating antenna fields," Plasma Sources Science and Technology, vol. 7, pp. 61-74, 1998.

[21] S. Cho, "A self-consistent model global model of neutral gas depletion in pulsed helicon plasmas," Physics of Plasmas, vol. 6, pp. 359-364, 1999.

[22] P. A. Keiter, Experimental Investigation of Ion Temperature Anisotropy Driven Instabilities in a High Beta Plasma. PhD thesis, West Virginia University, 1999.

[23] M. A. Lieberman and A. J. Lichtenberg, Principles of Plasma Discharges and Materials Processing. Wiley \& Sons, 2005.

[24] R. T. S. Chen and N. Hershkowitz, "Multiple electron beams generated by a helicon plasma discharge," Physical Review Letters, vol. 80, pp. 4677-4680, 1998.

[25] F. F. Chen and D. D. Blackwell, "Upper limit to landau damping in helicon discharges," Physical Review Letters, vol. 82, pp. 2677-2680, 1999. 
[26] I. D. Sudit and F. F. Chen, "Discharge equilibrium of a helicon plasma," Plasma Sources Science and Technology, vol. 5, pp. 43-53, 1996.

[27] M. Light, I. D. Sudit, and F. F. Chen, "Axial propagation of helicon waves," Physics of Plasmas, vol. 2, pp. 4094-4103, 1995.

[28] S. Cho, "The role of the lower hybrid resonance in helicon plasmas," Physics of Plasmas, vol. 7, pp. 417-423, 2000.

[29] R. A. Hardin, Measurement of Short-Wavelength Electrostatic Fluctuations in a Helicon Plasma Source. PhD thesis, West Virginia University, 2008.

[30] E. E. Scime, J. C. Jr., M. Galante, R. M. Magee, and R. A. Hardin, "Ion heating and short-wavelength fluctuations in a helicon plasma source," Physics of Plasmas, vol. 20, p. 032103, 2013.

[31] J. L. Kline, E. E. Scime, R. F. Boivin, A. M. Keesee, and X. Sun, "Slow wave ion heating in the helix helicon source," Plasma Sources Science and Technology, vol. 11, pp. 413-425, 2002. 


\section{Chapter 3}

\section{Experimental Apparatus}

The experiments described in this dissertation were conducted in the Compact Helicon for Waves and Instabilities Experiment (CHEWIE), a small, vertically oriented helicon source. The source was originally designed to study helicon plasmas in a plasma-processing-relevant geometry. However, CHEWIE also been used for diagnostic development and testing. Extensive modifications were made to the design presented in earlier work,${ }^{1-3}$ including a new expansion chamber, smaller glass tube plasma source, and new electromagnets..

As was described in Chapter 2, helicon sources produce high density, $\sim 10^{13} \mathrm{~cm}^{-3}$, low temperature $(10 \mathrm{eV})$ plasmas, conditions very similar to those in the edge of a tokamak. These parameters makes helicons excellent test stands for tokamak first wall component testing and for fusion diagnostic development. Tokamak edge relevant plasma conditions are achieved without the issues associated with performing such experiments in tokamaks, i.e. limited run time and diagnostic access, high heat loads, and intense neutron fluxes, etc.

In this chapter the CHEWIE vacuum chamber, vacuum system, magnetic field structure, and rf systems are described. The principal plasma parameters achieved in the source are provided at the end of the chapter. 


\subsection{Chamber}

The CHEWIE plasma chamber, shown in Figure 3.1, consists of a $61 \mathrm{~cm}$ long, $5 \mathrm{~cm}$ inner diameter Pyrex ${ }^{\mathrm{TM}}$ tube connected to a $20 \mathrm{~cm}$ long, $15 \mathrm{~cm}$ diameter stainless steel chamber. Plasma is created in the tube and then flows into the expansion chamber. Nine $2 \frac{3}{4}$ " Conflat ${ }^{\mathrm{TM}}$ radial ports at two different axial locations, six at one height, three at another, provide diagnostic access. For pressure measurements, an ion gauge is mounted to one of the lower ports and one of the upper ports is used for a Baratron gauge. Four of the upper ports are reserved for laser based diagnostics, with the remaining port used for Langmuir probe access. Two of the four laser ports are equipped with UV grade fused silica windows, allowing for transmission of $205 \mathrm{~nm}$ laser light. The other two windows are boronsilicate (BK7) glass suitable for visible light transmission.

The top of the glass tube terminates in a $6 "$ stainless steel flange. A $2 \frac{3}{4}$ " window in the flange allows axial optical measurements. The top of the tube defines the $z=0$ position and the center of the expansion chamber defines $r=0$. A single fan on the side of the chamber stand cools the antenna section of the glass tube. The bottom of the chamber is connected to the vacuum system.

\subsection{Vacuum system}

A Balzers high-speed turbomolecular drag pump (TMU 520) connected to a Pfeiffer XtraDry 150-2 Dry piston roughing pump provides and maintains high vacuum conditions in the chamber. A pneumatic gate valve separates the chamber from the pumping system and a welded bellows decouples vibrations between the pumps and the chamber. Base pressures are $10^{-7}$ Torr, measured with a Stanford Research Systems ion gauge. Gas is introduced 


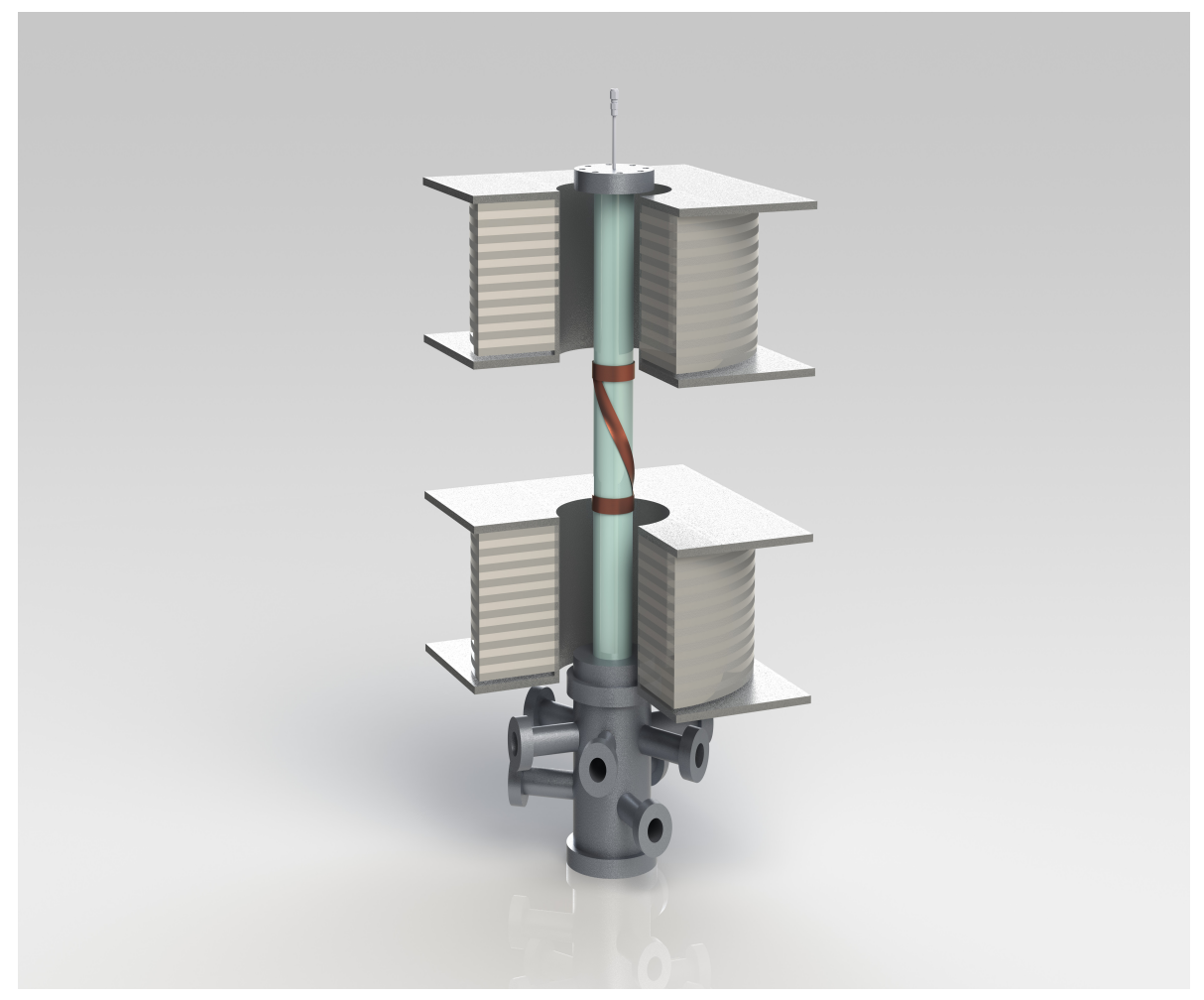

Figure 3.1: The CHEWIE plasma device.

into the system through a feed at the top of the glass tube. A MKS PR4000 power supply and MKS 1179A mass flow controller is used to control the rate of gas feed, typically 0.1-100 SCCM. Discharge operating pressures are 1-50 mTorr, measured with a species independent MKS 179 Type A Baratron gauge.

\subsection{Magnetic Field}

Two new water cooled electromagnets, constructed at WVU, provide steady-state axial magnetic fields of up to $1200 \mathrm{G}$. The magnets are each made of seven sets of $\frac{1}{2}$ " aluminum square tubing wound into seven two-coil "pancakes" of two layers each giving 140 turns per magnet. Each magnet is $21.6 \mathrm{~cm}$ in height, with a $21.6 \mathrm{~cm}$ inner and $75 \mathrm{~cm}$ outer radius respectively. 
The magnets are placed at $-3-18 \mathrm{~cm}$ and $36-57 \mathrm{~cm}$, with respect to the top of the glass tube. An Ametek Sorensen power supply provides current for the magnets. Maximum current is $395 \mathrm{~A}$ at $25 \mathrm{~V}$. A plot of magnetic field strength versus applied current is shown in Figure 3.2. The magnets are cooled continuously during operation using in-house chilled water.

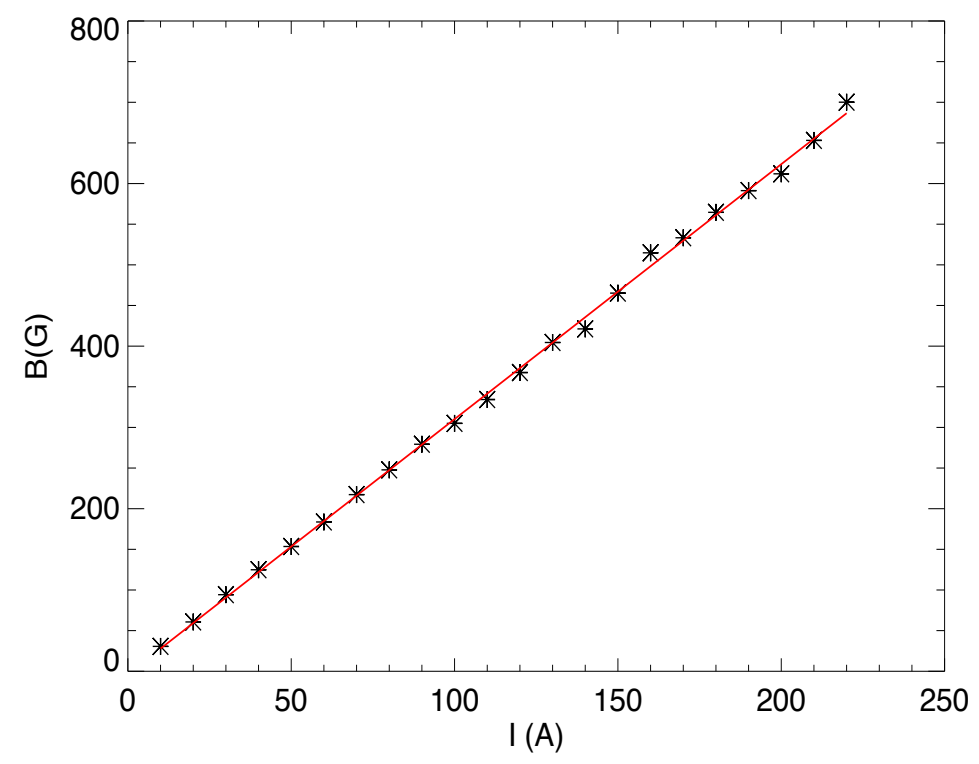

Figure 3.2: Measured magnetic field for applied current in source region, $z \sim 27 \mathrm{~cm}$. Slope of fit line is $3.14 \mathrm{G} / \mathrm{A}$.

In previous CHEWIE experiments, three magnets provided the magnetic field. ${ }^{2}$ However those magnets were made from copper wire with embedded cooling tubes and easily overheated when either a large amount of current was used or the magnets were run continuously. The new magnets with larger tubing and a larger bore allow for more cooling water and longer run times.

The magnetic field strengths cited in this work correspond to the field strength in the 
source region, measured with a Lakeshore 455 DSP gaussmeter. However, the field decreases by $\sim 35 \%$ between the source and TALIF measurement location (see Figure 3.3).

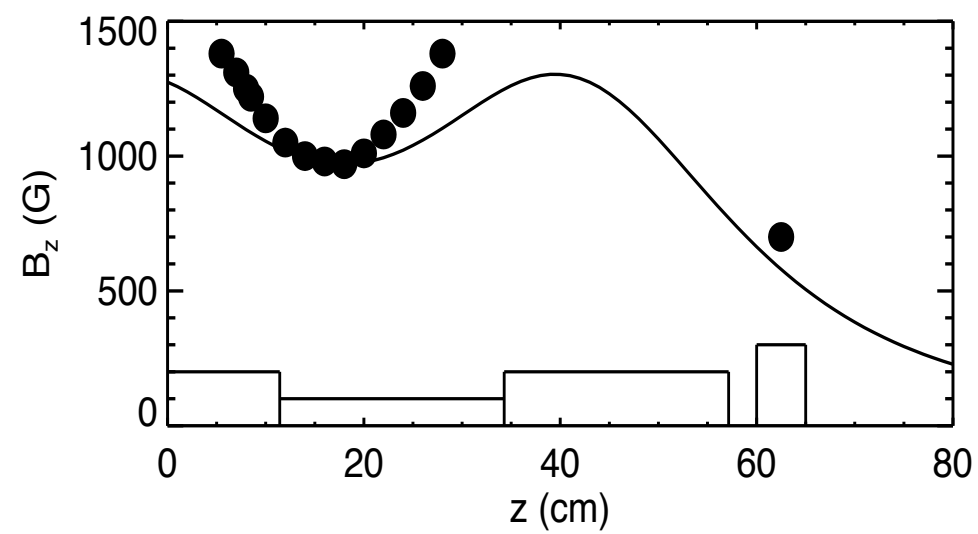

Figure 3.3: Axial magnetic field profile. Points are measurements made with a gaussmeter (see text for model), solid line is an analytical model. Field decreases by $\sim 35 \%$ between source $(\sim 20 \mathrm{~cm})$ and measurement $(\sim 60 \mathrm{~cm})$ regions.

Because the field strength is higher in the source region, it is expected that the plasma density is larger in the source, perhaps by as much as a factor of 10. Due to lack of direct diagnostic access to the source region, it was not possible to verify this assumption. There is a slight radial non-uniformity in the axial magnetic field across the measurement region (Figure 3.4). However, the non-uniformity is less than $\sim 10 \%$, and for the experiments presented here we have assumed the magnetic field is uniform. 


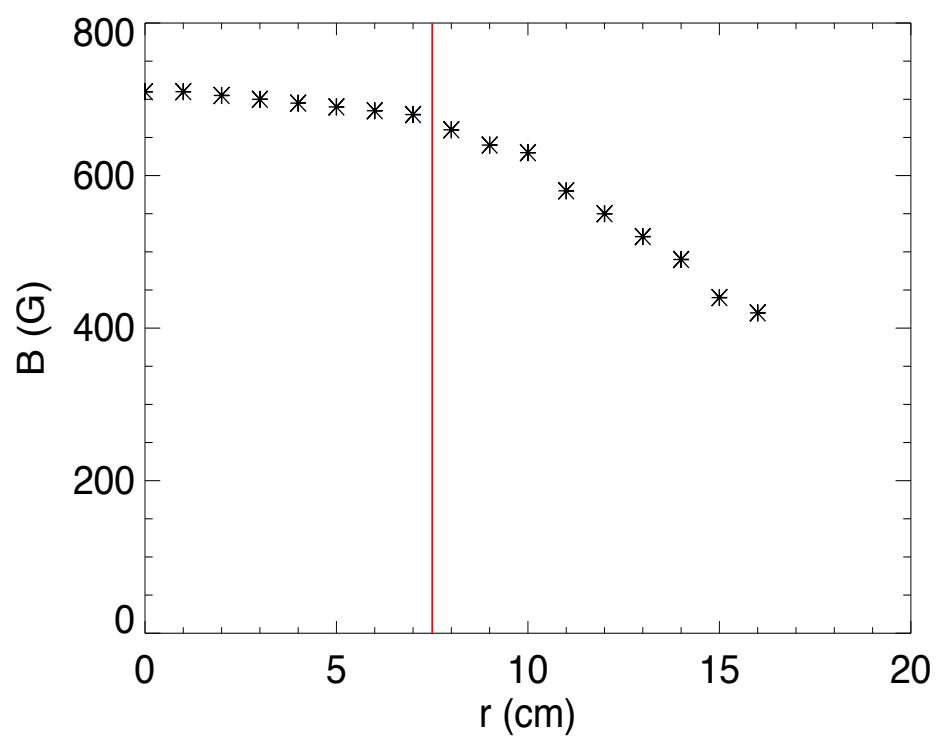

Figure 3.4: Axial magnetic field as a function of radius in the measurement region. Field strength in source $\sim 1200$ G. Red line corresponds to the edge of the TALIF measurement region, $z \sim 60 \mathrm{~cm}$.

\subsection{RF antenna and matching network}

An $18 \mathrm{~cm}, m=1$ right hand helical antenna placed between 10 and $28 \mathrm{~cm}$ creates the plasma in CHEWIE (Figure 3.5). Care is taken to avoid contact between the antenna and the tube as hot spots on the antenna can melt the glass. Two leads from the antenna connect directly to the matching network. $\frac{1}{8}$ " aluminum sheeting attached to the chamber stand provides rf shielding. 


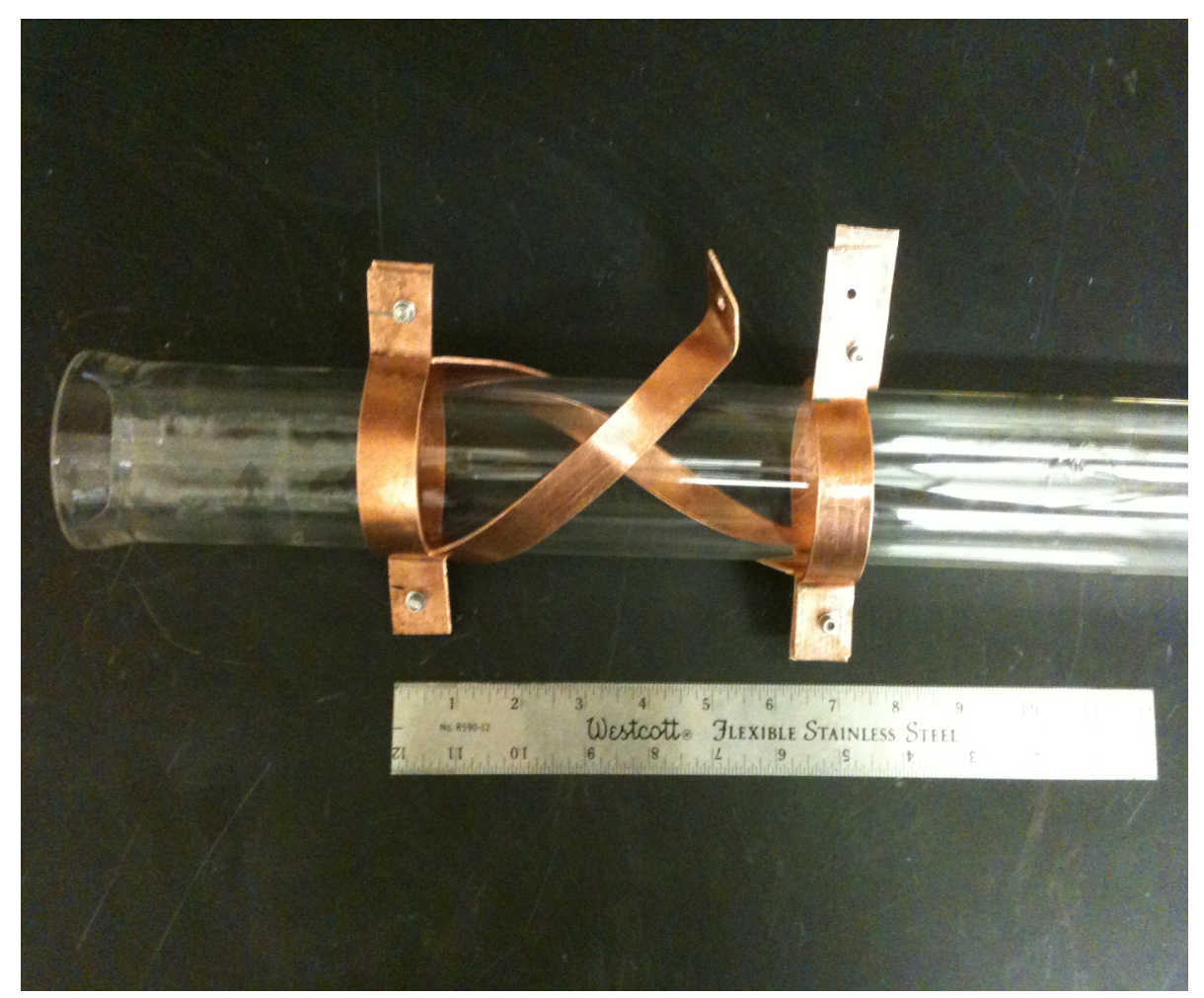

Figure 3.5: $m=1$ helical antenna used to create plasma in CHEWIE. Antenna is made of $\frac{1}{8}$ " thick, 1 " wide copper straps.

Two different rf systems were employed for the experiments. The first consists of a 50 MHz Wavetek model 80 function generator coupled to a ENI 1000 power amplifier. The amplifier is a $36 \mathrm{~dB}$ amp that provides up to $1 \mathrm{~kW}$ of power at frequencies between 6 and 20 MHz. The second system is a Dressler Cesar Model $1350 \mathrm{rf}$ generator, which provides up to $5 \mathrm{~kW}$ of power at $13.56 \mathrm{MHz}$. At high power, $>1 \mathrm{~kW}$, the source is operated in pulsed mode to reduce the heat load delivered to the tube. The rf system is coupled to the matching network with a high-frequency coaxial cable.

A $\pi$-type matching network is used to match the $50 \Omega$ output impedance of the amplifier to the antenna/matching network system. The network consists of one load and one tuning capacitor, see Figure 3.6. Both are Jennings high-voltage vacuum capacitors with tuning 
ranges of $20-2000 \mathrm{pF}$. The tuning capacitor is placed in series with the antenna and the capacitor/antenna series is placed in parallel with the load capacitor. To maximize the antenna coupling to the plasma the real part of the antenna impedance must be matched to the impedance of the amplifier and the imaginary part of the antenna-matching network circuit must be zero. ${ }^{4}$ An excellent discussion of the matching network design and solutions for capacitor values can be found in Keiter ${ }^{5}$ and Balkey. ${ }^{6}$

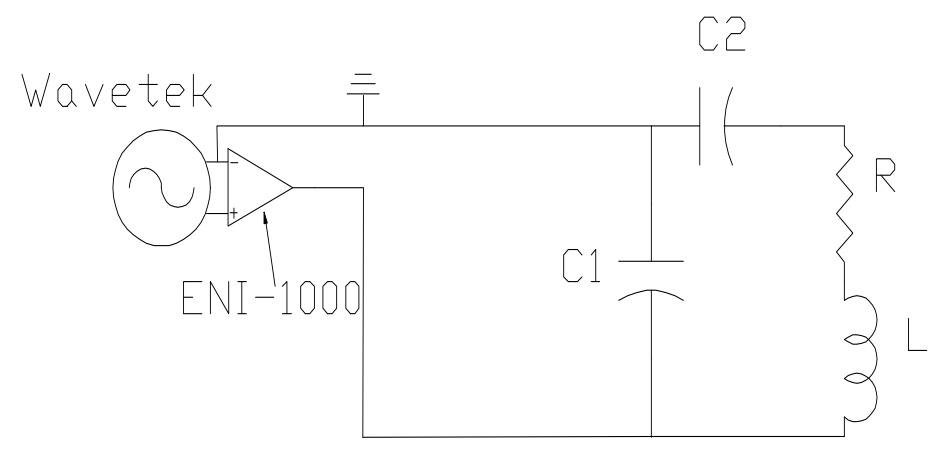

Figure 3.6: Circuit diagram of matching network. $C 1$ and $C 2$ are the load and tuning capacitors, respectively. $R$ and $L$ are the real resistance and impedance of the antenna. The same matching network is used with both power supplies. ${ }^{5}$

\subsection{Plasma Parameters}

Typical operating parameters for CHEWIE are listed in the table below. At least seven different gas species are used in the source, $\mathrm{H}_{2}, \mathrm{D}_{2}, \mathrm{Xe}, \mathrm{He}, \mathrm{N}_{2}, \mathrm{Kr}$, Ar. The table below lists the principal plasma parameters for $\mathrm{H}$ and $\mathrm{Kr}$, the two principal species investigated. Note the large difference in plasma density between the two. This behavior, much higher plasma density in atomic species than molecular species, is systematic in helicon sources. The $\mathrm{H}$ plasma parameters are well representative of the behavior of the other molecular species, and the Kr parameters well describe the other atomic species, except helium. 
Table 3.1: Representative operating parameters in CHEWIE

\begin{tabular}{|c|c|c|}
\hline Parameter (units) & $\mathrm{H}$ & $\mathrm{Kr}$ \\
\hline$B$ (Gauss & \multicolumn{2}{|c|}{$3-1200$} \\
\hline$p(\mathrm{mTorr})$ & \multicolumn{2}{|c|}{$0.5-50$} \\
\hline$T_{e}(\mathrm{eV})$ & \multicolumn{2}{|c|}{$1-10$} \\
\hline$T_{i}(\mathrm{eV})$ & \multicolumn{2}{|c|}{$0.1-1$} \\
\hline$\omega_{c e}\left(10^{9} \mathrm{rad} / \mathrm{s}\right)$ & \multicolumn{2}{|c|}{$0.05-20$} \\
\hline$r_{e}(\mathrm{~cm})$ & $2.0 \times 10^{-3}-2.5$ \\
\hline$r_{i}(\mathrm{~cm})$ & $2.7 \times 10^{-2}-34$ & $2.5 \times 10^{-1}-3 \times 10^{2}$ \\
\hline$n\left(\mathrm{x} 10^{10} \mathrm{~cm}^{-3}\right)$ & $0.1-10$ & $1-100$ \\
\hline$\lambda_{D}(\mathrm{~cm})$ & $7.4 \times 10^{-4}-3.3 \times 10^{-2}$ & $7.4 \times 10^{-4}-2.3 \times 10^{-2}$ \\
\hline$\omega_{p e}\left(10^{10} \mathrm{rad} / \mathrm{s}\right)$ & $0.2-2.0$ & $0.6-18$ \\
\hline$\omega_{c i}\left(10^{5} \mathrm{rad} / \mathrm{s}\right)$ & $0.3-100$ & $3.4 \times 10^{-3}-1.4$ \\
\hline
\end{tabular}




\section{References}

[1] C. Biloiu, E. E. Scime, I. A. Biloiu, and X. Sun, "Nitrogen dissociation degree in the diffusion region of a helicon plasma source obtained by atomic lines to molecular band intensities ratio," Journal of Applied Physics, vol. 102, p. 053303, 2007.

[2] S. C. Thakur, Understanding Plasmas through Ion Velocity Distribution Function measurements. PhD thesis, West Virginia University, 2010.

[3] D. J. Lewis, "Measuring ion velocity distribution functions in a compact, expanding helicon plasma," Master's thesis, West Virginia University, 2008.

[4] F. F. Chen, "Capacitor tuning circuits for inductive loads," tech. rep., UCLA Electrical Engineering Dept. and Institute of Plasma And Fusion Research, 1992.

[5] P. A. Keiter, Experimental Investigation of Ion Temperature Anisotropy Driven Instabilities in a High Beta Plasma. PhD thesis, West Virginia University, 1999.

[6] M. M. Balkey, Optimization of a Helicon Plasma Source For Maximum Density With Minimal Ion Heating. PhD thesis, West Virginia University, 2000. 


\section{Chapter 4}

\section{Diagnostics}

The various diagnostics used in these experiments are presented in this chapter. As half of this dissertation is devoted to the development of a new diagnostic system, only existing diagnostics are presented here. The new diagnostic is described in a separate chapter. Two different classes of diagnostics were used in these experiments: spectroscopy, both emission and absorption, and electrostatic probes.

\subsection{Optical Emission Spectroscopy}

Optical emission spectroscopy (OES) is a widely used plasma diagnostic. Electron-ion and electron-neutral collisions in a plasma result in a distribution of excited states. Radiative decay from these excited states is spectrally dispersed, providing a wealth of information about the plasma, including density, temperature, and composition.

A 1.33 m Czeryn-Turner scanning monochromator (McPherson ${ }^{\mathrm{TM}}$ Model 209) was used as a survey spectrometer to study the emission of the plasma. A diagram of the monochromator optical system is shown in Figure 4.1. 


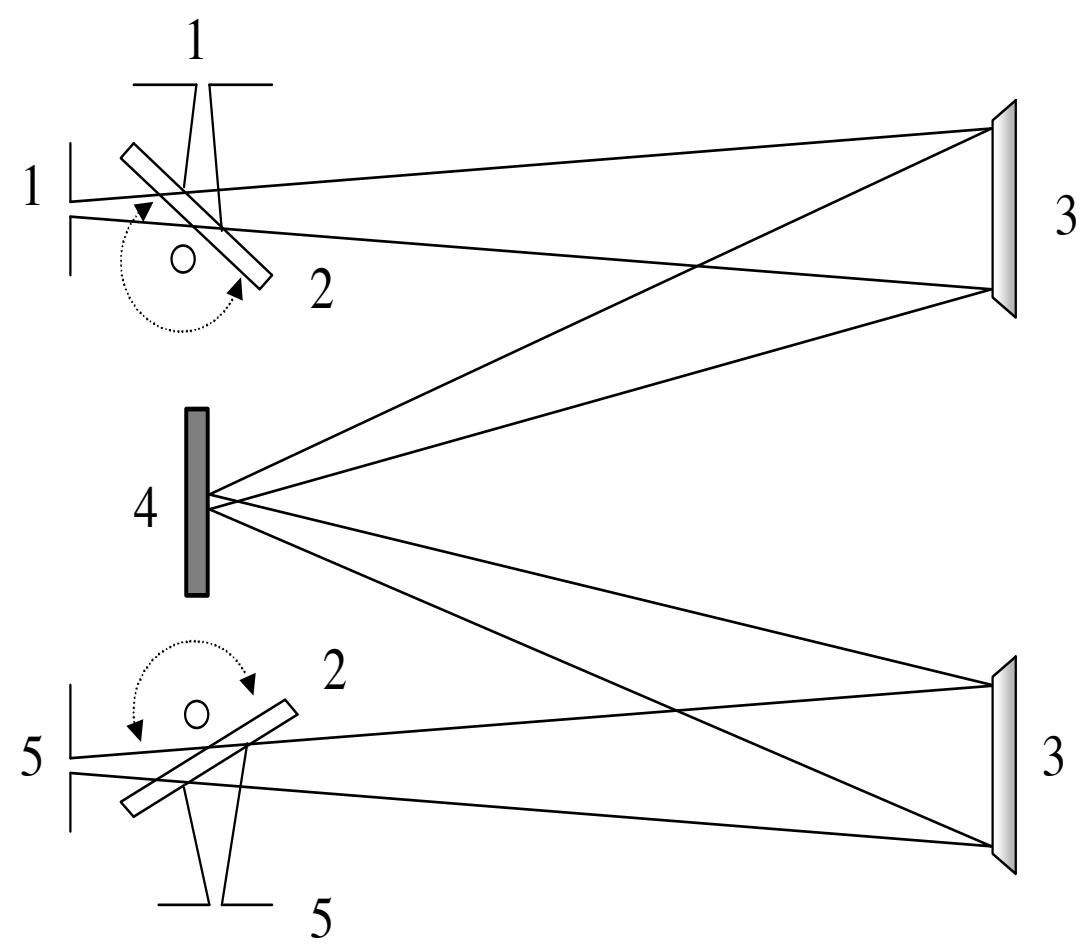

Figure 4.1: Diagram of Czerny-Turner spectrometer. See text for description. ${ }^{1}$

Light enters through the entrance slit (1) and bounces off a folding mirror (2) and onto a concave mirror (3). From the concave mirror it is directed onto the grating (4) where it is dispersed. The dispersed light then bounces off a second concave mirror, off another folding mirror and out the exit slit (5). Light from the exit slit falls onto a CCD camera, Santa Barbara Optics SBIG ST-7XEAI, and the spectrum is recorded on a PC. Typical integration times were 1-100 s, depending on the line under investigation, and the mode of operation, pulsed verses CW. A sample hydrogen spectrum is shown in Figure 4.2. Rotating the grating while keeping the mirror positions and slit width fixed changes the region of wavelength under investigation. Dark signal is recorded then subtracted from light signal, suppressing background noise. The absolute wavelength of the spectrometer is calibrated using a neon pen lamp. Using the known locations of neon emission lines, the grating angle 
is calibrated in terms of wavelength.

The OES collection optics consist of a $5 \mathrm{~cm}$ lens matched to a $200 \mu \mathrm{m}$ multimode optical fiber. The same fiber is used for calibration to eliminate alignment errors arising in matching the fiber to the input of the slit of the spectrometer.

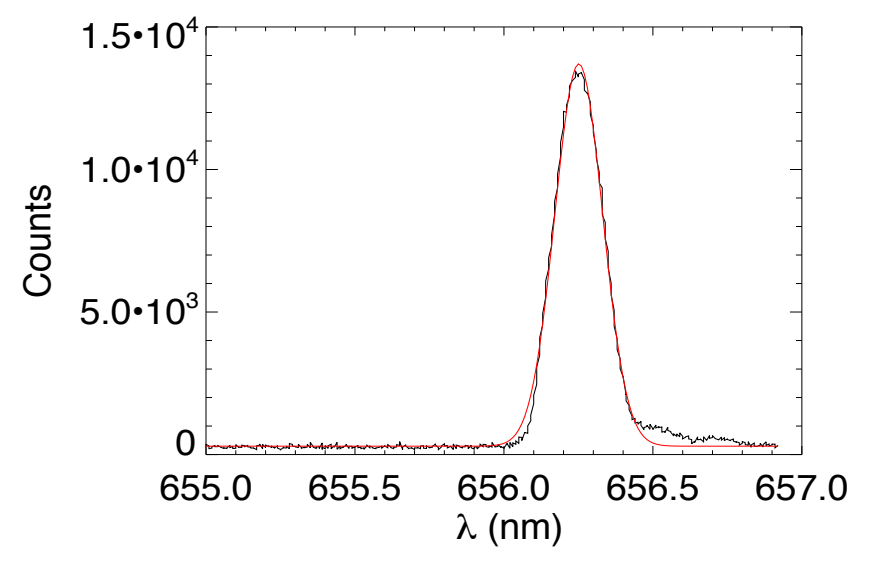

Figure 4.2: Hydrogen emission spectrum.

The dispersive element is a $120 \times 140 \mathrm{~mm}$ grating with 1200 lines $/ \mathrm{mm} .^{2}$ The spectrometer has a spectral resolution of $\sim 100 \mathrm{GHz}$ or $0.1 \mathrm{~nm}$ at $656 \mathrm{~nm}$. This is much larger than the Doppler width of any species under investigation in CHEWIE. For example, the $H_{\alpha}$ line of $0.1 \mathrm{eV}$ neutral hydrogen has a width of $\sim 3 \mathrm{GHz}$. Thus, emission spectra are dominated by instrumental broadening and accurate temperature or flow measurements of any species cannot be made. As such, these spectra are used to determine which, if any emission lines are present in the plasma. 


\subsection{Langmuir Probes}

Langmuir probes are possibly the oldest and simplest of all plasma diagnostics. Invented by Irving Langmuir, ${ }^{3}$ they consist of little more than a conductor inserted into the plasma. When the conductor is biased with respect to the plasma it draws a current. Measurement of the current versus voltage reveals of number of different plasma parameters, most notably the plasma density and temperature.

\subsubsection{Langmuir Probe Theory}

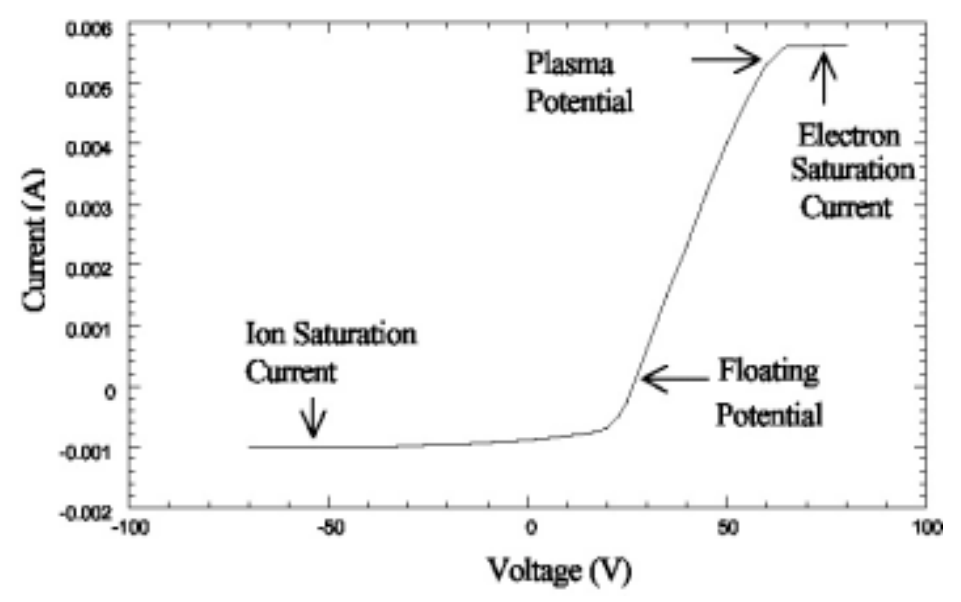

Figure 4.3: Ideal Langmuir probe trace. ${ }^{4}$

A Langmuir probe I-V characteristic, shown in Figure 4.3, can be broken up into three different regions, separated by two characteristic potentials. Region I, the ion saturation region, is when the applied voltage is less than the floating potential, $V_{p}<\Phi_{f}$. The floating potential, $\Phi_{f}$, is the potential at which the probe draws no net current. That is, the electron and ion fluxes to the probe are equal. In region I the probe is biased negative with respect to the plasma and only ions will be collected. Region II, the electron retardation region, 
is when the applied voltage is between the floating and plasma potentials, $\Phi_{f}<V_{p}<\Phi_{p}$. The plasma potential, $\Phi_{p}$, is the potential of the plasma relative to electrical ground. ${ }^{5}$ In region II both ions and electron are collected, however, as the probe is still biased negative with respect to the plasma, only those electrons that have sufficient energy to overcome the electrostatic repulsion of the probe are collected. Because the number of electrons collected by the probe is a function of the electron energy, the electron energy distribution function $(\mathrm{EEDF})$ can be determined from the variation of the current with bias voltage. If the EEDF is Maxwellian, the electron temperature is given by the width of the measured EEDF. Region III, the electron region, is when the applied voltage is above the floating potential, $V_{p}>\Phi_{p}$. In this case, the probe is biased positive with respect to the plasma and only electrons are collected. $^{6}$

Under the assumption of a Maxwellian distribution of electron speeds, the total electric current drawn by the probe is

$$
I=n_{e} e A_{p}\left(\frac{T_{e}}{m_{i}}\right)^{1 / 2}\left[\frac{1}{2}\left(\frac{2 m_{i}}{\pi m_{e}}\right)^{1 / 2} \exp \left(\frac{e V_{0}}{T_{e}}\right)-\frac{A_{s}}{A_{p}} \exp \left(-\frac{1}{2}\right)\right]
$$

where $n_{e}$ is the plasma density, $A_{p}$ is the surface area of the probe, $T_{e}$ is the electron temperature, $V_{0}$ is voltage seen by the plasma (probe voltage minus plasma potential, $V_{p r}-$ $V_{p}$ ), and $A_{s}$ is the surface area of the sheath. The second term is the ion saturation current, which, for an unmagnetized plasma, is ${ }^{5}$

$$
I_{s i}=-e J_{i}=-0.61 e n_{e} A_{p} \sqrt{T_{e} / m_{i}}
$$

When a magnetic field is applied such that the gyroradius of the ions becomes comparable to the characteristic dimension of the probe (length), the ion current drawn by the probe is 
reduced. In this case the ion saturation current is ${ }^{5}$

$$
I_{s i}=-0.49 e n_{e} A_{p} \sqrt{T_{e} / m_{i}} .
$$

Thus, the plasma density can be calculated from the ion saturation current for a known electron temperature.

The electron temperature is determined from the slope of the I-V characteristic. The derivative of the current with respect to the voltage seen by the plasma is

$$
\frac{d I}{d\left(V_{0}-V_{p}\right)}=\frac{e}{T_{e}}\left(I-I_{s i}\right)+\frac{d I_{s i}}{d\left(V_{0}-V_{p}\right)}
$$

Since the ion saturation current is small compared to the current collected above the floating potential, the second term is neglected and the electron temperature is approximated by

$$
T_{e}=e\left(I-I_{s i}\right) / \frac{d I}{d\left(V_{0}-V_{p}\right)}
$$

\subsubsection{Langmuir Probe Design}

Two different Langmuir probes were used to make the electron density and temperature measurements presented here. Aside from the different tip geometries and materials the probes were identical. One had a planar tip (Figure 4.4 top) made fom a $3 \mathrm{~mm}$ radius tungsten disk connected via a $0.05 \mathrm{~mm}$ tungsten wire. One side of the probe is coated with alumina powder so that only one side of the probe draws current. The collecting area of the probe is oriented either upstream or downstream to measure flows in the plasma, i.e. a Mach probe. The other had a cylindrical tip (Figure 4.4 bottom) made from a $0.5 \mathrm{~mm}$ graphite 

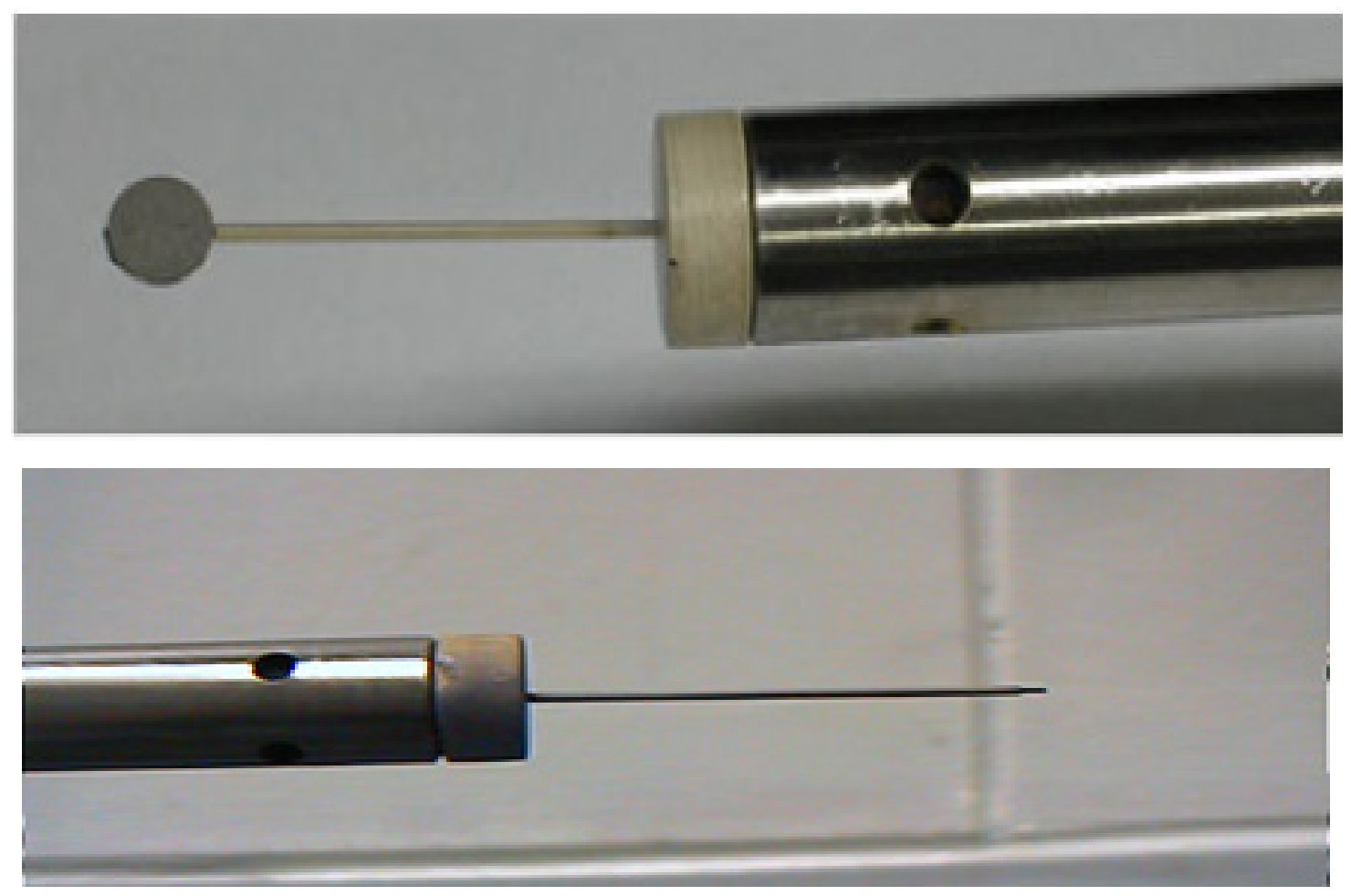

Figure 4.4: Planar (top) and cylindrical (bottom) Langmuir probes. ${ }^{2}$

rod that extended directly into the copper base. For both, a piece of $0.6 \mathrm{~mm}$ ID alumina shaft shielded the probe shaft from the plasma and was secured to a copper plug with a set screw. As shown in Figure 4.5, a $10 \mathrm{nF}$ capacitor decreases the impedance of the sheath, forcing the probe tip to follow oscillations in the plasma potential due to the $\mathrm{rf}$ wave. ${ }^{7}$ These oscillations cause distortions in the I-V characteristic, resulting in an overestimation of the electron temperature. One leg of the capacitor is connected to the copper plug, the other is buried in a thin boron nitride $(\mathrm{BN})$ cap, where it follows the fluctuations in $\Phi_{p}$. The series of rf chokes attached to the copper plug blocks the antenna driving frequency. The chokes are $\frac{1}{4} \mathrm{~W}$, shielded resonant inductors from Lenox-Fugle International Inc ${ }^{\mathrm{TM}}$. The inductor values were chosen such that the entire frequency range of the source is blocked. ${ }^{8}$

The order of the rf chokes is: $26,53,26,13.2$, and $6.8 \mathrm{MHz}$. The end of the rf chokes is 
soldered to a shielded, coaxial probe wire that is attached to the BNC vacuum feedthrough at the far end of the probe shaft. The rf chokes are covered with Thermaflex tubing to protect them from the heat load during plasma operation. The cylindrical probe tip is small enough that plasma perturbation is minimized, however the planar tip is large enough that it can affect the plasma during high density operation.

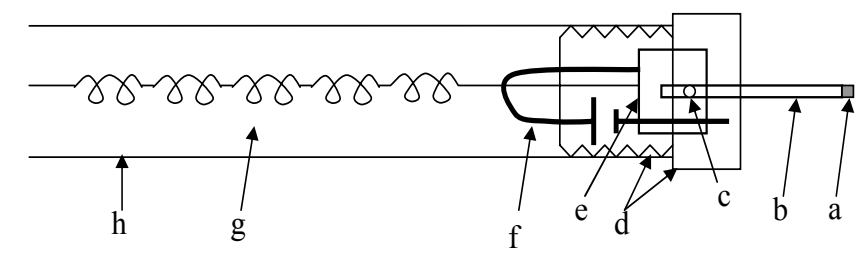

Figure 4.5: RF compensation circuit. a) probe tip, b) alumina shaft, c) set screw, d) threaded boron nitride cap, e) copper base, f) capacitor, g) rf choke chain, h) probe shaft. ${ }^{9}$

The probes are inserted into the plasma a double O-ring sealed, vacuum feedthrough, see Figure 4.6. The double O-ring seal is differentially pumped, so that the probe can be moved radially during operation, allowing the measurement of the radial plasma density profile. The probes are mounted on computer controlled mechanical stages, with resolution of $\sim 0.5$ $\mathrm{mm}$, for automated, high precision scanning.

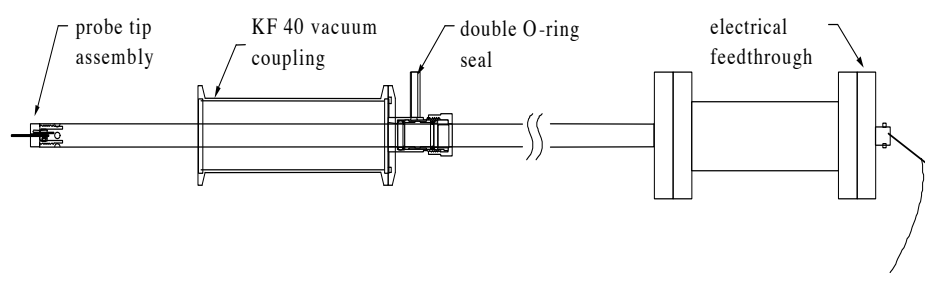

Figure 4.6: Langmuir probe assembly. ${ }^{10}$

Two different data acquisition systems were used to collect Langmuir probe data. When operating in CW mode, a Keithley 2400 high impedance source meter was used to sweep 
the probe bias voltage and measure the drawn current. The bias voltage and current were recorded via a custom built LabWindows ${ }^{\mathrm{TM}}$ interface. In pulsed operation a discrete DC voltage bias is applied to the probe. A time history of the current is recorded as a voltage drop across a $150 \Omega$ resistor, with a digital oscilloscope, see Figure 4.7.

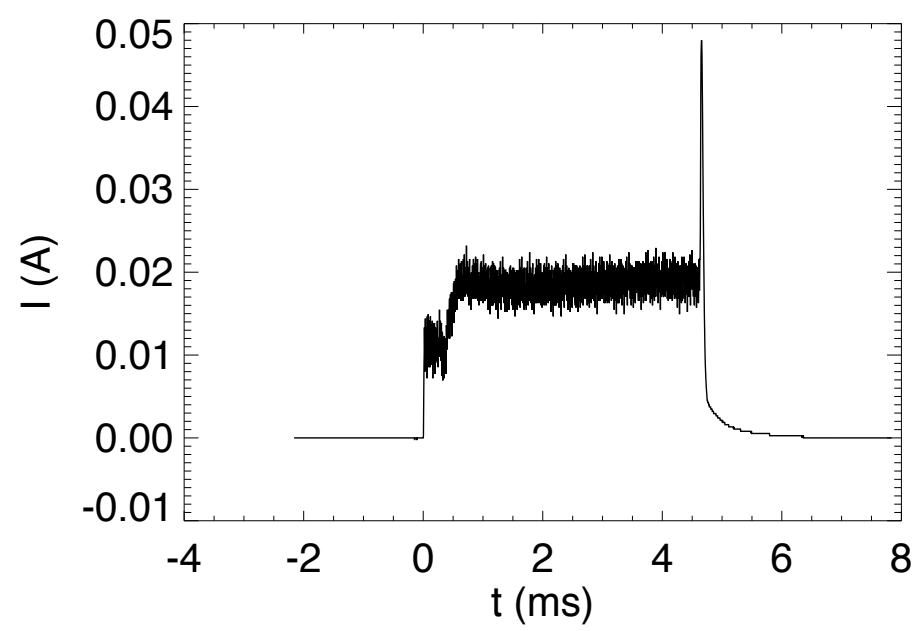

Figure 4.7: Pulsed Langmuir probe time history.

By taking time histories at different bias voltages, I-V characteristics as a function of time are constructed. These characteristics are analyzed individually using the method described above, giving the time evolution of the electron temperature and plasma density.

The planar probe was used for two reasons. The first is that the planar probe can be biased to electron saturation, while the cylindrical probe cannot. As any probe in the plasma is biased to larger and larger positive values, the sheath that forms around the probe tip grows. For planar tip geometries, the increase in sheath area is parallel to the magentic field, thus no additional field lines are intercepted and the collection area does not change appreciably. However, for cylindrical tip geometry the sheath area increases symmetrically about the probe tip. This results in the sheath intersecting more magnetic field lines and increased current is collected. ${ }^{2}$ Because of this, it is impossible to reach electron saturation 
with a cylindrical tip probe in a high density plasma.

The second reason the planar probe was used was it doubles as a Mach probe for measurement of ion flows in the expansion chamber. If there is a preferential flow in the plasma the current drawn when the collector faces upstream will be larger than when the collector faces downstream. The ratio of these currents is related to the Mach number, $M$, in the plasma by,

$$
\frac{I_{u p}}{I_{\text {down }}}=\exp (k M)
$$

where $k$ is an experimentally determined calibration factor. ${ }^{5}$ In the unmagnetized regime, when the ion gyroradius is larger than the probe radius as is the case here, the flow speed is

$$
v=0.746 \sqrt{\frac{Z T_{e}}{m_{i}}} \ln \left(I_{\text {up }} / I_{\text {down }}\right) .{ }^{11}
$$




\section{References}

[1] R. F. Boivin, "Spectroscopy system and basic spectroscopy diagnostics for the helix and leia plasma devices," tech. rep., West Virginia University, 2000.

[2] I. Biloiu, Laser Induced Fluorescence studies of ion acceleration in single and multiple species in expanding helicon plasma. PhD thesis, West Virginia University, 2009.

[3] H. H. Mott-Smith and I. Langmuir, "The theory of collectors in gaseous discharges," Physical Review, vol. 28, pp. 727-763, 1926.

[4] P. A. Keiter, E. E. Scime, and M. M. Balkey, "Frequency dependent effects in helicon plasmas," Physics of Plasmas, vol. 4, pp. 2741-2747, 1997.

[5] I. H. Hutchinson, Principles of Plasma Diagnostics. Cambridge University Press, 2002.

[6] D. N. Ruzic, Electric Probes for Low Temperature Plasmas. American Vacuum Society, 1994.

[7] J. L. Kline, Slow Wave Ion Heating and Parametric Instabilities in the HELIX Helicon Source. PhD thesis, West Virginia University, 2002.

[8] I. D. Sudit and F. F. Chen, "Rf compensated probes for high-density discharges," Plasma Sources Science and Technology, vol. 3, pp. 162-168, 1991.

[9] A. M. Keesee, Neutral Density Profiles in Argon Helicon Plasmas. PhD thesis, West Virginia University, 2006.

[10] P. A. Keiter, Experimental Investigation of Ion Temperature Anisotropy Driven Instabilities in a High Beta Plasma. PhD thesis, West Virginia University, 1999. 
[11] I. H. Hutchinson, "Ion collection by a sphere in a flowing plasma: I. quasineutrality," Plasma Physics and Controlled Fusion, vol. 44, pp. 1953-1977, 2002. 


\section{Chapter 5}

\section{Two-Photon Absorption Laser}

\section{Induced Fluorescence}

In this chapter, two-photon absorption laser induced fluorescence (TALIF) is discussed and the TALIF system designed and constructed at WVU is described. After a brief history, focusing on TALIF in hydrogen, the basic mechanism for TALIF is described. Next, the transition selected for investigation with TALIF is examined, including advantages and disadvantages of the TALIF technique. Broadening mechanisms are discussed, with special attention given to those that are relevant in a magnetic fusion device. Calibration of the TALIF system in krypton is then outlined, including the effect of saturation broadening on calibration. Lastly, the TALIF laser system is described and a theoretical signal-to-noise calculation is presented. 


\subsection{History}

The theory of two-photon absorption was developed in 1931 by Marie Göppert-Mayer, ${ }^{1}$ however it was not until the invention of the laser in the mid-1950's that sufficiently intense light sources became available to test her theory. ${ }^{2}$ This was accomplished by Kaiser and Garrett in 1961 when they measured a two-photon absorption resonance in a doped calcium crystal. ${ }^{3}$ The first TALIF measurements in hydrogen were made by Hänsch et al. in $1975 .{ }^{4}$ They used Doppler-free TALIF to measure the Lamb shift of ground state hydrogen and deuterium. Bokor et al. were the first to make a Doppler broadened measurement in $1981 .^{5}$

In 1990, Kajiwara et al. developed a confocal collection system for use on the Heliotron-E tokamak for edge neutral density measurements. ${ }^{6}$ In 1999, Voslamber proposed construction of a TALIF diagnostic for magnetic fusion devices to measure the ratio of the three hydrogen isotopes (hydrogen, deuterium, and tritium) as a method for real-time fueling measurement. ${ }^{7,8}$ He calculated relevant broadening parameters for a fusion device and defined the laser system necessary to achieve a sufficient signal-to-noise ratio. A system was built and tested on the Pilot Plasma Surface Interface (PSI) experiment at the Institute for Plasma Physics in Berlin based on Voslamber's work. ${ }^{2}$ However, it was never installed on a fusion device. Since the 1990's, TALIF is routinely used to measure neutral hydrogen densities

in low temperature plasma devices. ${ }^{9-13}$ However, no TALIF system has been installed as a regular working diagnostic on a fusion device.

\subsection{Basics}

TALIF is a variation on conventional single-photon LIF. As was described in Chapter One, photon absorption results in the transition of a bound electron from one state to a higher 
energy state. The electron in the higher energy state then spontaneously decays, either back to its original energy state, or to a different one. If the electron decays back to its original state, a photon of energy equal to the one absorbed is emitted. This is known as resonant fluorescence and is a two-level scheme. If the electron decays to a third state, a photon of energy equal to the difference between the second and third states is emitted. This is non-resonant fluorescence and is a three-level scheme.

In TALIF, simultaneous absorption (within $\sim 10 \mathrm{fs}$ ) of two photons pumps the transition, see Figure 5.1. Absorption of the first photon pumps the electron to a "virtual" state. Absorption of the second photon pumps the electron from the virtual state to a real upper state. The virtual state is a linear combination of the wave function of all real atomic levels that combine with the initial state by allowed single-photon transitions. ${ }^{14}$ Therefore, singlephoton linked states are not required for two-photon absorption transitions. The emission for both single- and two-photon LIF is through single photon channels, either back to the original state or to a third state.

Because two photons are absorbed and only one is emitted, TALIF schemes are inherently non-resonant. This is true even for two-level schemes, in which the initial and final states are the same. Therefore, many of the issues associated with resonant fluorescence, specifically scattered laser light dominating the emission signal are avoided, as the absorption and emission are always at different wavelengths. In truth, resonant TALIF is a misnomer, as conservation of momentum prevents direct single-photon decay back to the original state. Collisional coupling between two nearly equal energy states is required for near-resonant TALIF. The need for collisional coupling for the decay pathway presents a number of problems which will be discussed in the next section.

TALIF has a number of advantages over single-photon LIF. Among them are possible 


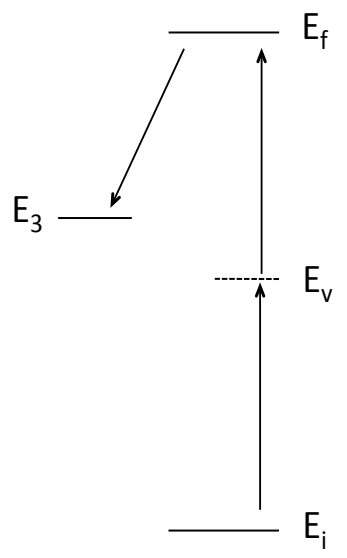

Figure 5.1: Level scheme for two-photon absorption. The dashed line represents a virtual state. Decay is to a third state through a single-photon channel.

direct access to energetic transitions linked to the ground state, pumping otherwise forbidden transitions, and the possibility of performing "Doppler-free" measurements. Additionally, as there are no one-photon transitions at the laser wavelength, optical thickness is not a concern, i.e. the laser travels through the medium unabsorbed until the intensity becomes sufficient to pump the transition, either by focusing or overlapping counter propagating beams.

Most atomic (ionic) ground state transitions lie in the VUV $(<200 \mathrm{~nm})$. It is very difficult to find light sources in the VUV to pump these transitions, and even more difficult to find optical components to handle that light. However, with two-photon absorption, each photon supplies a portion of the energy necessary for the transition. The two photons do not have to have equal frequencies, just so long as the sum of the frequencies equals the transition 
frequency, $\omega_{1}+\omega_{2}=\omega_{T}$. For example, some have proposed using one $193 \mathrm{~nm}$ and one 218 nm photon to pump the $L y_{\beta}$ transition in hydrogen. ${ }^{5}$ Such a scheme requires two lasers. However, two photons of equal frequencies can be produced by a single laser. Using two photons makes it possible to access high energy transitions, such as those from the ground state, that are normally unreachable with single-photon schemes.

For single-photon absorption, a single quantum of momentum is absorbed. Thus the selection rules governing transitions are $\Delta l= \pm 1$, where $l$ is the orbital angular momentum. For two-photon absorption, two quanta of momentum are absorbed and the selection rule becomes $\Delta l=0, \pm 2$. This permits transitions between states that are not coupled by single photon transitions, i.e. $n s \rightarrow m s$ and $n s \rightarrow m d$, where $n$ and $m$ are the principle quantum numbers and $s$ and $d$ are the orbital angular momentum quantum numbers.

These selection rules are satisfied by proper choice of laser polarization. Absorption of two photons with a single polarization satisfies $\Delta L=2$, while absorption of two photons with opposite polarization satisfies $\Delta L=0$. The limits on beam polarization can be exploited when multiple transitions lie near each other. For example, in $\mathrm{H}$, the $1 s{ }^{2} S_{1 / 2} \rightarrow$ $3 d^{2} D_{5 / 2}$ and $1 s^{2} S_{1 / 2} \rightarrow 3 s{ }^{2} S_{1 / 2}$ transitions lie within $0.001 \mathrm{~nm}$ of each other, as seen in Figure 5.2. If absorption were independent of polarization, even a narrow bandwidth laser would pump both transitions simultaneously, resulting in broadening of the absorption line width and an overestimate of temperature and density. However, because of the polarization dependent selection rules, only one transition is pumped for proper polarization selection, thus eliminating the extra broadening. 


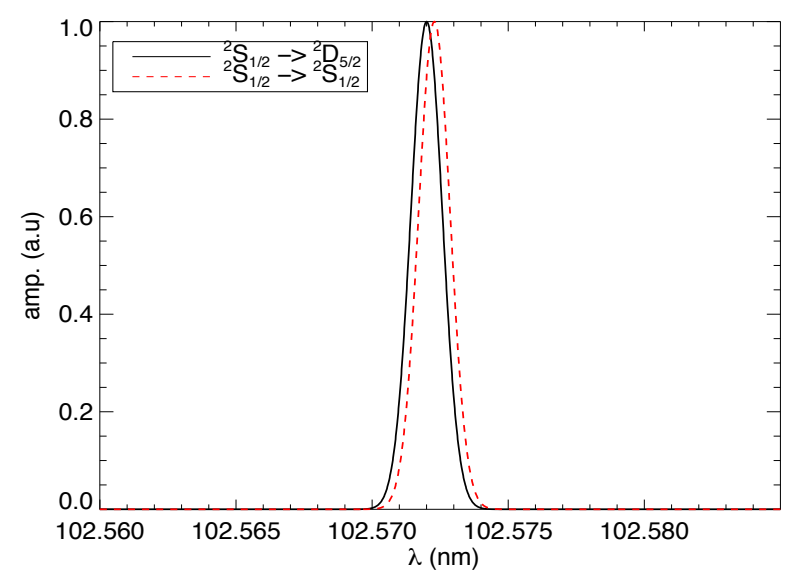

Figure 5.2: Splitting of $1 s^{2} S_{1 / 2} \rightarrow 3 d^{2} D_{5 / 2}$ (solid) and $1 s^{2} S_{1 / 2} \rightarrow 3 s^{2} S_{1 / 2}$ (dashed) transitions for room temperature $(0.03 \mathrm{eV})$ neturals. Absorption wavelengths differ by $\sim 0.001 \mathrm{~nm}$, and would be pumped simultaneously if not for the polarization dependence of TALIF.

\subsubsection{Transition Selection}

There are two ground state transitions in hydrogen that have been previously investigated with TALIF, the $L y_{\alpha}$ transition at $121.6 \mathrm{~nm}(2 \times 243 \mathrm{~nm}$ photons $)$, and the $L y_{\beta}$ transition at $102.5 \mathrm{~nm}(2 \times 205 \mathrm{~nm}$ photons $)$, see Figure 5.3 for both schemes.
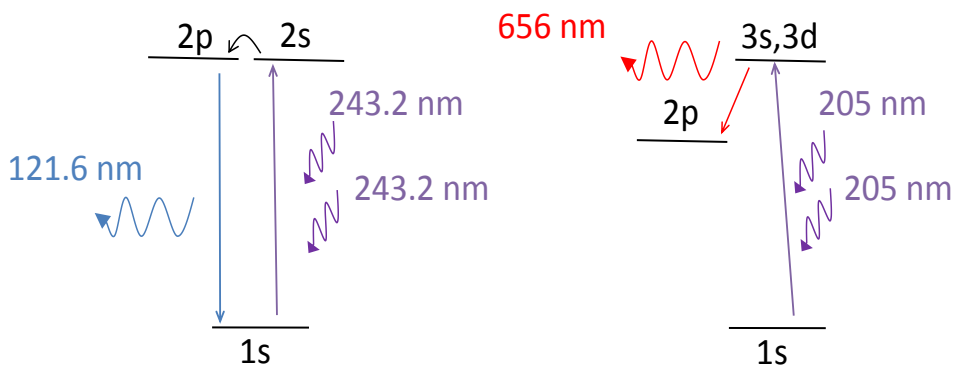

Figure 5.3: Two-photon ground state transitions in neutral hydrogen.

As previously described, a number of groups have utilized the $L y_{\alpha}$ scheme to probe ground state hydrogen in low temperature plasmas, ${ }^{4}$ and significant calculations and modeling have 
been made for its use in tokamaks. ${ }^{8}$ However, the scheme suffers from a number of significant issues. First, the emission is at $121 \mathrm{~nm}$, in the VUV. This makes is very difficult to collect the emitted light, or find conventional optics to handle such an extreme wavelength. Second, and perhaps more troubling is the need for collisional coupling between two states for fluorescence. Absorption of two photons pumps the $1 s{ }^{2} S_{\frac{1}{2}} \rightarrow 2 s{ }^{2} S_{\frac{1}{2}}$ transition. The $2 s$ ${ }^{2} S_{\frac{1}{2}}$ state is metastable, with a lifetime of $\sim 10^{5} \mathrm{~s}$. Collisions couple it to the $2 p^{2} P_{\frac{1}{2}}$ state, which then decays back to the ground state, emitting a $121 \mathrm{~nm}$ photon. Due to the long lifetime of the $2 s$ state, longer detector integration times allow for more collisional coupling to the radiative state, thus increasing the fluorescence yield for constant laser pulse widths. Increasing the integration time also increases the measured background signal. Additionally, as collisions are required to populate the $2 p$ state for decay, the fluorescence yield is a function of both neutral and plasma density. In the region of interest in a tokamak, both can vary by orders of magnitude. Thus, highly accurate knowledge of the plasma density is required to properly determine the neutral density. Collisions can also excite transition not to the $2 p$ state, resulting in smaller fluorescence yields and invalidating any calibration. Lastly, the scheme requires Doppler-free excitation to pump the $\Delta l=0$ nature of the transition. This eliminates direct measurement of the neutral temperature, one of the major benefits of a TALIF measurement.

The $L y_{\beta}$ scheme does not suffer from the need for collisions to couple to a radiative state. Two-photons pump either the $1 s{ }^{2} S_{\frac{1}{2}} \rightarrow 3 s{ }^{2} S_{\frac{1}{2}}$, or the $1 s^{2} S_{\frac{1}{2}} \rightarrow 3 d{ }^{2} S_{\frac{5}{2}}$ transitions, both of which decay directly to the $2 p^{2} P_{\frac{3}{2}}$ state and fluoresce at $656 \mathrm{~nm}$. Thus, the need for collisional coupling is eliminated. The fluorescence is in the visible, making collection very simple as off-the-shelf optical components are readily available. Also, the measurement can be performed either Doppler-free, by pumping the $1 s \rightarrow 3 s$ transition, or Doppler broadened, 
by pumping the $1 s \rightarrow 3 d$ transition.

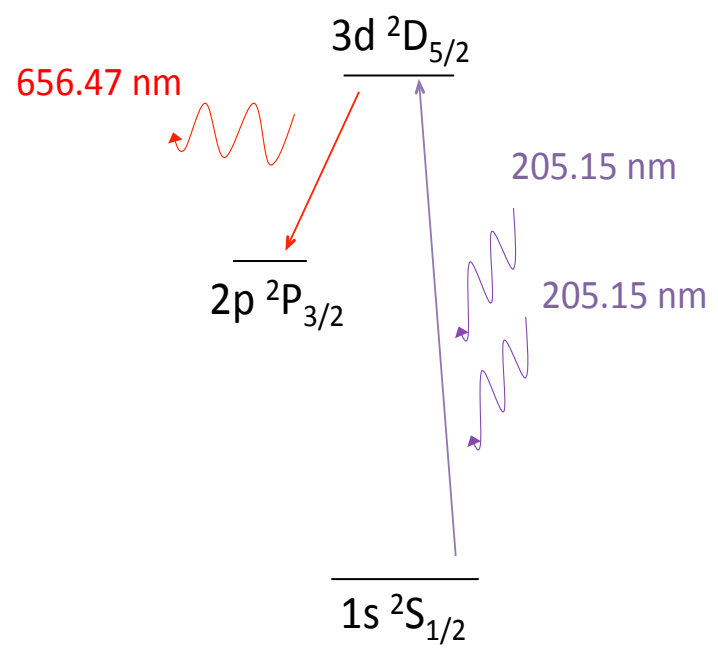

Figure 5.4: Partial Grotrian diagram for the $n=1 \rightarrow n=3$ two-photon transition in hydrogen.

\subsection{Line Broadening}

The lineshape of any collection of absorbing or emitting atoms (ions) has some finite spectral width. Many different mechanisms contribute to this width, including the natural line width, Doppler broadening, Zeeman splitting, Stark splitting, power broadening, and isotopic splitting. For the experiments presented here, Doppler broadening, power broadening, and isotopic splitting are the primary broadening mechanisms. Due to their relevance for tokamak plasmas, Zeeman and Stark splitting are also briefly discussed. The natural line width is negligible compared to the other broadening mechanisms and is therefore omitted 
from this discussion.

\subsubsection{Doppler Effect}

Doppler broadening is the result of random thermal motion of absorbing/emitting atoms. Atoms moving relative to the incident photons will see the frequency of that photon shifted by some amount,

$$
\Delta \nu= \pm \nu_{0} v / c
$$

where $\nu_{0}$ is the unshifted absorption frequency and $v$ is the particle velocity. For a collection of particles in thermodynamic equilibrium, a Maxwellian distribution describes the velocity distribution of absorbing atoms and the absorption spectrum can be fit with a Gaussian function,

$$
f(v)=\sqrt{\frac{m_{A}}{2 \pi k_{B} T}} \exp -\left[m_{A}\left(v-v_{0}\right)^{2} / 2 k_{B} T_{A}\right],
$$

where $m_{A}, T_{A}$ are the atom mass and temperature, and $v_{0}$ is the average atom velocity. The number of emitting atoms at a given frequency is proportional to the intensity of the light emitted at a given frequency, $I(\nu) d \nu \propto f(v) d v$. Thus, the Doppler broadened lineshape is,

$$
I(\nu)=I\left(\nu_{0}\right) \exp \left[-\left(\nu-\nu_{0}\right)^{2} / \sigma_{d}^{2}\right]
$$

where $\sigma_{d}$ is the Doppler width, given by, $\sigma_{d}=\sqrt{k_{b} T / m c^{2}} \nu_{0} \cdot{ }^{15}$ 


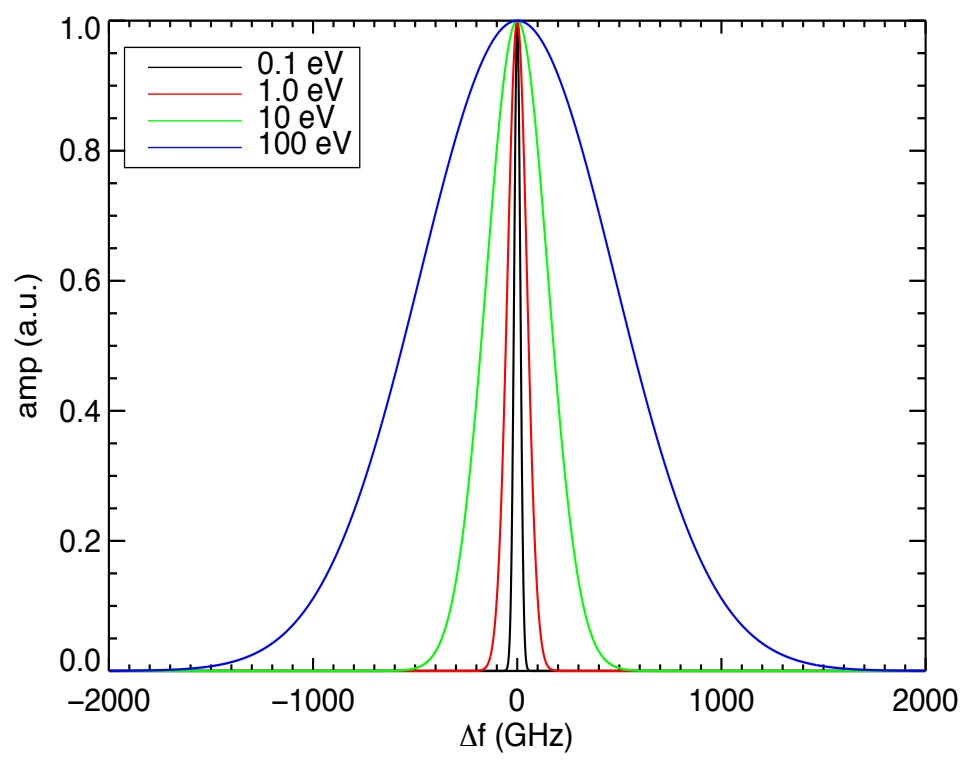

Figure 5.5: Doppler broadened lineshape in hydrogen for four different temperatures. In all cases the Doppler width is larger than the laser line width.

\subsubsection{Zeeman Effect}

Zeeman splitting is the result of the interaction of the magnetic moment of an electron with an applied magnetic field. The Zeeman splitting is given by

$$
\Delta \nu=\frac{1}{h} \mu_{B} B \Delta\left(g m_{j}\right)
$$

where $\mu_{B}$ is the Bohr magneton, $B$ is the magnetic field strength, $g$ is the Lande $g$ factor for each level, and $\Delta m_{j}$ is the change in the $z$ component of the orbital angular momentum. For single-photon absorption, the selection rule on $m_{j}$ is $\Delta m_{j}=0, \pm 1$. For two-photon absorption, two quanta of momentum are absorbed, thus the selection rule becomes, $\Delta m_{j}$ 
$=0, \pm 2$.

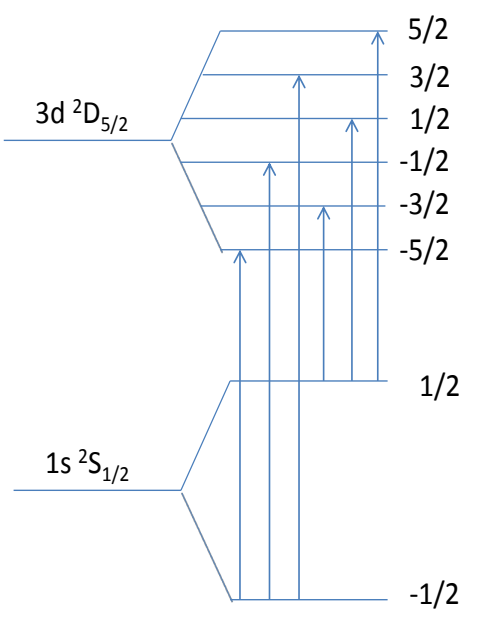

Figure 5.6: Zeeman splitting for $1 \mathrm{~s}{ }^{2} \mathrm{~S}_{1 / 2} \rightarrow 3 \mathrm{~d}{ }^{2} \mathrm{~S}_{5 / 2}$ transition.

For single sided injection, six transitions from $n=1$ to $n=3$ in hydrogen exist, two $\pi\left(\Delta m_{j}\right.$ $=0)$ and four $\sigma\left(\Delta m_{j}=2\right)$. Typically, the splitting between individual Zeeman components is too small to be resolved and the net effect is to broaden of the measured lineshape. As shown in Figure 5.7, for the low fields in CHEWIE the Doppler broadening will be much larger than the Zeeman splitting, even for low neutral temperatures, $<1 \mathrm{eV}$. For a Dopplerfree measurement only two Zeeman split transitions exist, with negligible shift or addition to the measured width. 


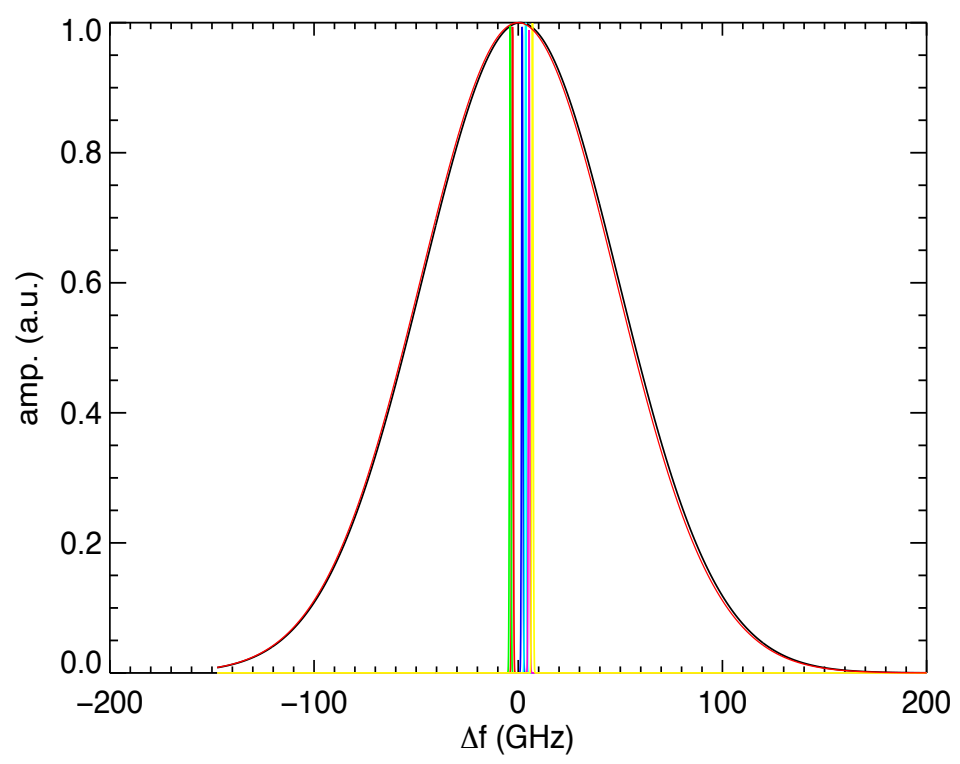

Figure 5.7: Zeeman splitting for the $1 \mathrm{~s}^{2} \mathrm{~S}_{1 / 2} \rightarrow 3 \mathrm{~d}{ }^{2} \mathrm{D}_{5 / 2}$ transition for $1 \mathrm{eV}$ neutrals in a $0.1 \mathrm{~T}$ field.

In a fusion plasma, the larger magnetic field strengths overwhelm the coupling of the orbital and spin angular momenta. In this case, the Zeeman effect becomes the PaschenBack effect, with the splitting given by

$$
\Delta \nu=\frac{1}{h} \mu_{B} B\left(\Delta m_{l}+2 \Delta m_{s}\right)
$$

where $m_{l}$ and $m_{s}$ are the orbital and spin angular momentum quantum numbers. The selection rules for Paschen-Back split transitions are $\Delta m_{s}=0$, and $\Delta m_{l}=0, \pm 2$. For the $1 \mathrm{~s}{ }^{2} \mathrm{~S}_{1 / 2} \rightarrow 3 \mathrm{~d}{ }^{2} \mathrm{D}_{5 / 2}$ transition, nine possible transitions exist, see Figure 5.8, leading to three spectral lines, see Figure 5.9. Even with the larger magnetic field strengths, $>1 \mathrm{~T}$, the expected high neutral temperatures, $>10 \mathrm{eV}$, in a fusion plasma yield a Doppler broadening 
much larger than the Paschen-Back splitting, see Figure 5.9.

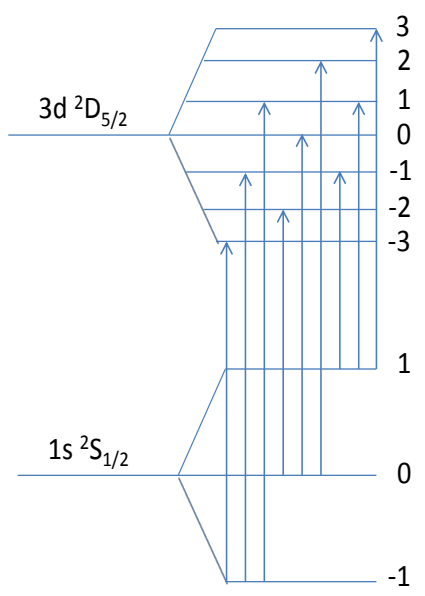

Figure 5.8: Paschen-Back transitions for $1 \mathrm{~s}^{2} \mathrm{~S}_{1 / 2} \rightarrow 3 \mathrm{~d}{ }^{2} \mathrm{D}_{5 / 2}$ transition.

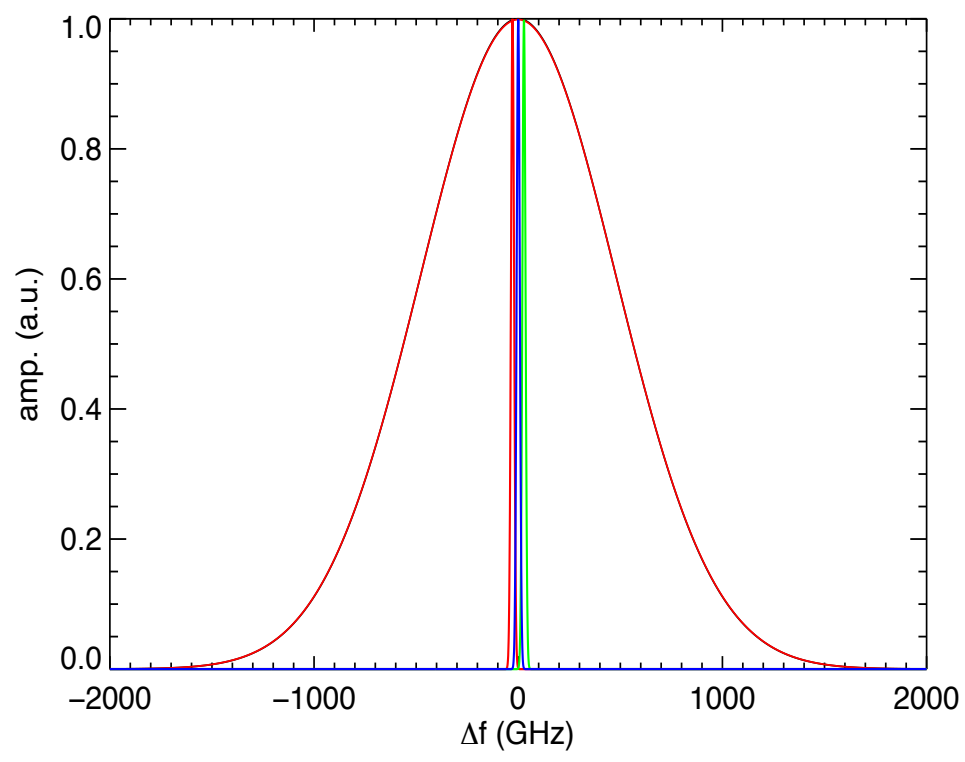

Figure 5.9: Paschen-Back splitting for the $1 \mathrm{~s}^{2} \mathrm{~S}_{1 / 2} \rightarrow 3 \mathrm{~d}{ }^{2} \mathrm{~S}_{5 / 2}$ transition for $100 \mathrm{eV}$ neutrals in a $1 \mathrm{~T}$ field. 


\subsubsection{Pressure/Stark Effect}

Perturbations of emitters by ambient particles reduces the decay time of emitters, resulting in broadening of the natural line width. This is known as collisional or pressure broadening. If the collisions are between like particles, and the emitters are radiatively linked to the ground state, this is known as resonance broadening. Perturbing collisions with like neutral particles is a concern in low temperature, partially ionized plasmas, such as found in CHEWIE. The half-width of a resonance broadened transition is ${ }^{16}$

$$
\Delta \lambda_{1 / 2} \approx 9 \times 10^{-34} \sqrt{\frac{g_{g}}{g_{p}}} f_{g p} \lambda_{p q}^{2} \lambda_{p g} n_{a}
$$

where $g_{g}$ and $g_{p}$ are the statistical weights of the ground and upper states, respectively, $f_{g p}$ and $\lambda_{p g}$ are the oscillator strength and the wavelength of the ground-to-upper state transition, $\lambda_{p q}$ is the wavelength of the transition under investigation and $n_{a}$ is the atomic ground state density. The TALIF scheme under investigation in these experiments directly probes the ground state, thus resonance broadening cannot occur. Additionally, TALIF makes use of a dipole forbidden transition, thus the upper state is not radiatively linked the ground state, again precluding resonance broadening.

Collisions with charged particles introduces an additional effect known as Stark broadening. Plasma ions and electrons produce local electric fields in a plasma, known as microfields. These microfields perturb the states of an emitting atom, resulting in spectral splitting and shifts. In the absence of an applied electric field, electron collisions and ion microfields play the dominate role in causing the splitting. Using a quasi-static approximation values for the splitting of the $H_{\alpha}$ line have been tabulated. ${ }^{17}$ For the densities and temperatures found in CHEWIE the expected splitting is $<10 \mathrm{MHz}$ and is therefore negligible. 
For an externally applied electric field, the problem of Stark splitting reduces to one of elementary quantum mechanics. There is no linear Stark splitting or shift of ground state hydrogen, due to the symmetry of the electron probability distribution. ${ }^{18}$ However, the upper state, $n=3$, splits into nine sub-states. Only four of these sub-states shift, with the largest shift $\sim 10 \mathrm{MHz}$ for an electric field strength of $10 \mathrm{~V} / \mathrm{cm}$, much greater than those expected in the expansion chamber in CHEWIE. Thus, in a low density helicon plasma, Stark splitting is negligible. In tokamaks, where the electric fields are as large as $1000 \mathrm{~V} / \mathrm{cm}$, Stark splitting is a powerful spectroscopic tool. Just as Doppler broadening is utilized to measure absorber/emitter temperature, Stark splitting is used to determine static or induced electric field strengths in the plasma. ${ }^{19}$ Thus, the Stark split spectrum in the edge of a tokamak reveals the magnitude of radial electric field strength or, when used in conjunction with a high energy neutral beam, the magnetic field inside a tokamak.

\subsubsection{Saturation Broadening}

For sufficiently high laser intensities, the absorption rate of a transition becomes comparable to the stimulated emission rate and both are larger than the decay rate. ${ }^{20}$ This means that more electrons are being excited to the upper state than are decaying from it, resulting in a depopulation of the lower state. When this occurs, the power in the wings of the laser line becomes important as there is now sufficient energy in photons far from the laser centroid to excite transitions. Thus, even if the laser peak is tuned far from resonance a finite number of photons in the wings will induce transitions. ${ }^{21}$ The effect of this is to broaden the measured spectrum, resulting in an overestimation of the absorption width. Due to the high laser intensities necessary for TALIF, saturation broadening is a major concern, and is discussed further in the calibration section of this chapter. 


\subsubsection{Isotopic Splitting}

The different masses of isotopes result in shifts of spectral lines in a plasma that is not isotopically pure. If the shift is less than the resolution of the laser, the shift will appear as a broadening of the measured lineshape. In a hydrogen-deuterium-tritium, plasma, the $L y_{\beta}$ lines of hydrogen and deuterium are separated by $\sim 0.07 \mathrm{~nm}$. Tritium and deuterium are even closer, $\sim 0.02 \mathrm{~nm}$. These lines will overlap for neutral temperatures greater than $1.0 \mathrm{eV}$. If all three $\mathrm{H}$ isotopes are present, the resultant absorption lineshape will appear asymmetric (see Figure 5.10). Resolving the different hydrogen isotopes is one of the main goals for TALIF in a fusion device, as it provides a real-time measure of the fusion fueling rate. The krypton TALIF sequence, used for calibration, also displays isotope broadening.

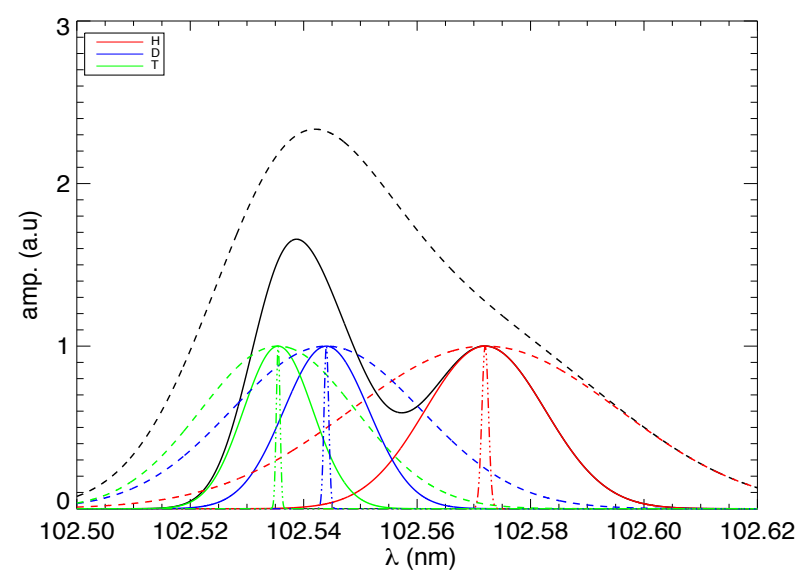

Figure 5.10: Isotopic splitting of the hydrogen $L y_{\beta}$ line for $0.1,10$, and $50 \mathrm{eV}$.

If not properly treated the isotopic splitting of Kr could introduce significant calibration errors. Kr has five stable isotopes, three with significant abundances, $>25 \%$. At low laser energy, the isotope splitting is resolvable and causes the width to deviate from the expected 
width for a room temperature neutral krypton. However, the total lineshape is well fit to a sum of five Gaussians, see Figure 5.11, each with the laser line width, which is comparable to the line width for a cold Kr neutral. Assuming the cross-section for two-photon absorption is the same for each $\mathrm{Kr}$ isotope, fits to the full $\mathrm{Kr}$ TALIF signal provide enough information for an accurate system calibration.

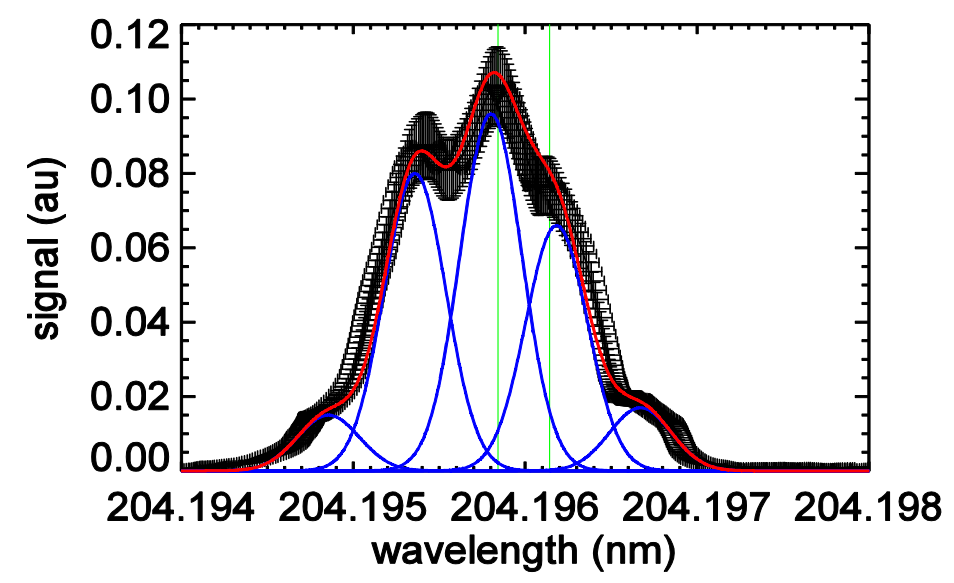

Figure 5.11: $\mathrm{Kr}$ isotope splitting. The measured absorption line is well described by a sum of five Gaussian lineshapes, each with a line width corresponding to the laser line width. The isotope shifts are correct, however, the amplitudes do not always match the relative abundances claimed by the vendor.

\subsection{TALIF Laser System}

The real challenge in doing TALIF is producing UV light to excite a ground state transition. Direct lasing in the UV is very hard. Some technique must be used to produce UV radiation. The WVU TALIF system uses harmonic frequency generation, in multiple stages, to convert long wavelength light to UV. 


\subsubsection{Harmonic Frequency Generation}

Second harmonic generation (SHG) and sum-frequency generation (SFG) are efficient ways to generate high intensity laser light in the UV. Both rely on a change in polarization as input light passes through a non-linear medium. A detailed treatment of harmonic frequency generation theory and techniques is beyond the scope of this dissertation. Thus, only the basics of the technique are presented here. An excellent review can be found in Boyd, which is partially reproduced here. ${ }^{22}$

SHG occurs when a laser with electric field strength

$$
\tilde{E}(t)=E \exp (-i \omega t)+c . c .
$$

where c.c. is the complex conjugate, is incident upon a crystal with non-zero second order susceptibility $\chi^{(2)}$. The electric field creates a nonlinear polarization in the crystal,

$$
\tilde{P}^{(2)}(t)=2 \epsilon_{0} \chi^{(2)} E E^{*}+\left(\epsilon_{0} \chi^{(2)} E^{2} \exp (-i 2 \omega t)+\text { c.c. }\right) .
$$

The polarization produces a term at zero frequency, the first term in the above equation, and a term at the second frequency, the second term. The second term gives rise to radiation at $2 \omega$, shown schematically in Figure 5.12 
(a)

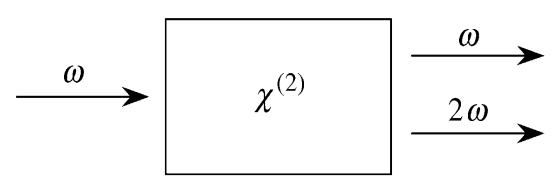

(b)

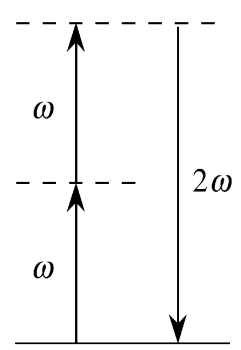

Figure 5.12: Geometry of second harmonic generation (a) and energy level diagram (b). ${ }^{22}$

SFG, shown schematically in Figure 5.13, is analogous to SHG, however two beams of unequal frequencies are used to produce a third beam. An electric field with two distinct frequency components

$$
\tilde{E}(t)=E_{1} \exp \left(-i \omega_{1} t\right)+E_{2} \exp \left(-i \omega_{2} t\right)+c . c .
$$

is incident upon a nonlinear crystal with non-zero $\chi^{(2)}$. The nonlinear polarization is

$$
\begin{aligned}
\tilde{P}^{(2)}(t)=\epsilon_{0} \chi^{(2)}[ & E_{1}^{2} \exp (-2 i \omega t)+E_{2}^{2} \exp (-2 i \omega t)+2 E_{1} E_{2} \exp \left(-i\left(\omega_{1}+\omega_{2}\right) t\right) \\
& \left.+2 E_{1} E_{2}^{*} \exp \left(-i\left(\omega_{1}-\omega_{2}\right) t\right)+\text { c.c. }\right]+2 \epsilon_{0} \chi^{(2)}\left[E_{1} E_{1}^{*}+E_{2} E_{2}^{*}\right] .
\end{aligned}
$$

This interaction produces radiation at the second frequency, $2 \omega$, the sum frequency, $\omega_{1}+\omega_{2}$ and the difference frequency, $\omega_{1}-\omega_{2}$. Through proper phase matching between the incident beams, one process can dominate over the other, and produce intense radiation at a specific frequency. Both SHG and SFG are very efficient processes, so much so that with enough input energy, nearly $100 \%$ conversion efficiency can be achieved. In order to achieve these high input energies typically pulsed seed lasers are required. The WVU TALIF system relies on both techniques. Second harmonic generation converts the fundamental Nd:YAG light 

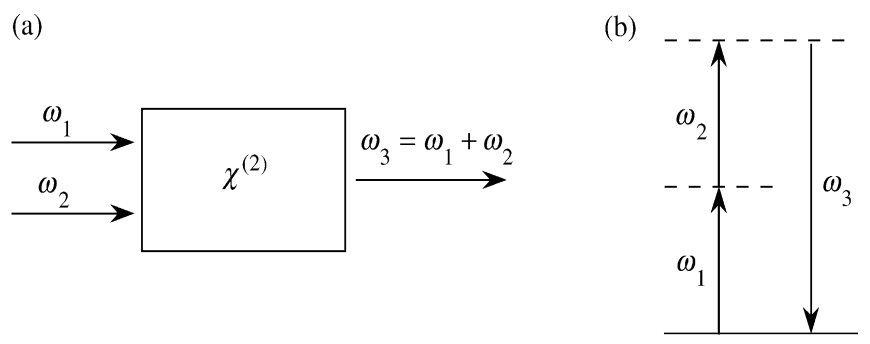

Figure 5.13: Geometry of sum frequency generation (a) and energy level diagram (b). ${ }^{22}$

at $1064 \mathrm{~nm}$ into $532 \mathrm{~nm}$ light to pump the dye laser. Both second harmonic generation and sum-frequency generation produce the UV light from the dye laser light.

\subsubsection{Laser System}

The WVU TALIF system consists of three different lasers. A list of nominal characteristics for each laser is given at the end of the section (see Table 5.1). The first is a Spectra Physics $₫$ Q-switched, frequency doubled, neodymium-doped yttrium aluminum garnet, Nd:YAG, laser. Four $20 \mathrm{~Hz}$ flash lamps, two pre-amp/oscillator lamps and two amplifier lamps produce $1064 \mathrm{~nm}$ photons. A barium borate (BBO) crystal before the output coupler doubles the light pulses to $532 \mathrm{~nm}$ with output energy $~ 0.5 \mathrm{~J}$ per pulse. Beam pointing and divergence are actively controlled via Beam-Lok and D-Lok systems, maintaining energy stability of the $532 \mathrm{~nm}$ beam to $\sim 5 \%$.

The Nd:YAG laser pumps a two-stage Sirah ${ }^{\mathrm{TM}}$ scanning pulsed dye laser running a mixture of Rhodamine B and Rhodamine 101 dyes optimized for $615 \mathrm{~nm}$ output (see Appendix B for dye recipe). The optical layout of the dye laser is shown in Figure 5.14. Two passive dye stages, or cuvettes, produce $615 \mathrm{~nm}$ light. The first is a combination oscillator/preamplifier, with the oscillator output $\sim 10 \mathrm{~mJ}$ and the pre-amp $\sim 30 \mathrm{~mJ}$. The amplifier stage 
increases this power to $150 \mathrm{~mJ}$. The dye laser wavelength is scanned via a Littman-type grating, see Figure 5.14. A tuning mirror scans the light from the first dye stage across the 2400 grooves/mm grating, changing the angle of incidence, and therefore wavelength. The Littman grating also reduces the line width the output beam. ${ }^{23}$

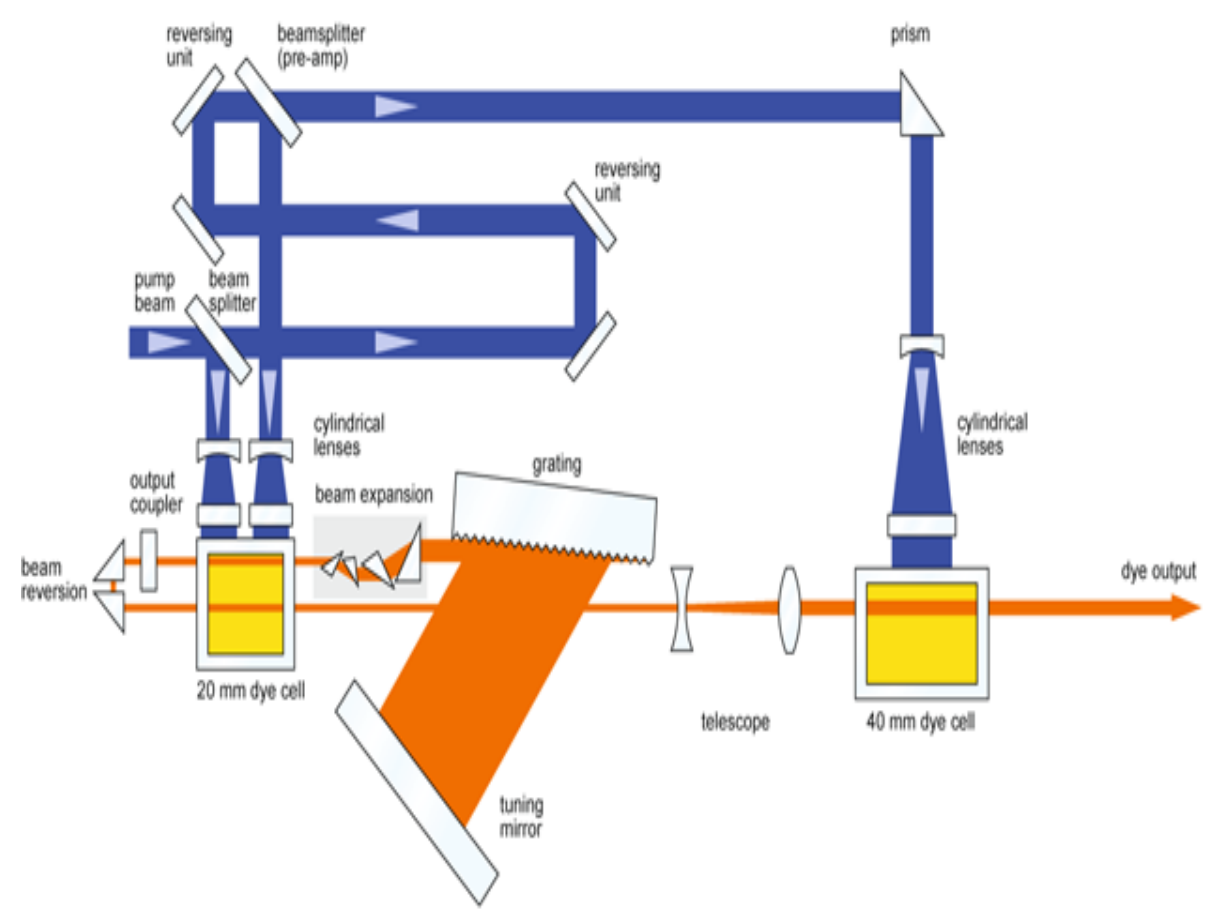

Figure 5.14: Cobra Stretch dye laser optical layout.

The $615 \mathrm{~nm}$ light from the dye laser is frequency tripled to $205 \mathrm{~nm}$ via two non-linear stages. A second harmonic generation (SHG) crystal, SHG-250 BBO Type I, doubles the $615 \mathrm{~nm}$ light to $308 \mathrm{~nm}$ and a sum frequency doubling, SHG-206 BBO Type I, crystal that mixes the primary $615 \mathrm{~nm}$ and $308 \mathrm{~nm}$ light to produce $205 \mathrm{~nm}$ light. Frequency generation requires proper phase matching between the input beams for high intensity output. Angle tuning, adjusting the orientation of the two nonlinear crystals with respect to the propagation direction of the incident beam, is used to maintain the phase match. Active controls scan 
the crystal position during wavelength scans to maintain phase match and maximum energy output.

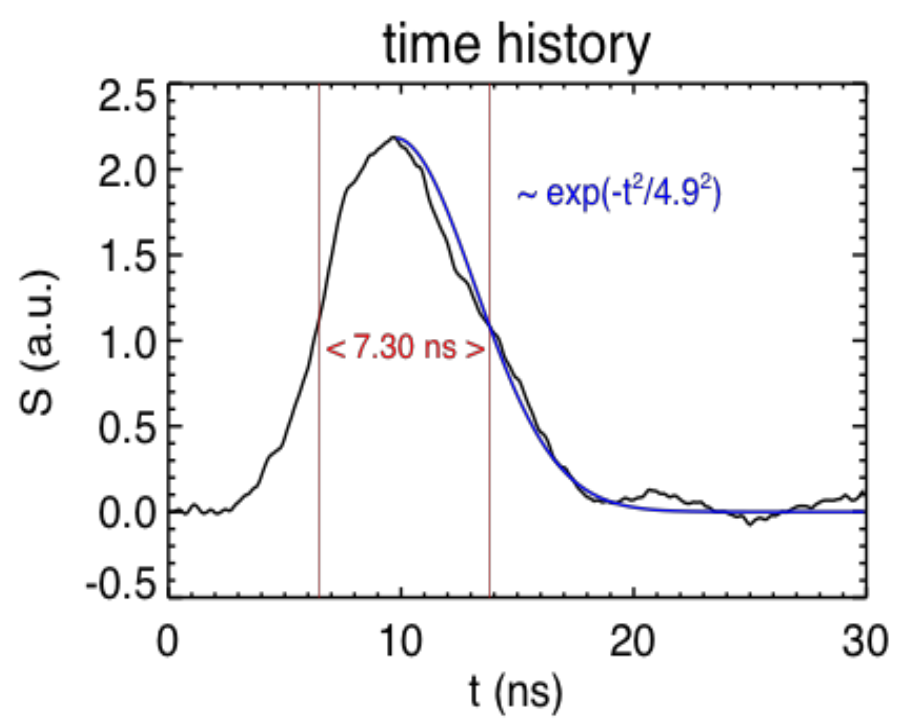

Figure 5.15: Laser temporal shape measured with a fast photodiode.

Two important characteristics of the laser system are the temporal and spatial profiles of the $205 \mathrm{~nm}$ beam. The temporal pulse shape of the $205 \mathrm{~nm}$ beam was measured to be $\sim 7 \mathrm{~ns}$, using a fast photodiode, see Figure 5.15. A knife-edge technique with an iris was used to measure the unfocused beam profile. The beam was partially blocked with the iris and the transmitted energy wave measured with an energy meter. By closing and opening the iris more or less of the beam was blocked, decreasing or increasing the measured energy. Integrating the energy for a number of iris positions gave a measure of the spatial profile of the beam. The integrated beam energy was well described by a Gaussian function with a beam radius of $1.7 \mathrm{~mm}$, see Figure 5.16. Attempts were made to measure the focused beam radius using a knife-edge technique with a razor blade. However, the focused beam was intense enough to cut a channel in the razor blade, distorting the results of the measurement. The width of the laser cut channel provided an estimate of the focused beam width, $\sim 60 \mu \mathrm{m}$. 
The table below outlines the performance of each element in the system.

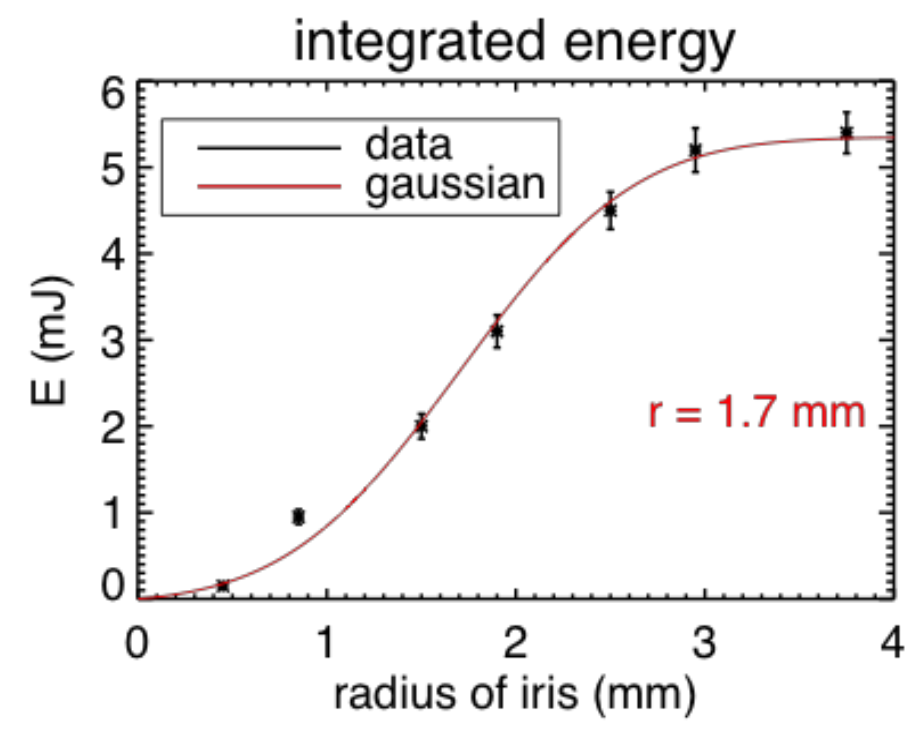

Figure 5.16: Unfocused $205 \mathrm{~nm}$ beam profile measured using a knife-edge technique with an iris. Beam profile is Gaussian with a radius of $1.7 \mathrm{~mm}$.

Table 5.1: Characteristic performance of each element of the TALIF laser system.

\begin{tabular}{|c|c|c|c|}
\hline & Nd:YAG & dye & tripler \\
\hline$E_{\max }(\mathrm{mJ})$ & 500 & 150 & 8.5 \\
\hline Energy variation $(\mathrm{RMS})$ & $<2 \%$ & $5 \%$ & $5-7 \%$ \\
\hline Pulse Width $(\mathrm{ns})$ & $9.7 \pm 0.6$ & $12.1 \pm 0.8$ & $7.1 \pm 0.6$ \\
\hline Line width $\left(\mathrm{cm}^{-1}\right)$ & 1.0 & 0.07 & 0.12 \\
\hline Beam divergence $(\mathrm{mrad})$ & $<0.5$ & 0.5 & $<0.5$ \\
\hline
\end{tabular}

It is interesting to note that the laser has sufficient energy to photo-dissociate and photoexcite water molecules in air. This provides an excellent method for gross alignment without the need for a second species.

The spatial resolution of the system along the beam path is estimated by calculating the Rayleigh length of the focused beam. The minimum radius for a focused Gaussian beam is 
estimated as ${ }^{14}$

$$
w_{0}=\frac{f \lambda}{\pi w_{s}}
$$

where $f$ is the focal length of the lens, $\lambda$ is the laser wavelength, and $w_{s}$ is the radius of the beam on the focusing lens, $\sim 1 \mathrm{~cm}$. For a $25 \mathrm{~cm}$ focal length lens $w_{0} \sim 2 \mu \mathrm{m}$. The Rayleigh length is

$$
z_{R}=\frac{\pi w_{0}^{2}}{\lambda}
$$

For this minimum spot radius the Rayleigh length is $\sim 40 \mu \mathrm{m}$. It is assumed that two-photon absorption only occurs where the beam is sufficiently focused, i.e., within the Rayleigh length. Thus, the spatial resolution of the system is estimated as $<100 \mu \mathrm{m}$.

\subsubsection{Collection Geometry}

Three different collection geometries are possible for the TALIF system. Each presents a number of advantages that make their use in certain situations desirable. The first configuration, shown in Figure 5.17, is known as "perpendicular" collection.

Light is injected into the chamber through one port and emission, localized to the focal spot of the injection lens, is collected through a second port. As shown in Figure 5.17, collection does not need to be exactly perpendicular to the line of injection as fluorescence is emitted isoptropically from the focal spot. Perpendicular collection has the largest collection efficiency as the entire focal spot can be imaged onto the face of the PMT. The measured lineshape is Doppler broadened, therefore both the neutral density and temperature can be directly measured.

The second configuration, shown in Figure 5.18, is known as confocal collection. Unless otherwise noted this was the collection scheme used for the data presented in this dissertation. 


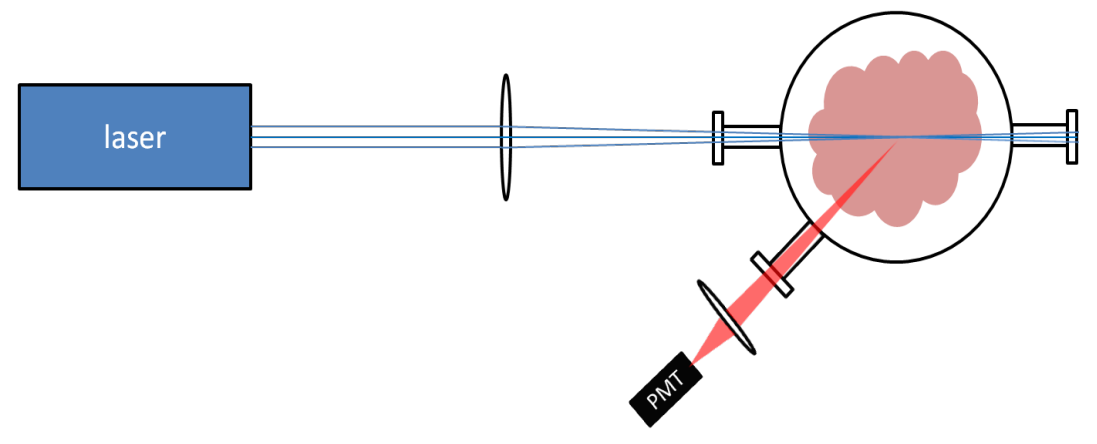

Figure 5.17: Perpendicular collection scheme.

Laser light passes through a small hole in a $45^{\circ}$ turning mirror and is focused through a single port into the chamber. Fluorescence is emitted isotropically from a narrow region centered around the focal spot. As the fluorescence is emitted at the focal length of the focusing lens, the portion of the light that is emitted back along the line of injection will be collimated when it passes through the lens. Rays not emanating from the focal spot will either be focused through the hole in the mirror or not be collimated upon passing through the lens.

The injection and collection optics are one-in-the same, thus the system is self-aligning. Moving the location of the focal spot in the plasma moves the excitation and emission location simultaneously. This technique is used to easily measure profiles in the plasma. Again, the measured lineshape is Doppler broadened, thus density and temperature are measured. 


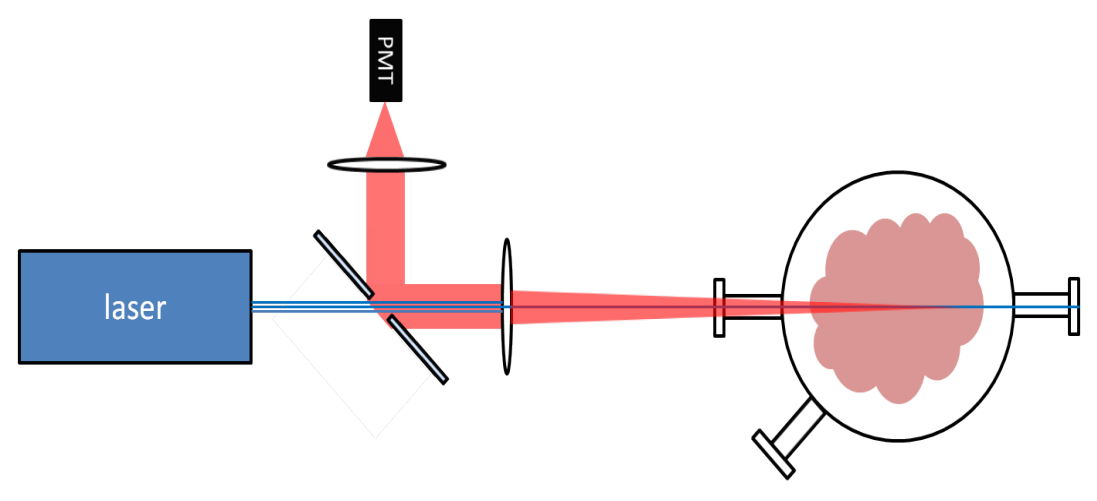

Figure 5.18: Confocal collection scheme.

The final configuration, shown in Figure 5.19, is known as counter-propagating. Light is injected through a single port and is reflected back on to itself by a mirror on the opposite side of the chamber. At the point of overlap of the injected and reflected beams the intensity is sufficiently high to excite measurable TALIF signal, which is collected from a perpendicular view with optics focused onto the point of overlap. The injected and reflected beams are not focused, therefore they do not have high enough intensity to excite measurable signal individually and the emission is limited to the point of overlap. For a small chamber, in which the pulsed beam length is larger than the chamber size, the point of overlap will be the entire chamber diameter. In a large tokamak, the pulsed beam length is smaller than the chamber, and the overlap region must be created by splitting the incident pulse into two pulses one of which is time delayed relative to the other to create an overlap region at the desired measurement location. The counter propagating configuration can also be created by splitting a single beam into two, then injecting each beam from opposite sides of the chamber, eliminating the need for a time delay. In the counter-propagating geometry all 


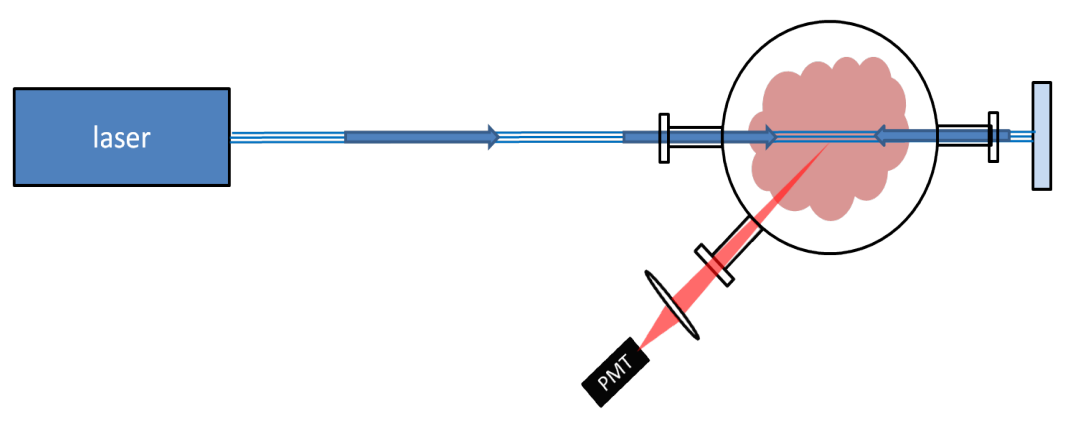

Figure 5.19: Counter-propagating collection scheme.

ground state atoms can be excited, regardless of their velocity, because of the cancellation of the first-order Doppler shift in the absorption of two counter-propagating photons.

To understand Doppler-free excitation, consider an atom moving with velocity $\vec{v}$ in the lab frame. In the reference frame of the moving atom, the frequency $\omega$ of an EM wave with wave vector $\vec{k}$ is Doppler shifted to

$$
\omega^{\prime}=\omega-\vec{k} \cdot \vec{v} .
$$

The resonance condition for the simultaneous absorption of two photons is

$$
\left(E_{f}-E_{i}\right) / \hbar=\left(\omega_{1}^{\prime}+\omega_{2}^{\prime}\right)=\omega_{1}+\omega_{2}-\vec{v} \cdot\left(\overrightarrow{k_{1}}+\overrightarrow{k_{2}}\right),
$$


with $E_{f}$ and $E_{i}$ the energies of the final and initial states, and $\omega_{1}$ and $\omega_{2}$ the frequencies of the input lasers. If the two photons are absorbed out of two lasers with equal frequencies, $\omega_{1}=\omega_{2}=\omega$, which travel in opposite directions, then $\overrightarrow{k_{1}}=-\overrightarrow{k_{2}}$ and the Doppler shift of the two-photon transition becomes zero. This means that all atoms, independent of their velocity, absorb at the same sum frequency, $\omega_{1}+\omega_{2}=2 \omega{ }^{14}$

As the counter-propagating scheme is free of Doppler broadening only the neutral density is measured. Because there is no Doppler broadening, there is no need to scan the laser and the acquisition time is greatly reduced. Using a counter-propagating geometry it may be possible to resolve the neutral density between dynamic events in a tokamak, such as Edge Localized Modes (ELMs). ELMs are discrete releases of energy from the edge of the plasma, and typically occur on the $1 \mathrm{kHz}$ time scale. Understanding how the neutral density in the edge changes between ELMs is one of the open questions in tokamak physics.

\subsection{Data Acquisition and Signal Analysis}

The TALIF data acquisition system consists of an amplified photomultiplier tube (Hammamatsu H11526-20-NF) connected to a high-speed gated integrator and boxcar averager (Stanford Research Systems Model SRS250). The PMT amplifier is a $36 \mathrm{~dB}$ high-speed amplifier (Hammamatsu C5594), with an operating range of $50 \mathrm{kHz}$ to $1.5 \mathrm{GHz}$. Real-time background subtraction is used to suppress background fluctuations. A schematic of the background subtraction is shown in Figure 5.20. The boxcar acquires data at twice the laser repetition rate $(40 \mathrm{~Hz})$. Thus, signal and background measurements are taken sequentially. The pulses are integrated in time, with every other pulse inverted, then averaged together. This subtracts the background from the induced fluorescence. The averaged data, laser 
wavelength, measured from the $615 \mathrm{~nm}$ beam with a High Finesse WS7 wavemeter, and laser energy, measured with a Gentec energy meter, are written to a file for each measurement.

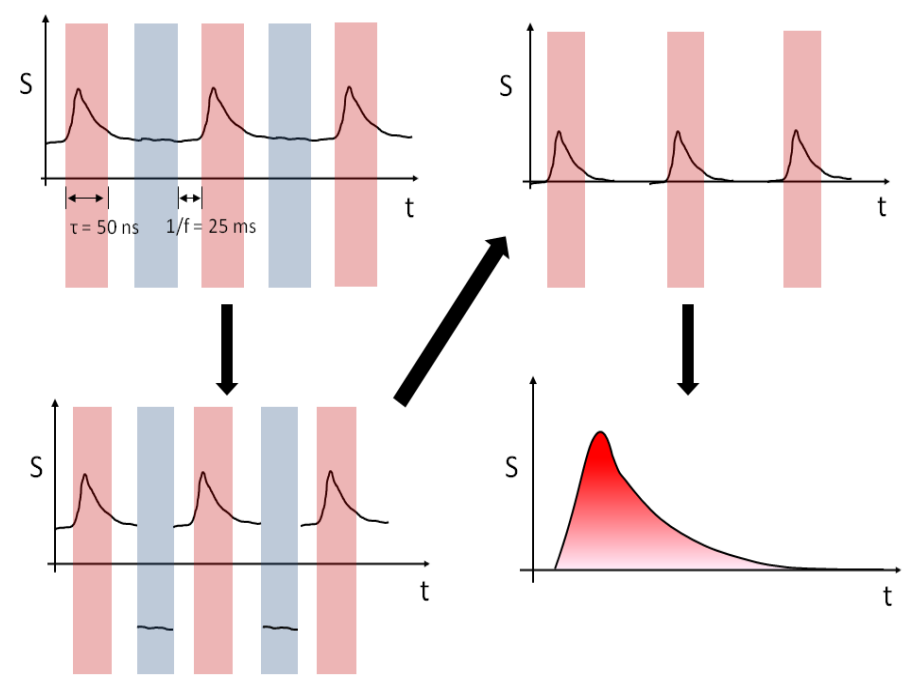

Figure 5.20: Steps in the signal processing procedure. The boxcar is gated at twice the laser repetition rate, recording both laser on and laser off signal. The laser off signal is inverted, subtracted from laser on signal, and then all the laser on signals are averaged together.

All TALIF signals were acquired using the same optical configuration, except that the narrow band filter in front of the detector was replaced for the different emission wavelengths of different species and the addition of up to two neutral density filters to prevent detector saturation, when necessary. The same gate width, $\sim 50 \mathrm{~ns}$, and the same number of shots were averaged for all measurements. Data were simultaneously recorded from the wavemeter, energy meter and boxcar averager, along with a time stamp for each data point. All three data records were recorded with a custom built LabWindows ${ }^{\mathrm{TM}}$ program.

Due to different recording rates, the three data records were not sampled simultaneously. The asynchronicity was corrected in post-processing. The wavelength data record time was used as a reference and the energy meter and the boxcar data were interpolated on that 
time scale using the IDL function INTERPOL with the "lsquadratic" keyword set. The INTERPOL function uses a least squares quadratic fit to each 4 point neighbors. Due to errors in the interpolation at the endpoints of the data records the first 10 and last 50 points were discarded before further analysis.

The energy corrected TALIF signals versus laser wavelength were fit to a Gaussian function. Because of comparable laser and absorption line widths for $\mathrm{Kr}$, a deconvolution of the two was necessary to accurately calculate the width of the $\mathrm{Kr}$ distribution. In general the measured lineshape for both $\mathrm{H}$ and $\mathrm{Kr}$ is a convolution of the Doppler broadened absorption lineshape and the lineshape of the laser photon pairs. The total lineshape is

$$
S(\nu)=\frac{\beta \sigma_{2 \gamma} E^{2}}{h \nu} \int d \tau_{1} \int d \tau_{2} \exp \left[\frac{-\left(\left(\nu-\tau_{1}\right)^{2}+\left(\nu-\tau_{2}\right)\right)^{2}}{2 \sigma_{l}^{2}} \exp \left[\frac{-\left(\nu_{0}-\tau_{1}-\tau_{2}\right)^{2}}{2 \sigma_{d}^{2}}\right]\right.
$$

where $\beta$ is a statistical factor, $\sigma_{2 \gamma}$ is the cross-section for two-photon absorption, $E$ is the laser energy, $h$ is Planck's constant, $\nu$ is the laser frequency, $\nu_{0}$ is the absorption frequency, and $\sigma_{l}$ and $\sigma_{d}$ are the laser line width and Doppler line width, respectively. Therefore, the measured lineshape has a width

$$
\sigma_{s}=\frac{1}{2} \sqrt{2 \sigma_{l}^{2}+\sigma_{d}^{2}}
$$

when plotted verses the 2-photon frequency $(102 \mathrm{~nm} / c)$. When plotted verses the 1-photon frequency, i.e., the wavelength of the incident light $(204 \mathrm{~nm} / c)$ the measured width becomes

$$
\sigma_{s}=\sqrt{2 \sigma_{l}^{2}+\sigma_{d}^{2}}
$$

Note the difference of a factor of $\frac{1}{2}$ between Eqns 5.16 and 5.17. For $\mathrm{H}, \sigma_{l} \ll \sigma_{d}$, and the 
measured width is

$$
\sigma_{s} \cong \frac{1}{2} \sigma_{d}
$$

For Kr, the Doppler width is obtained from the full form of Eqn (5.17) using the known laser line width and measured signal width,

$$
\sigma_{d}=\frac{1}{2} \sqrt{4 \sigma_{s}^{2}-\sigma_{l}^{2}}
$$

The neutral temperature is then calculated from the Doppler relationship,

$$
T=m c^{2}\left(\frac{\sigma_{d}}{\nu_{0}}\right)^{2} .
$$

The integral of the Gaussian representation of the neutral velocity distribution is calculated analytically from the width and the fit amplitude

$$
I_{\text {neutral }}=2 A_{0} \sqrt{\pi \frac{\sigma_{d}^{2}}{2}}
$$

where $A_{0}$ is the fit amplitude.

\subsection{Calibration}

In single-photon LIF measurements, Rayleigh scattering is used to calibrate the entire optical system. The LIF signal of the species under investigation is compared to the Rayleighscattered signal from a species with known Rayleigh cross-section. ${ }^{24}$ Rayleigh calibration is problematic because of line saturation and small signal levels. TALIF calibration is somewhat easier due to the ability of TALIF to directly probe the ground state of the target 
species. Simple comparison with the TALIF signal from a species of known density provides a calibration factor.

Neutral krypton is an excellent calibration species. There is a ground state transition, $4 p_{1}{ }^{6} S^{0} \rightarrow 5 p^{\prime}[3 / 2]_{2}$, at $102.1 \mathrm{~nm}$ (204.2 nm laser wavelength), that decays at $826 \mathrm{~nm}$. The absorption wavelength is very close to the hydrogen wavelength, thus the laser performance and characteristics are nearly identical for the two lines.

For accurate calibration, the laser energy (intensity) must be below the saturation level of the absorption line. If the energy is above the saturation level the absorption line will be artificially broadened, resulting in an inaccurate calculation of the neutral hydrogen density. The saturation level (energy) is determined by measuring the TALIF signal as a function of laser energy. Theoretically, the TALIF signal should scale as the laser energy squared, $S \sim E^{2}$, see Figure 5.21. The saturation energy in $\mathrm{Kr}$ was determined to be $\sim 200 \mu \mathrm{J}$ for a $25 \mathrm{~cm}$ focal length lens and $\sim 800 \mu \mathrm{J}$ for a $50 \mathrm{~cm}$ focal length lens. The increase in saturation energy is consistent with the increase in focal spot size between the two lenses. The minimum spot radius for a focused beam is

$$
r_{\min }=f \theta
$$

where $f$ is the focal length of the lens and $\theta$ is the divergence of the laser. Increasing the focal length by a factor of two increases the minimum spot radius by a factor of two and therefore the spot area by a factor of four. For constant laser energy the laser intensity decreases by a factor of four and a larger laser energy is required for saturation. 


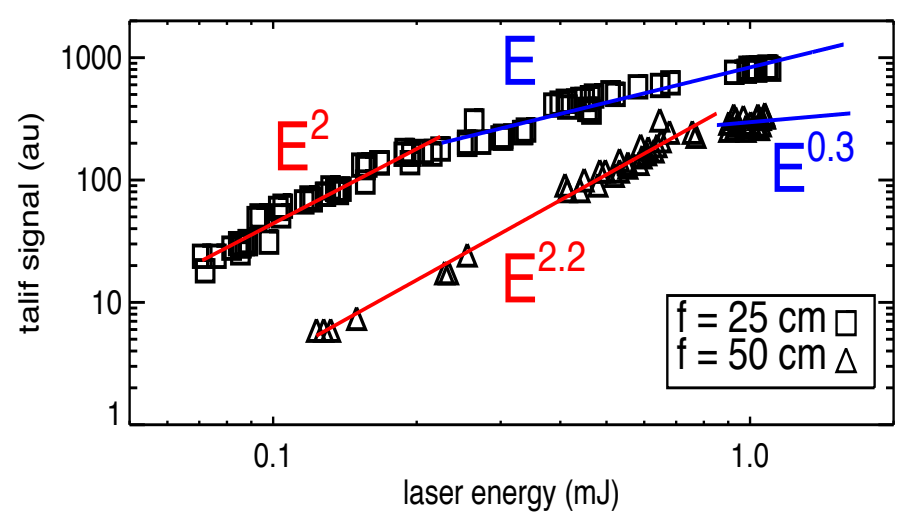

Figure 5.21: Krypton saturation energy measured for two different focal length lenses. Fits show the energy range in which the signal is unsaturated, $E^{2}$, and saturated, not $E^{2}$. Saturation energy increases with increase in focal spot size, as expected.

\subsubsection{Hydrogen Calibration}

Calibration is accomplished by measuring the number of photons emitted from a species of known density for a given laser intensity with the number of photons from a species of unknown density for a given laser intensity.

$$
\frac{S_{A}\left(I_{\text {las }}\right)}{n_{A}}=\frac{S_{X}\left(I_{l a s}\right)}{n_{X}}
$$

where $A$ and $X$ are species of known and unknown density respectively and $I_{l a s}$ is the laser intensity associated with a measured signal. The TALIF signal measured by a photomultiplier tube (PMT) detector is

$$
S_{P M T}=n \sigma^{(2)}\left(\frac{I}{\nu}\right)^{2} A \tau T \xi \frac{\Delta \Omega}{4 \pi} G
$$

where $n$ is the density of the species, $\sigma^{(2)}$ is the cross-section for two-photon absorption, $\nu$ is the absorption frequency, $A$ is the Einstein coefficient for spontaneous emission, $\tau$ is the 
state lifetime, $T$ is the optical transmission of the system, $\xi$ is the quantum efficiency of the detector, $G$ is the detector gain and $\frac{\Delta \Omega}{4 \pi}$ is the solid angle of collection. Taking the ratio of the signals in $\mathrm{H}$ and a Kr fill at known pressure (density),

$$
\frac{S_{K r}}{S_{H}}=\frac{n_{K r} \sigma_{K r}^{(2)}\left(\frac{I_{K r}}{\nu_{K r}}\right)^{2} A_{K r} \tau_{K r} \xi_{K r} G_{K r}}{n_{H} \sigma_{H}^{(2)}\left(\frac{I_{H}}{\nu_{H}}\right)^{2} A_{H} \tau_{H} T_{H} \xi_{H} G_{H}}
$$

where the solid angle have cancelled as the collection system is the same for both species. Solving for $n_{H}$

$$
n_{H}=n_{K r} \frac{S_{H}}{S_{K r}}\left(\frac{I_{K r} \nu_{H}}{I_{H} \nu_{K r}}\right)^{2} \frac{A_{K r}}{A_{H}} \frac{\tau_{K r}}{\tau_{H}} \frac{T_{K r}}{T_{H}} \frac{\xi_{K r}}{\xi_{H}} \frac{G_{K r}}{G_{H}}
$$

From this equation a calibration factor is defined as

$$
c f=\frac{S_{K r}}{n_{K r} I_{K r}^{2} G_{K r} T_{K r}} .
$$

Using this calibration factor, the absolute density of ground state hydrogen is calculated from the measured TALIF signal through

$$
n_{H}=\frac{S_{H}}{I_{H}^{2} G_{H} T_{H}} \frac{1}{c f} .
$$

\subsubsection{Deuterium Calibration}

The cross-section for two-photon absorption is

$$
\sigma_{2 \gamma}=\frac{\omega_{0} \pi e^{4}}{2 c^{2} \epsilon_{0}^{2} \hbar^{3}}|M|^{2} g(\Delta \nu) G
$$


where $\omega_{0}$ is the laser frequency, $g(\Delta \nu)$ is a lineshape function, $G$ is a statistical factor and $M_{f i}$ is the matrix element comprised of the wave functions for the lower and excited states. The difference between the wave functions for hydrogen and deuterium is insignificant, thus the cross-section for two-photon absorption is unchanged and the calibration factor applies to both hydrogenic species.

\subsection{Signal-to-Noise}

For the TALIF diagnostic to be effective, either on a small helicon or a fusion device, a sufficient signal-to-noise ratio (SNR) must be achieved. The SNR can be calculated for expected conditions in both systems. Additionally, this calculation provides a lower bound on measured density levels for given laser and plasma parameters.

The pumping rate from the $n=1$ to the $n=3$ level is

$$
\frac{d N_{3}}{d t}=\frac{\beta N_{1} \sigma_{2 \gamma} I_{1} I_{2}}{\hbar\left(\omega_{1} \omega_{2}\right)^{1 / 2}}
$$

where $\beta$ is beam correlation factor, 1 for single beam, 4 for two counter propagating beams, $N_{1}$ is the density of state $1, \omega_{1}, I_{1}, \omega_{2}$ and $I_{2}$ are the frequencies and intensities of beams one and two respectively, and $\sigma_{2 \gamma}$ is the cross-section for two-photon absorption,

$$
\sigma_{2 \gamma}=\frac{\omega_{0} \pi e^{4}}{2 c^{2} \epsilon_{0}^{2} \hbar^{3}}|M|^{2} g(\Delta \nu) G,
$$

where $M$ is the transition matrix element and $G$ is a statistical factor, 2 for a co-linear

beam, 1 for counter propagating beams, and $g(\Delta \nu)$ is a lineshape function. ${ }^{25}$ The lineshape function is a convolution of the one-photon laser lineshape and the absorption lineshape for 
the two-photon resonance, ${ }^{11}$

$$
g\left(\Delta \nu=2 \nu_{L}-\nu_{A}\right)=g_{A}\left(2 \nu-\nu_{A}\right) * g_{L}\left(\nu-\nu_{L}\right) * g_{L}\left(\nu-\nu_{L}\right)
$$

where $g_{A}$ is the absorption lineshape, $g_{L}$ is the laser lineshape, $\nu_{A}$ is the two-photon absorption frequency, and $\nu_{L}$ is the laser frequency.

For a single beam, $\omega_{1}=\omega_{2}=\omega$, in the limit that the Doppler width of the hydrogen absorption line is much larger than the laser line width, $\omega_{l}$, whenever the laser frequency is equal to the two-photon resonance frequency $g(\Delta \nu=0)$ is

$$
g(\Delta \nu=0)=\left[\frac{4 \ln (2)}{\pi}\right]^{1 / 2} \frac{1}{\left(\Delta \omega_{D}^{2}+2 \Delta \omega_{l}^{2}\right)^{1 / 2}}
$$

For counter-propagating beams the Doppler width goes to 0 and $g(\Delta \nu=0)$ is

$$
g(\Delta \nu=0)=\left[\frac{4 \ln (2)}{\pi}\right]^{1 / 2} \frac{1}{\sqrt{2} \Delta \omega_{l}} .
$$

Using values for $\sigma_{2 \gamma} / g(\Delta \nu) G$ calculated by Tung et al. ${ }^{26}$, the pumping rate for the $1 s \rightarrow$ $3 d$ Doppler broadened and $1 s \rightarrow 3 s$ Doppler-free transitions are calculated. For the $1 s \rightarrow$ $3 d$ transition, assuming a single beam with energy of $8.5 \mathrm{~mJ}$ per pulse focused to a $0.1 \mathrm{~mm}$ spot,

$$
\begin{aligned}
\frac{d N_{3}}{d t} & =\frac{\beta G \sigma_{2 \gamma} g(\Delta \nu) I^{2}}{h \omega} n_{0} \\
& =\frac{1 \times 2 \times 18.3 \times 10^{-18}}{1.2 \times 10^{-17}} \sqrt{\frac{4 \ln (2)}{\pi}} \frac{1}{\left(\Delta \omega_{d}^{2}+2 \Delta \omega_{l}^{2}\right)^{1 / 2}} I^{2} n_{0} \\
& \approx 5.4 \times 10^{7} T^{-1 / 2} n_{0},
\end{aligned}
$$


where $T$ is the neutral temperature in $\mathrm{eV}$. This expression has units of $\mathrm{cm}^{-3} \mathrm{~s}^{-1}$ if the density has units of $\mathrm{cm}^{-3}$.

For the $1 s \rightarrow 3 s$ transition, assuming an $8 \mathrm{~mJ}$ per pulse beam split into two $4 \mathrm{~mJ}$ per pulse beams

$$
\begin{aligned}
\frac{d N_{3}}{d t} & =\frac{\beta G \sigma_{2 \gamma} g(\Delta \nu) I^{2}}{h \omega} n_{0} \\
& =\frac{2 \times 2 \times 2.42 \times 10^{-18}}{1.2 \times 10^{-17}} \sqrt{\frac{4 \ln (2)}{\pi}} \frac{1}{\sqrt{2} \Delta \omega_{l}} I^{2} n_{0} \\
& \approx 3.4 \times 10^{8} n_{0},
\end{aligned}
$$

where again the units are $\mathrm{cm}^{-3} \mathrm{~s}^{-1}$ if the density has units of $\mathrm{cm}^{-3}$.

The measured signal is related to the pumping rate by

$$
S=\gamma \frac{\Omega}{4 \pi} \pi r^{2} L \frac{d N_{3}}{d t},
$$

with $\gamma$ the branching ratio for the observed decay path, $\Omega$ the solid angle of collection, and $\pi r^{2} L$ the emission volume. Using approximate design values for installation on the DIII-D tokamak, which are similar to the values for these experiments, the solid angle of collection and emission volume to be $0.05 \mathrm{str}$ and $\sim 3 \times 10^{-4} \mathrm{~cm}^{3}$, respectively, the calculated signal is

$$
S \approx 4 \times 10^{-7} \frac{d N_{3}}{d t}
$$

The background in the DIII-D tokamak was estimated from the two-fluid edge neutral code UEDGE. The code models the electron density and temperature and neutral density, which are then used to calculate the background emissivity at $656 \mathrm{~nm}$, which is then inte- 
grated along a chord, using the same optical characteristics given above, to calculate the background emission. From this code the background is estimated to be $\sim 10^{14}$ photon/s. ${ }^{27}$ If the background is dominated by Poisson noise on the laser timescale (10 ns), that is the variation in the background is $\sqrt{N}$, where $N$ is the number of counts, then the minimum resolvable density is determined by

$$
S>\sqrt{10^{14}}
$$

For the $1 s \rightarrow 3 d$ transition, assuming a neutral temperature of $100 \mathrm{eV}$ for a tokamak plasma, the minimum measurable neutral density is determined from

$$
S=4 \times 10^{-7} \times 5.4 \times 10^{7} \times(100)^{-1 / 2} \times n_{0}>10^{7}
$$

The minimum density that satisfies the above relation is $n_{0}>10^{13} \mathrm{~m}^{-3}$. As shown in Eqn 5.36, the signal for the $1 s \rightarrow 3 s$ transition is independent of neutral temperature. Eqn 5.40 becomes

$$
S=4 \times 10^{-7} \times 3.4 \times 10^{8} \times n_{0}>10^{7} .
$$

For the DIII-D background the minimum resolvable density drops to $\sim 10^{11} \mathrm{~m}^{-3}$. However, these calculations assume the same beam radius for both schemes. It is likely that the Doppler-free beam will be unfocused, therefore the a two-order of magnitude gain in detection sensitivity is unlikely.

In a helicon plasma the background is much smaller. Assuming a background of $10^{10}$ photons/s, as was calculated for the Oak Ridge National Laboratory Physics Integration eXperiment (PhIX) helicon source, ${ }^{28}$ and a neutral temperature of $1 \mathrm{eV}$, the minimum resolvable density is $10^{11} \mathrm{~m}^{-3}$ for the $1 s \rightarrow 3 d$ transition and a minimum resolvable density 
of $10^{10} \mathrm{~m}^{-3}$ for the $1 s \rightarrow 3 s$ transition, again assuming the same laser intensities for both schemes. 


\section{References}

[1] M. Göeppert-Mayer, "Über elementarakte mit zwei quantenspruengen," Annalen der Physik, vol. 401, pp. 273-294, 1931.

[2] C. Seiser, Measurement of two-photon absorption laser-induced Lyman- $\alpha$ fluorescence: A diagnostic for atomic number densities of hydrogen isotopes in magnetically confined fusion plasmas. PhD thesis, Technischen Universitaet Berlin, 1998.

[3] W. Kaiser and C. G. B. Garrett, "Two-photon excitation in caf 2 : eu ${ }^{2+}, "$ Physical Review Letters, vol. 7, pp. 229-231, 1961.

[4] T. W. Haensch, S. A. Lee, R. Wallenstein, and C. Wieman, "Doppler-free two-photon spectroscopy of hydrogen 1s - 2s*," Physical Review Letters, vol. 34, pp. 307-309, 1975.

[5] J. Bokor, R. R. Freeman, J. C. White, and R. H. Storz, "Two-photon excitation of the n=3 level in h and d atoms," Physical Review A, vol. 24, pp. 612-614, 1981.

[6] T. Kajiwara, K. Takeda, K. Muraoka, T. Okada, M. Maeda, and M. Akazaki, "Coaxial laser fluorescence system by two-photon excitation for atomic hydrogen detection in high-temperature plasmas," Japanese Journal of Applied Physics, vol. 29, pp. L826L828, 1990.

[7] D. Voslamber and J. Seidel, "Measurement of the deuterium/tritium fuel mixture in magnetic confinement fusion plasmas by doppler-free two-photon spectroscopy," Review of Scientific Instruments, vol. 70, p. 928931, 1999.

[8] D. Voslamber, "Doppler-free two-photon excitation of lyman- $\alpha$ fluorescence for the di- 
agnostics of magnetically confined fusion plasmas," Review of Scientific Instruments, vol. 71, pp. 2334-2352, 2000.

[9] T. Kajiwara, K. Takeda, H. J. Kim, W. Z. Park, T. Okada, M. Maeda, K. Muraoka, and M. Akazaki, "Application of laser fluorescence spectroscopy by two-photon excitation into atomic density measurement in reactive plasmas," Japanese Journal of Applied Physics, vol. 29, pp. L154-L156, 1990.

[10] L. Chérigier, U. Czarnetzki, D. Luggenhölscher, V. S. von der Gathen, and H. F. Döbele, "Absolute atomic hydrogen densities in a radio frequency discharge measured by twophoton laser induced fluorescence imaging," Journal of Applied Physics, vol. 85, pp. 696$702,1999$.

[11] K. Niemi, V. S. von der Gathen, and H. F. Doebele, "Absolute calibration of atomic density measurements by laser-induced fluorescence spectroscopy with two-photon excitation," Journal of Physics D: Applied Physics, vol. 34, pp. 2330-2335, 2001.

[12] M. G. H. Boogaarts, S. Mazouffre, H. W. P. v. d. H. G. J. Brinkman, P. Vankan, J. A. M. can der Mullen, and D. C. Schram, "Quantitative two-photon laser-induced fluorescence measurements of atomic hydrogen densities, temperatures, and velocities in an expanding thermal plasma," Review of Scientific Instruments, vol. 73, pp. 73-86, 2002.

[13] J. Jolly and J. P. Booth, "Atomic hydrogen densities in capacitively coupled very highfrequency plasmas in $\mathrm{h}_{2}$ : Effect of excitation frequency," Journal of Applied Physics, vol. 97, p. 103305, 2005.

[14] W. Demtröder, Laser Spectroscopy. Springer-Verlag, 2008. 
[15] P. A. Keiter, Experimental Investigation of Ion Temperature Anisotropy Driven Instabilities in a High Beta Plasma. PhD thesis, West Virginia University, 1999.

[16] H.-J. Kunze, Introduction to Plasma Spectroscopy. Springer-Verlag, 2009.

[17] H. R. Griem, Principles of Plasma Spectroscopy. Cambridge University Press, 1997.

[18] R. Shankar, Principles of Quantum Mechanics. Springer, 1994.

[19] J. P. Booth, J. Derouard, M. Fadlallah, L. Cabaret, and J. Pinard, "Electric field measurements in discharges by doppler-free two-photon laser stark spectroscopy of atomic hydrogen," Optics Communications, vol. 132, pp. 363-370, 1996.

[20] M. J. Goeckner and J. Goree, "Laser induced fluorescence measurement of plasma ion temperatures: Corrections for power saturation," Journal of Vacuum Science $\mathbb{E}$ Technology, A: Vacuum, Surfaces, and Films, vol. 7, pp. 977-981, 1989.

[21] M. J. Goeckner, J. Goree, and T. E. Sheridan, "Saturation broadening of laser-induced fluorescence from plasma ions," Review of Scientific Instruments, vol. 64, pp. 996-1000, 1993.

[22] R. W. Boyd, Nonlinear Optics. Academic Press, 2008.

[23] C. C. Davis, Laser and Electro-Optics: Fundamentals and Engineering. Cambridge University Press, 2000.

[24] H. F. Döbele, U. Czarnetzki, and A. Goehlich, "Diagnostics of atoms by laser spectroscopic methods in plasmas and plasma-wall interaction studies (vacuum ultraviolet and two-photon techniques)," Plasma Sources Science and Technology, vol. 9, pp. 477-491, 2000. 
[25] U. Czarnetzki, K. Miyazaki, T. Kajiwara, K. Muraoka, M. Maeda, and H. F. Döbele, "Comparison of various two-photon excitation schemes for laser-induced fluorescence spectroscopy in atomic hydrogen," Journal of the Optical Society of America B: Optical Physics, vol. 11, pp. 2155-2162, 1994.

[26] J. H. Tung, A. Z. Tang, G. J. Salamo, and F. T. Chan, "Two-photon absorption of atomic hydrogen from two light beams," Journal of the Optical Society of America B: Optical Physics, vol. 3, pp. 837-848, 1986.

[27] R. M. Magee, M. E. Galante, and E. E. Scime, "A proposal for two-photon absorption laser-induced fluorescence measurements of edge neutral density in diii-d," tech. rep., West Virginia University, 2012.

[28] R. M. Magee, "Estimate of talif signal-to-background level for phix," tech. rep., West Virginia University, 2012. 


\section{Chapter 6}

\section{Steady-State Plasma TALIF}

\section{Measurements}

Neutral and plasma densities in hydrogen, deuterium and krypton were measured for varying source parameters, rf power, magnetic field, pressure and driving frequency. For low power operation, $<1 \mathrm{~kW}$, krypton and hydrogen (deuterium) displayed very different behaviors. As will be outlined later, much higher plasma densities were achieved in krypton than in hydrogen for similar discharge parameters. Visually, the plasmas look very different, with hydrogen (deuterium) almost uniform in color and intensity throughout, while krypton displays significant core structure in the center of the discharge, indicative of the helicon operating mode (see Figure 6.1.) 

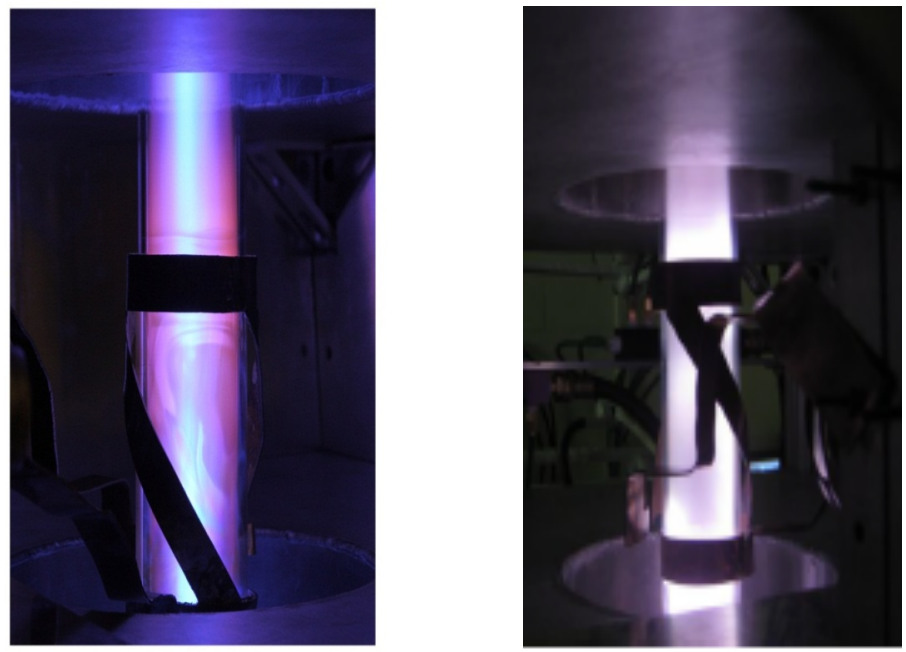

Figure 6.1: Krypton plasma (left) in helicon mode and hydrogen plasma (right) for similar operating parameters. Helicon mode was not achievable in hydrogen, possibly due to the added sink for energy absorption presented by molecular dissociation.

\subsection{Krypton}

In addition to being the calibration species, krypton also provided a means to directly study ground state density in a monatomic helicon plasma. Lower hybrid resonance effects and neutral depletion were studied, phenomena that had previously been measured using indirect methods. Additionally, the behavior of neutrals in the different helicon operating modes, outlined in Chapter 2, was investigated.

\subsubsection{Parametric Scans in Krypton Plasma}

Helicon sources are characterized by high density and high ionization fraction. This high ionization fraction, approaching 100\%, was first proposed by Boswell to explain pressure balance between the hot plasma and cold neutral gas in the center of the discharge. ${ }^{1}$ However, 
he had no method to directly measure the ionization fraction. In these experiments, the local ionization fraction is directly measured by coupling TALIF measurements of Kr neutrals and Langmuir probe measurements of electrons.

As rf power was increased, krypton neutral density decreased and plasma density simultaneously increased, see Figure 6.2. Such behavior is expected as neutrals are fuel for the plasma. Increasing plasma density, for constant fill pressure, must result in decreasing neutral density. The discrete jump in plasma density above $200 \mathrm{~W}$ is consistent with previous measurements in helicon sources ${ }^{2}$ and can be interpreted as switching between the inductive and helicon operating modes. Whenever the source is operated in helicon mode the neutral density begins to decrease rapidly with increasing power. For powers above 700 W, the neutral density in the core is less than the local plasma density, giving an ionization fraction approaching 100\%. Similar behavior was observed when increasing magnetic field

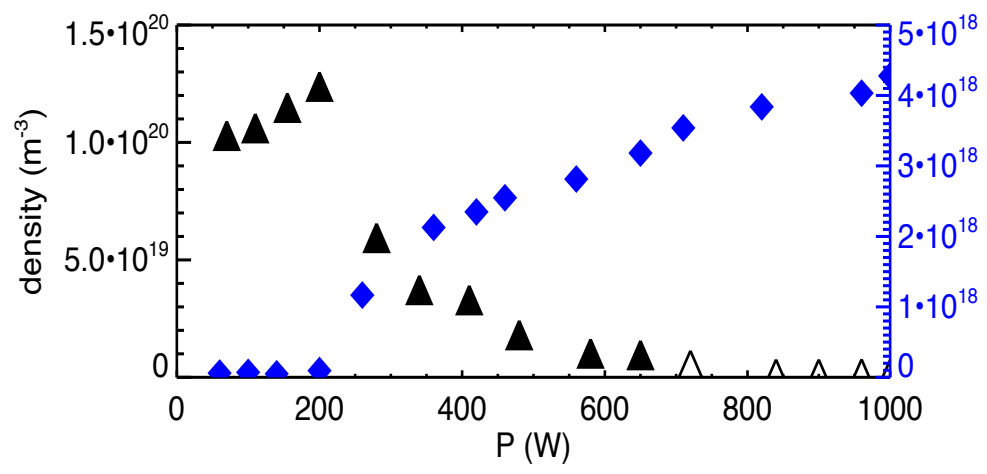

Figure 6.2: Neutral Kr (black triangles, left axis) and electron (blue diamonds, right axis) density as a function of rf power. Open symbols correspond to neutral density values less than $2 \times 10^{18} \mathrm{~m}^{-3}$.

at constant rf power, see Figure 6.3. Below $600 \mathrm{G}$ both plasma and neutral densities were constant. Above this field strength the plasma density begins to rise, nearly linearly with field strength, and the neutral density drops. The increase in plasma density with magnetic field strength is consistent with the helicon mode of operation, however the magnitude of 
the density is about a factor of 10 lower than that predicted by the simple helicon dispersion relation, see Figure 6.4. As plasma is created primarily in the source region, then diffuses into the expansion chamber, with an areal expansion ratio of $\sim 10$, it is reasonable that the plasma density in the source is $\sim 10 \mathrm{x}$ that measured in the expansion, and the source satisfies the helicon dispersion relation (see Figure 6.4.) Only for magnetic field strengths above $600 \mathrm{G}$, after the transition to helicon mode, did the corrected density satisfy the helicon dispersion relation. Thus, at low field the source is not operated in helicon mode.

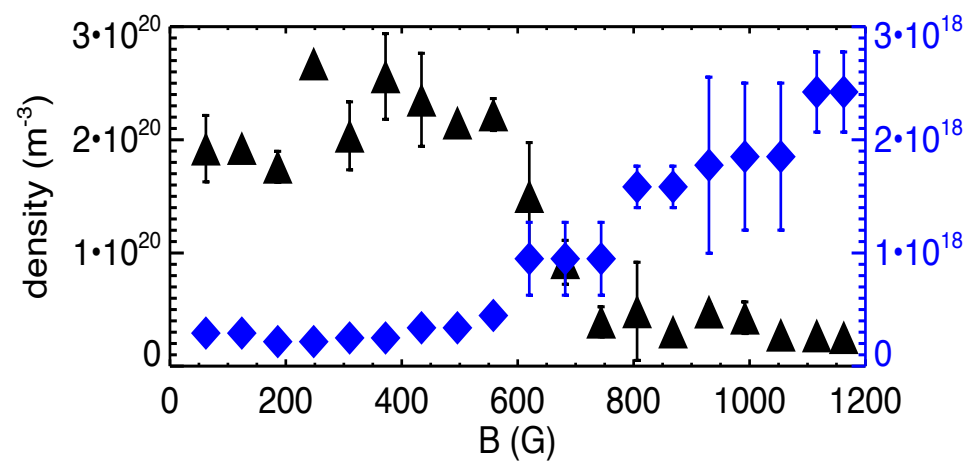

Figure 6.3: Neutral Kr (black triangle, left axis) and electron (blue diamonds, right axis) density as a function of source magnetic field.

Between 600 and $700 \mathrm{G}$, the neutral density drops by nearly an order of magnitude, despite only a 2-3x increase in the plasma density. At these field strengths, the plasma density is over an order of magnitude lower than the neutral density. Thus, the drop in neutral density cannot be explained solely by the increase in plasma density, indicating some additional mechanism is responsible for depleting neutrals in the core.

One possible explanation for neutral depletion is neutral heating. As the neutral density in the core of the plasma drops, neutrals are heated to maintain pressure balance between the neutrals and plasma. The line width of the $205 \mathrm{~nm}$ laser is smaller than the Doppler width of a neutral Kr atom in plasma, thus the neutral temperature can be directly measured. As 


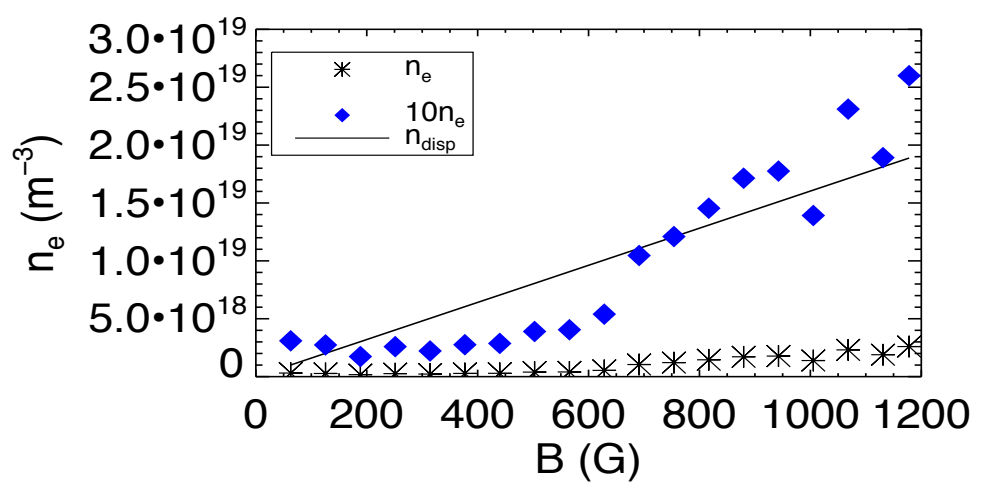

Figure 6.4: Helicon dispersion relation (solid) and plasma density in $\mathrm{Kr}$, measured in the expansion region with an rf compensated Langmuir probe. Stars are measured density, triangles are 10x measured density, the expected density in the source region. Solid line in the predicted density from the simple helicon dispersion relation, Eqn 2.4.

the plasma transitions into a high density operating mode and the neutral density drops, the neutral temperature decreases, rather than increases, see Figure 6.5. Thus, the observed neutral depletion is not caused by neutral heating.

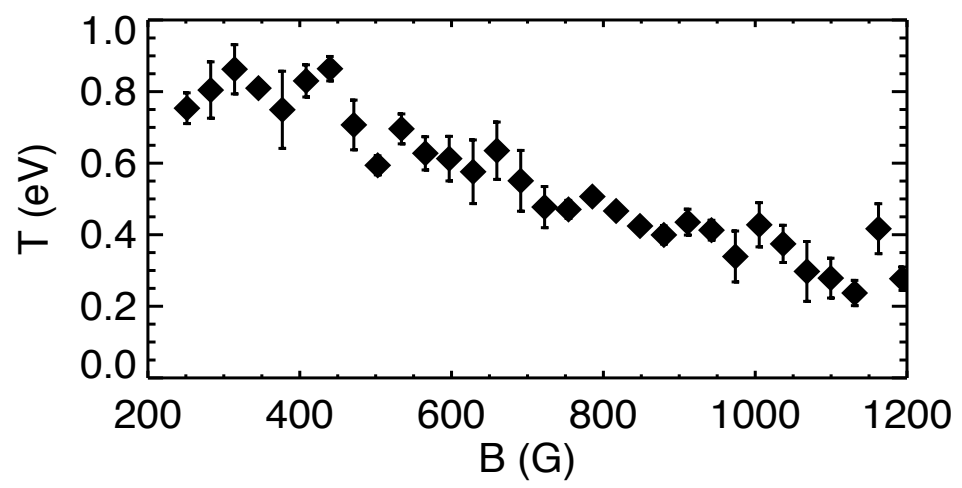

Figure 6.5: Neutral Kr temperature as a function of applied magnetic field strength. The temperature decreases as the plasma density increases, eliminating neutral heating as a mechanism for neutral depletion.

The other possible cause for neutral depletion is ion or neutral pumping, which was described in Chapter 2. Two different gas fill configurations were used, flowing and static, to further explore the neutral depletion process. In the flowing configuration, gas is continuously 
added and removed from the chamber, replenishing the fuel source for the plasma. In the static configuration gas is added to an unpumped chamber up to a certain fill pressure then valved off so that no gas can be added to or leave the chamber. Only krypton was run in this configuration as hydrogen plasmas were too unstable in a static fill. The krypton plasma behavior is essentially the same between the two configurations, i.e. increasing plasma density with field strength. However, the neutral density exhibits very different behavior. In the flowing configuration, shown in Figure 6.3, the neutral density decreases by more than a factor of ten between 500 and 1000 G, while in the static configuration, the neutral density is roughly constant with with field. This suggests that the neutral pumping mechanism is actually the result of neutrals being mechanically pumped out of the chamber.

In the static configuration, the plasma density is higher than in a flowing configuration. A similar increase in plasma density has been previously observed in a static helium helicon plasma. ${ }^{3}$ However they observed enhanced neutral depletion in the static case, whereas here, neutral depletion does not occur in the static configuration. The helium measurements are fundamentally different than those presented here. They used a metastable LIF scheme, which is highly sensitive to electron temperature and density, and used a collisional radiative model to calculate the ground state neutral density. While those measurements were indirect and made use of a model, these are direct ground state neutral density measurements, eliminating the need for a model and the assumptions associated it.

The neutral and plasma density profiles in krypton also reveal significant neutral depletion when operating in flowing helicon mode, see Figure 6.7. In an inductively coupled mode the plasma density is low, $\sim 10^{17} \mathrm{~m}^{-3}$, and both neutral and plasma density profiles are flat over much of the chamber radius. In helicon mode the plasma density is $\sim 40 \times$ larger in the core of the plasma, $\sim 4 \times 10^{18} \mathrm{~m}^{-3}$, and the plasma density profile is very centrally peaked. The 


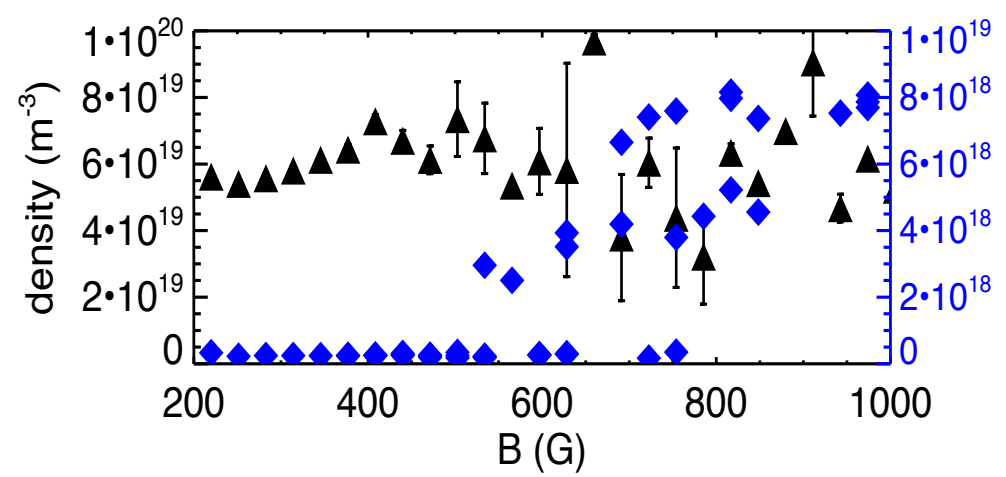

Figure 6.6: Neutral Kr (black triangles, left axis) and electron (blue diamonds, right axis) density as a function of source magnetic field for static gas fill.

neutral density profile is hollow, with the smallest density in the center, where the plasma density is largest, and increases by almost a factor of 20 between the center and edge.

When the driving frequency is varied, while the rf power and magnetic field are held constant, the neutral density drops sharply when the plasma density increases sharply, see Figure 6.9. From both the scan of magnetic field strength (Figure 6.8) and driving frequency, it can be seen that plasma production is maximized as the antenna frequency approaches the lower hybrid resonance.

Due to the high krypton ion mass, the lower hybrid resonance was not accessible with the rf generation system used in these experiments. For a magnetic field strength of $600 \mathrm{G}$, the LH resonance is $\sim 4.3 \mathrm{MHz}$. The $\mathrm{rf}$ generator can output at this frequency, but the capacitance of the matching network is insufficient to efficiently match the plasma. The closest the driving frequency can get to the LH resonance is $\sim 2.8 \omega_{L H}$. However, the trend is still clear. When approaching the lower hybrid resonance plasma density increases. Similar behavior has previously been reported, both theoretically ${ }^{4}$ and experimentally. ${ }^{5}$

The krypton ionization fraction in a helicon can approach $100 \%$ in the center of the discharge, shown in Figure 6.10, even though the average plasma density is only $1 \%$ of the 

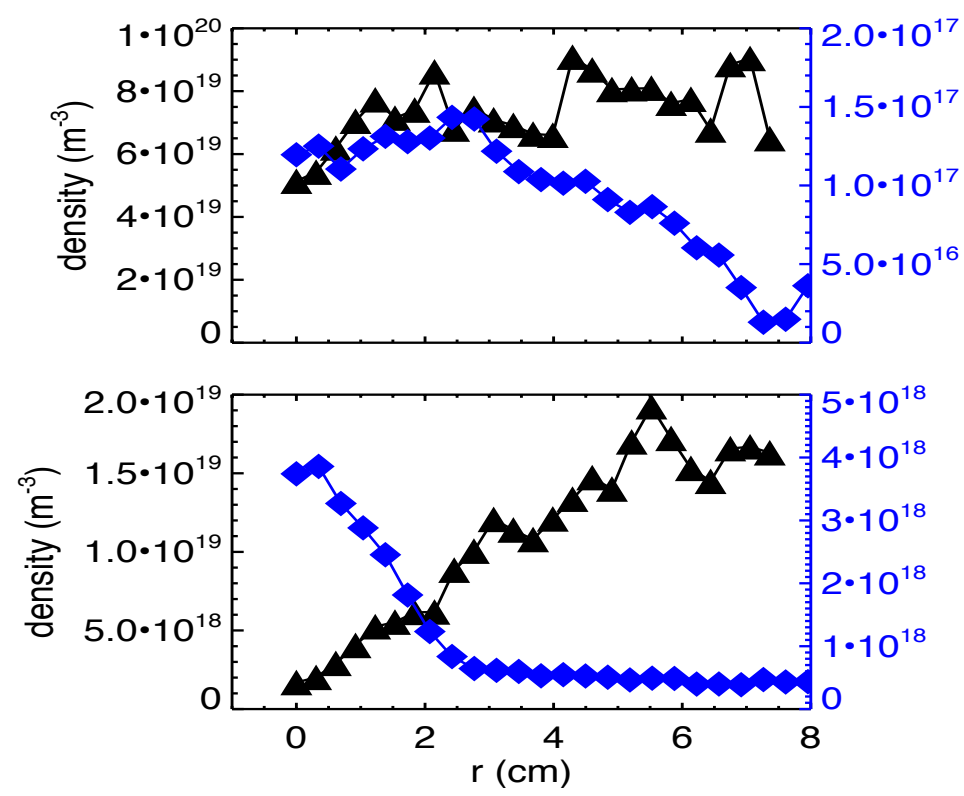

Figure 6.7: Radial neutral Kr (black triangles, left axis) and electron (blue angles, right axis) density in inductively coupled (top) and helicon (bottom) operating modes.

fill density. This is because locally, the neutral density is depleted to such a level that the plasma is fully ionized, confirming Boswell's hypothesis. The neutral depletion effect places an effective density limit on helicon sources, as above certain plasma densities the plasma effectively screens out neutrals from the high density region of the plasma. The lack of neutral fuel in the core prevents further increases in plasma density. Miljak and Chen have proposed that the density limit in a helicon is $\sim 10^{20} \mathrm{~m}^{-3} \cdot{ }^{6}$ This limit is discussed further in the next section.

\subsubsection{Axial Profiles in Krypton Plasma}

All measurements presented in the previous section were made in the expansion chamber, well away from the source region. Plasma is not created in this region, rather it diffuses into it along the magnetic field lines. To investigate the behavior of neutrals in the source region, 


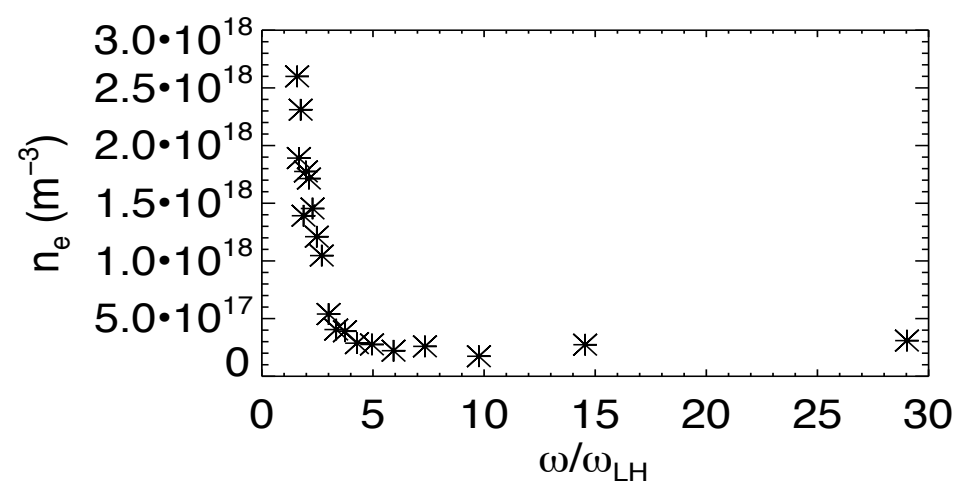

Figure 6.8: Kr plasma density as a function of the local lower hybrid resonance. Scanning the magnetic field scanned the lower hybrid resonance. Plasma production was maximized as the lower hybrid frequency in the plasma approached the driving frequency, held fixed at $13 \mathrm{MHz}$ in these experiments.

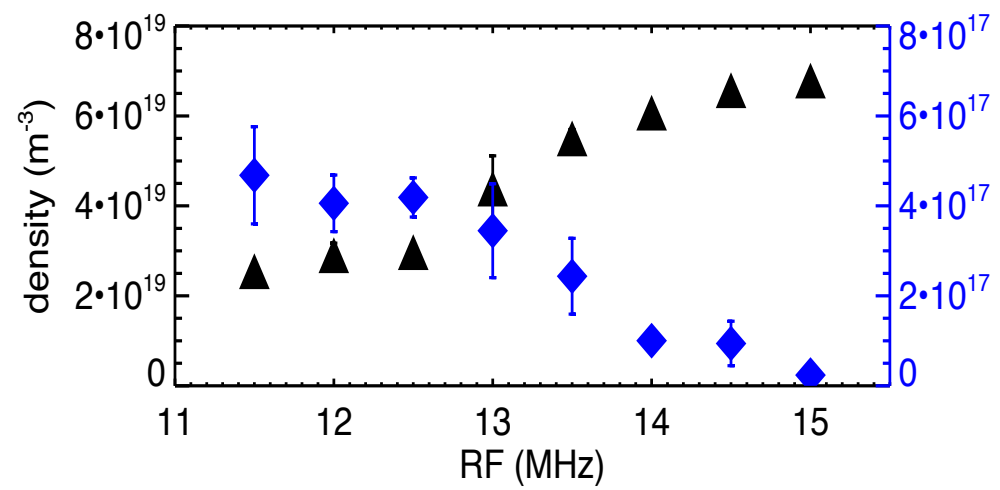

Figure 6.9: Radial neutral Kr (black triangles, left axis) and electron (blue diamonds, right axis) density as a function of driving frequency.

axial measurements were obtained. Due to alignment issues it was determined that the best method for making such measurements was to re-align the chamber with respect to the laser system, rather than steering the laser beam. The re-aligned chamber is shown in Figure 6.11. While high precision neutral density measurements were made in the source region, no plasma density measurements were made due to lack of diagnostic access. It is assumed that the plasma density is larger in the source region, perhaps by as much as a factor of 10, based on previous plasma density measurements in the source region of an argon helicon plasma. ${ }^{7}$ 


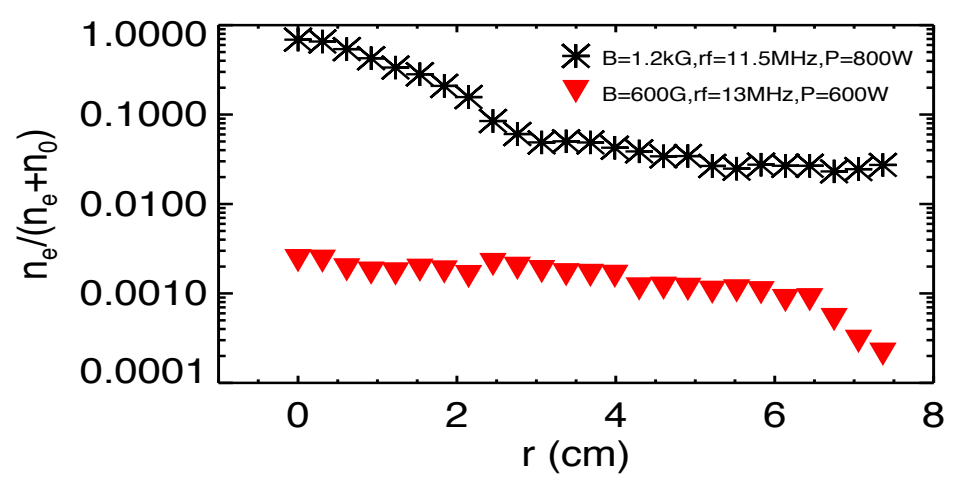

Figure 6.10: Radial Kr ionization fraction in helicon (black stars) and inductive (red triangles) modes.

With increasing rf power the axial neutral Kr density becomes increasingly depleted, so much so that above $700 \mathrm{~W}$ the neutral Kr density is below measurable levels (see Figure 6.12.) Above $500 \mathrm{~W}$ the magnitude of the neutral density decreases below the local electron density and the source approaches $100 \%$ ionization. Further increases in power do not result in increased plasma density. However, the plasma extent increases. As the plasma extends axially, filling more of the chamber a "neutral hole" of $100 \%$ ionized plasma is created. This explains the rapid drop off in the neutral density between the top of the chamber, $z=0$, and $z=13 \mathrm{~cm}$ at $900 \mathrm{~W}$ (Figure 6.12.) 


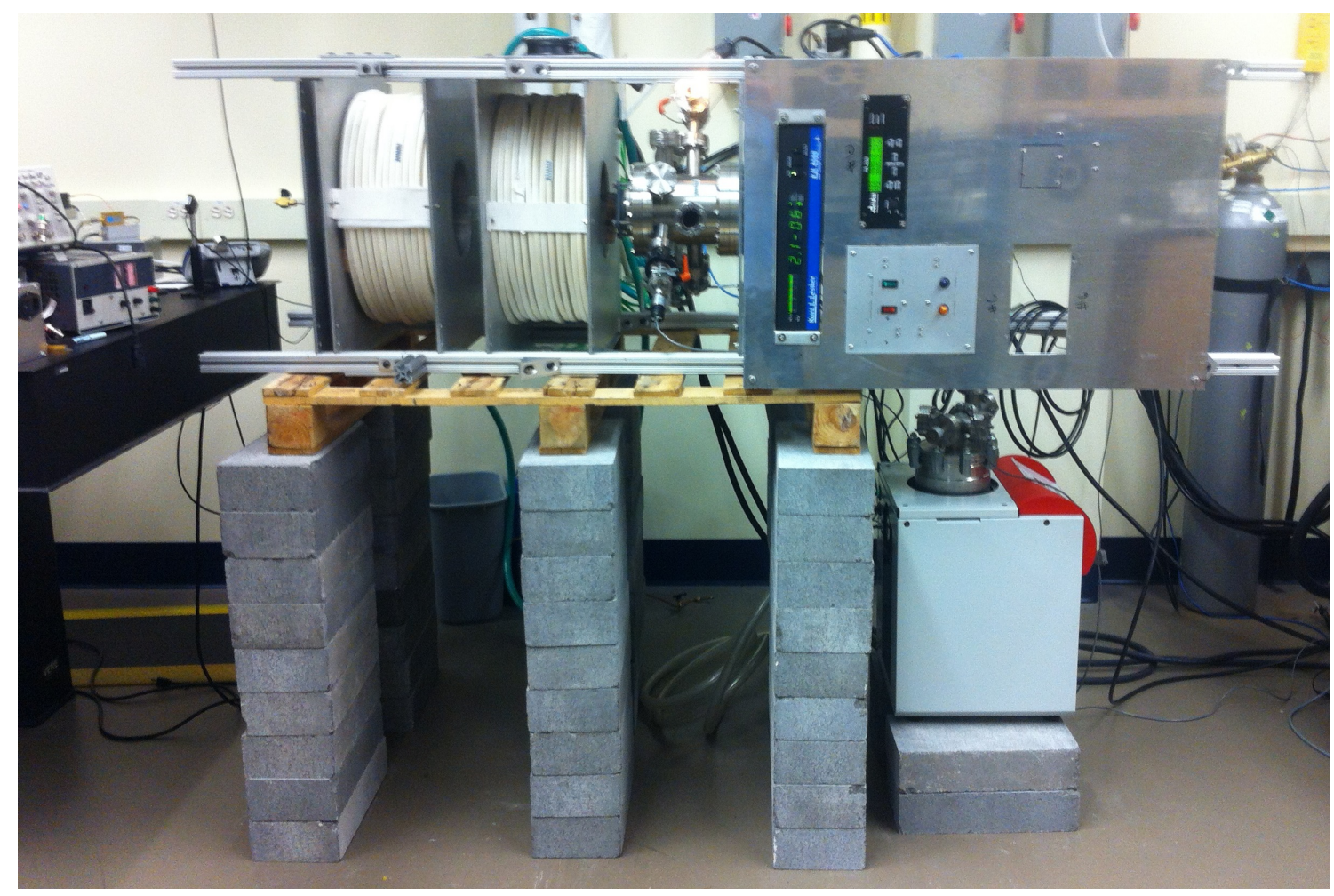

Figure 6.11: CHEWIE re-aligned for axial injection.

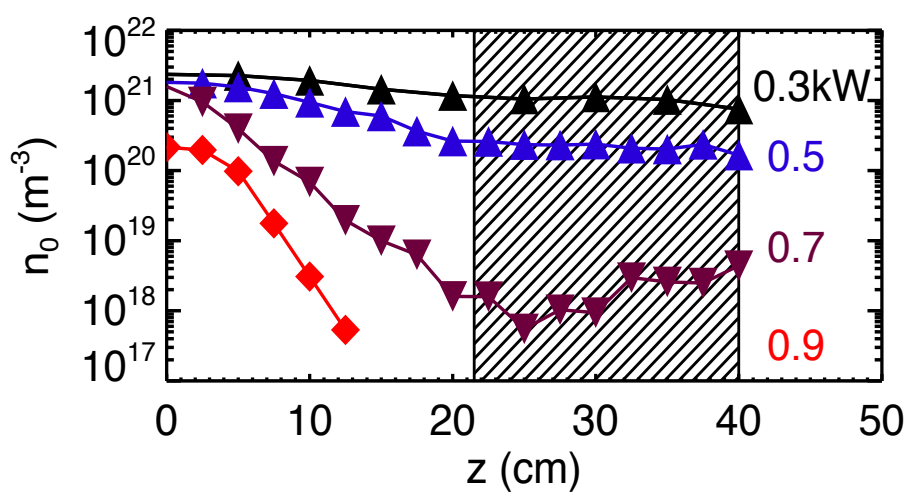

Figure 6.12: CW axial neutral krypton density. Cross-hatches indicate antenna region. The bottom loop of the antenna, closest to the turbo pump, is at $40 \mathrm{~cm}^{8}$ 


\subsection{Hydrogen}

The ultimate goal of this work was the development of a TALIF system to measure the atomic neutral density in hydrogen in a magnetic fusion device. Testing of the TALIF system in the relevant feedstock was therefore necessary to determine the performance and limitations of the system. Additionally, lower hybrid resonance effects, phenomena that have been previously shown to significantly improve plasma production in hydrogen, were investigated to determine their affect, if any, on neutrals.

\subsubsection{Parametric Scans in Hydrogen Plasma}

In monatomic helicon plasmas, significant decreases in neutral density have been observed with increasing rf power. ${ }^{9}$ This is consistent with increasing ionization leading directly to decreased neutral density. In a diatomic species, such as hydrogen, ionization and dissociation represent competing mechanisms for rf power absorption. As shown in Figure 6.13, both atomic neutral and plasma density increased with increasing power for fixed magnetic field and fill pressure in both hydrogen and deuterium. An $800 \%$ increase in rf power produced an increase in the dissociation fraction of $\mathrm{H}$ from $\sim 0.3$ to $\sim 1.3 \%$, and an increase in the ionization fraction, calculated with respect to the $\mathrm{H}_{2}$ fill density, from 0.005 to $0.02 \%$. The same increase in rf power in D produced an increase in the dissociation fraction from 0.5 to $2.5 \%$, and an increase in the ionization fraction from 0.045 to $0.1 \%$. This suggests that as power is increased both ionization and dissociation increased, leading to an increase in plasma density without the corresponding drop in neutral density observed in monatomic species.

The measured plasma density as a function of rf power is consistent with previous mea- 
surements in a similar size helicon source for low power, $<1 \mathrm{~kW}$, operation. ${ }^{10}$ In those experiments, higher density operation, $>10^{17-18} \mathrm{~m}^{-3}$ was not achieved until the rf power exceeded $1 \mathrm{~kW}$, and even then, proper magnetic field tuning, specifically operating near the lower hybrid resonance magnetic field, was necessary to achieve high densities.

In both hydrogen and deuterium, the atomic neutral density was $\sim 100 \mathrm{x}$ the plasma density, demonstrating that dissociation, not ionization, is the dominate power absorption mechanism in these weakly dissociated plasmas. The theoretical dissociation rate for $\mathrm{H}_{2}$ for $10 \mathrm{eV}$ electrons is $\sim 2.5 \mathrm{x}$ larger than the ionization rate of either atoms or molecules, ${ }^{11}$ thus the dissociation fraction is expected to be larger than the ionization fraction. However, the significant difference between the expected and measured ratio of ionization to dissociation implies other processes are controlling the final dissociation and ionization levels in the helicon source.

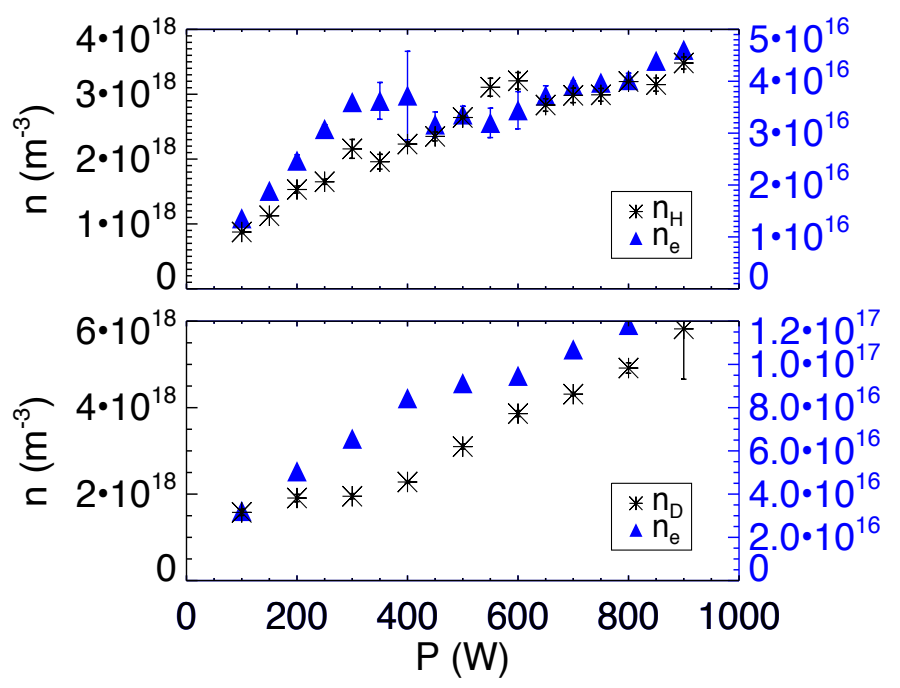

Figure 6.13: Neutral (black stars, left axis) and plasma (blue triangles, right axis) densities in H (top) and $\mathrm{D}$ (bottom) as a function of rf power. Constant discharge parameters were $630 \mathrm{G}$ magnetic field, $13 \mathrm{MHz}_{r f}, 8 \mathrm{mT}$ pressure. For all measured powers, both neutral and plasma densities were higher in deuterium than in hydrogen by a factor of 2 . 
Increasing rf power has also been shown to dramatically change the neutral density profile in noble gas helicons, with increases in power leading to hollow radial profiles. ${ }^{12}$ However, as shown in Figure 6.14, approximately uniform atomic neutral density profiles were found for all measured powers in hydrogen. The increase in the magnitude of the density in each profile is consistent with the increase with rf power shown in Figure 6.13.

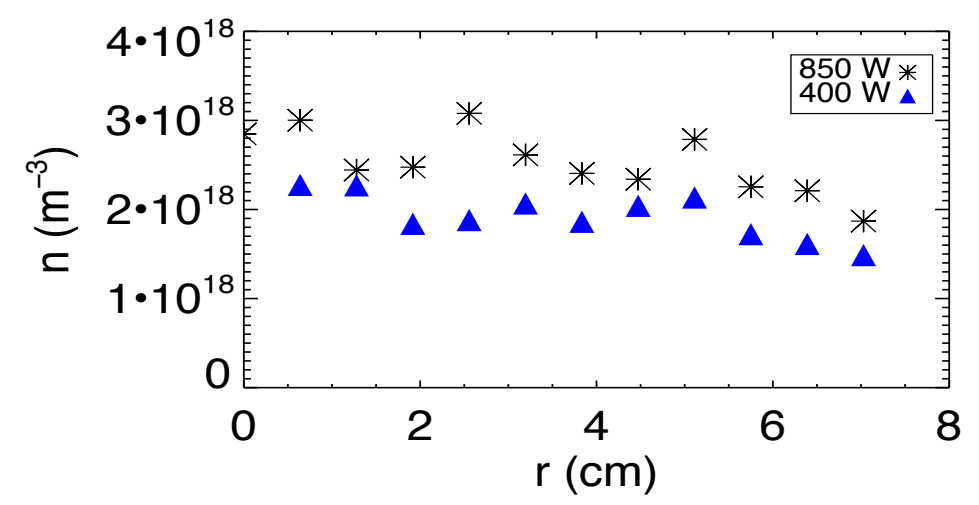

Figure 6.14: Neutral hydrogen density as a function of radius for two rf powers.

The hollow profiles observed in heavy ion helicons have been explained by neutral depletion, i.e., a decrease in neutral density beyond that due solely to ionization. ${ }^{8,9,13}$ As described in Chapter 1, two mechanisms have been proposed to explain neutral depletion. In the first, neutral particles enter the plasma and are ionized. The freshly created ions are then accelerated out of the plasma at the sound speed, $c_{s}=\sqrt{\frac{k_{B} T_{e}}{m_{i}}}$, by a sheath electric field. The ions are then neutralized in the edge region and may diffuse back into the plasma at the thermal speed, $v_{t h}=\sqrt{\frac{k_{B} T_{n}}{m_{n}}}$, where $T_{n} \ll T_{e}$. The large difference in the speeds results in a net decrease in the neutral density in the center of the plasma. The second mechanism is neutral expulsion from high plasma density regions by ion-neutral collisions to maintain pressure balance between the cold neutrals and hot plasma.

No matter what the exact mechanism for neutral depletion, the lack of neutral depletion 
in hydrogen (deuterium) plasmas results from the low levels of ionization. The neutral ionization length is

$$
\lambda_{n}=\frac{v_{n}}{n_{e}<\sigma v>_{i o n}},
$$

where $v_{n}$ is the neutral velocity, $n_{e}$ the electron density, and $\langle\sigma v\rangle_{i o n}$ is the electron energy dependent ionization rate. The penetration length is $\sim 10 \mathrm{~m}$ for the measured atomic neutral temperatures (see Figure 6.15) and plasma densities, and assuming an electron temperature of $10 \mathrm{eV}$. This is much larger than the chamber size. Therefore, the large lengths scales of atomic neutral diffusion, not ionization, yields a neutral density profile that is constant across the chamber diameter. It should be noted here that in all measured configurations

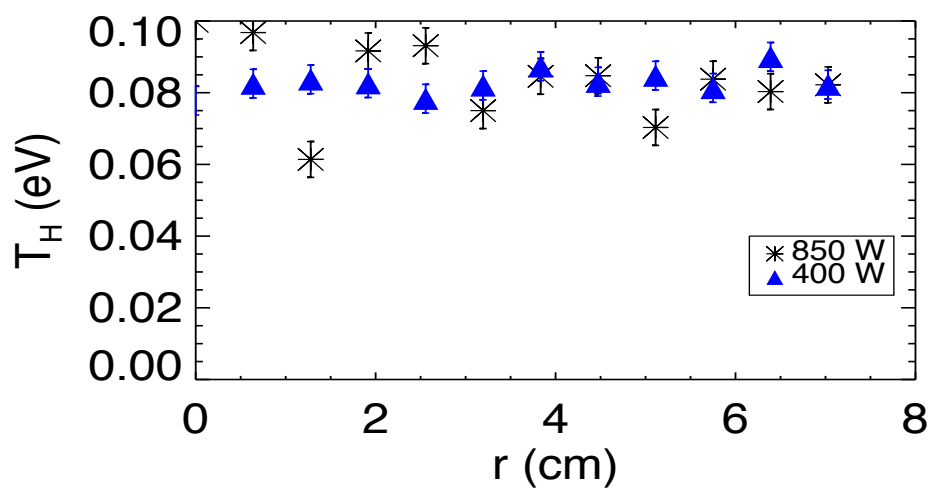

Figure 6.15: Neutral hydrogen temperature versus radius. Temperature is constant even as the density decreases in the edge. No changes in neutral temperature were measured with varying source parameters.

the neutral temperature was $\sim 0.08 \mathrm{eV}$, almost $3 \mathrm{x}$ room temperature. Thus, the neutral penetration length did not vary significantly between source parameters. Conventional wisdom is that the energy of collisionally dissociated neutrals is $2 \mathrm{eV} .{ }^{14}$ However, in these measurements of ground state hydrogen in low temperature plasma, the temperature is clearly much lower than $2 \mathrm{eV}$. One possibility is there is a hot neutral component. However, the measured hydrogen neutral velocity distribution function is well described by single Maxwellian distri- 
bution, rather than the sum of two distributions, see Figure 6.16. Further investigations of the evolution of the neutral temperature are described in Chapter 7.

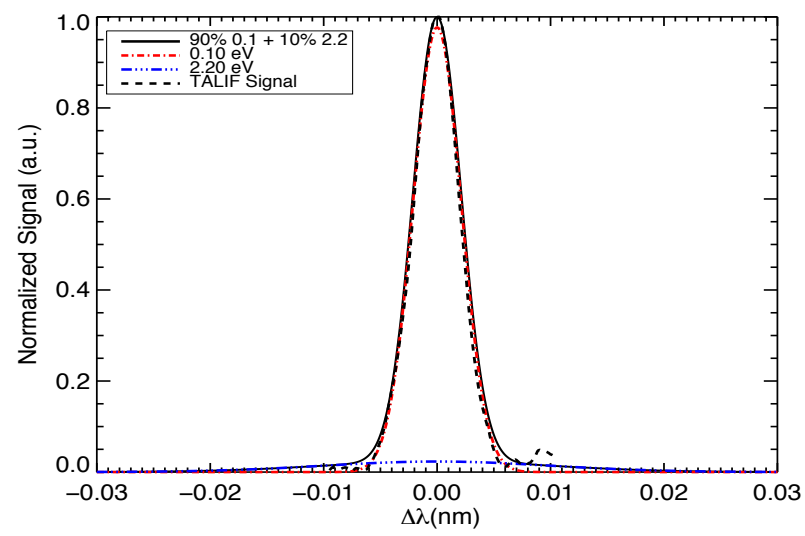

Figure 6.16: Distribution for two temperature populations. Dashed line are the experimental measurement.

Significant changes in both the plasma and neutral densities have also been observed in both monatomic and diatomic helicon sources when the driving frequency approaches the lower hybrid frequency. ${ }^{10,15}$ As noted previously, as the lower hybrid frequency approaches the driving frequency, significant damping of slow waves can occur leading to dramatic increases in plasma density. ${ }^{5}$

The lower hybrid frequency is

$$
\frac{1}{\omega_{l h}^{2}}=\frac{1}{\omega_{c i} \omega_{c e}}+\frac{1}{\omega_{p i}^{2}+\omega_{c i}^{2}},
$$

where $\omega_{c i}$ and $\omega_{c e}$ are the ion and electron cyclotron frequencies and $\omega_{p i}$ is the ion plasma frequency. In the high density, limit only the magnetic field determines the resonance fre- 
quency,

$$
\omega_{L H-H D} \cong \sqrt{\omega_{c i} \omega_{c e}}
$$

In the low density limit the lower hybrid frequency becomes

$$
\omega_{L H-L D} \cong \omega_{p i}
$$

For these experiments, the driving frequency was held fixed at $13 \mathrm{MHz}$. In the low density limit, a plasma density of $\sim 4 \times 10^{15} \mathrm{~m}^{-3}$ matches the lower hybrid resonance to the driving frequency. Except at the lowest fill pressures, all measured plasma densities in these experiment result in conditions well above the low density limit. Thus, the high density limit is a good approximation for determining the location of the lower hybrid resonance in these plasmas.

At a magnetic field strength of $\sim 200 \mathrm{G}(280 \mathrm{G})$ the atomic ion lower hybrid resonance matches the antenna frequency for hydrogen (deuterium). As shown in Figure 6.17, no increase in either atomic neutral or plasma production is observed at, or near, this resonance in either species. Additionally, no change in atomic neutral or plasma density was measured at the $\mathrm{H}_{2}{ }^{+}\left(\mathrm{D}_{2}{ }^{+}\right), 280 \mathrm{G}(400 \mathrm{G})$, or $\mathrm{H}_{3}{ }^{+}\left(\mathrm{D}_{3}{ }^{+}\right), 350 \mathrm{G}(485 \mathrm{G})$, resonances. This suggests that there is no coupling to the molecular ion resonances either. The lack of LH resonance effects is counter to previous moderate and high power hydrogen helicon experiments, in which significant, $\sim 3 \mathrm{x}$, increases in plasma density were measured near the LH resonance. ${ }^{10,15}$ In those experiments, the power density was $3 \mathrm{MW} / \mathrm{m}^{3}$ compared to $0.8 \mathrm{MW} / \mathrm{m}^{3}$ in these experiments. As a consequence of the higher power density, the plasma density was also larger in those experiments, $>10^{17} \mathrm{~m}^{-3}$. This suggests that a sufficient plasma density is necessary for LH resonance effects to occur. It has also been shown that for sufficiently high 
power, high density can be achieved away from the resonance, ${ }^{16}$ though the power density was again much larger, $\sim 15 \mathrm{MW} / \mathrm{m}^{3}$.

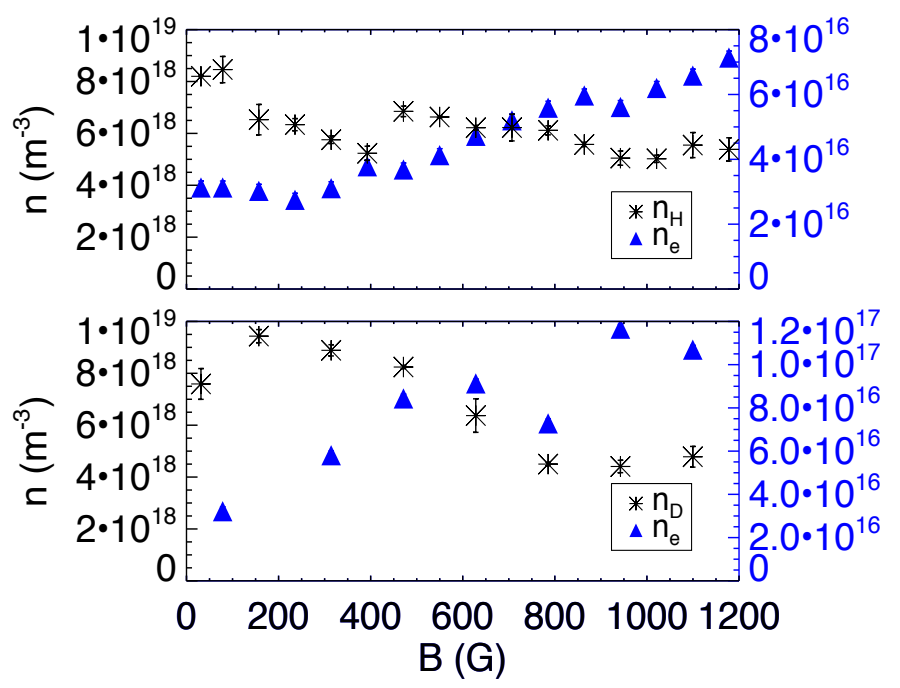

Figure 6.17: Neutral hydrogen (black stars, left axis) and plasma (blue triangles, right axis) densities in $\mathrm{H}$ (top) and D (bottom) as a function of magnetic field. The lower hybrid resonance matched the driving frequency at $200 \mathrm{G}$. Constant discharge parameters were $13 \mathrm{MHz} \mathrm{rf}, 600 \mathrm{~W}$ power, $8 \mathrm{mT}$ pressure.

As was observed with increasing rf power, the atomic neutral density profile was approximately constant with increasing magnetic field (Figure 6.19). Plasma density (Figure 6.19) displayed a considerable amount of change with increasing field. At low field strength the profile was relatively flat, with no change in the magnitude of the density with increasing field. At higher field strengths the profile became centrally peaked, with the amplitude on axis increasing with increasing field strength. At these higher field strengths the profile is well described by $1-(r / a)^{2}$, where $a$ is the tube radius.

A change in the plasma density profile is expected when switching from the inductive to helicon operating modes due to a change in the location of plasma creation. As was described in Chapter 2, in the inductively coupled mode the plasma is sustained and heated 
by the induction of currents. The energy penetration is limited to the skin depth, resulting in power deposition near the edge of the plasma and a flat plasma density profile. In helicon mode the plasma is sustained and heated via resonant absorption of an rf wave. The wave can penetrate into the interior of the plasma, resulting in power deposition in the center of the plasma, and a centrally peaked plasma density profile. ${ }^{17}$ Helicon mode is characterized by high plasma density, and satisfies the dispersion relation

$$
n=\frac{k B_{0}}{\mu_{0} e}\left(\frac{k_{\|}}{\omega}\right),
$$

where $n$ is the density, $k_{\|}$is the parallel wavenumber, $k$ is the magnitude of the wavevector $\left(k=\sqrt{k_{\perp}^{2}+k_{\|}^{2}}\right), B_{0}$ is the background magnetic field, $\mu_{0}$ is the permeability of free space, $e$ is the elementary charge, and $\omega$ is the wave frequency. ${ }^{18}$ As shown in Figure 6.18, for the given magnetic fields in these experiments, $100 \mathrm{~s} \mathrm{G}$, and assuming $k_{\|}$to be the inverse tube length and $k_{\perp}$ to be the inverse tube radius, the dispersion relation is not fulfilled like it was for krypton (even adjusting the density by a factor of 10.) Thus the source is not operating in the helicon mode even though a transition to a centrally peaked profile is observed.

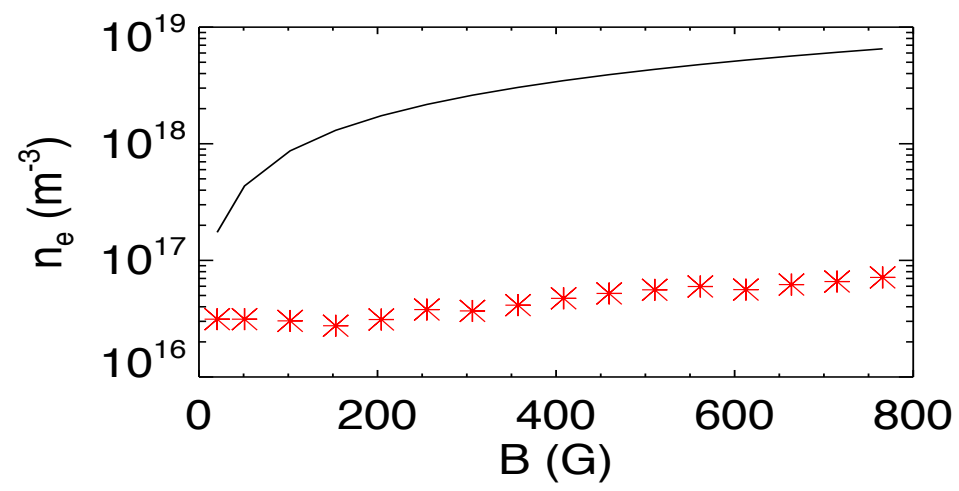

Figure 6.18: Helicon dispersion relation (solid line) and measured plasma density (red stars) in hydrogen. 

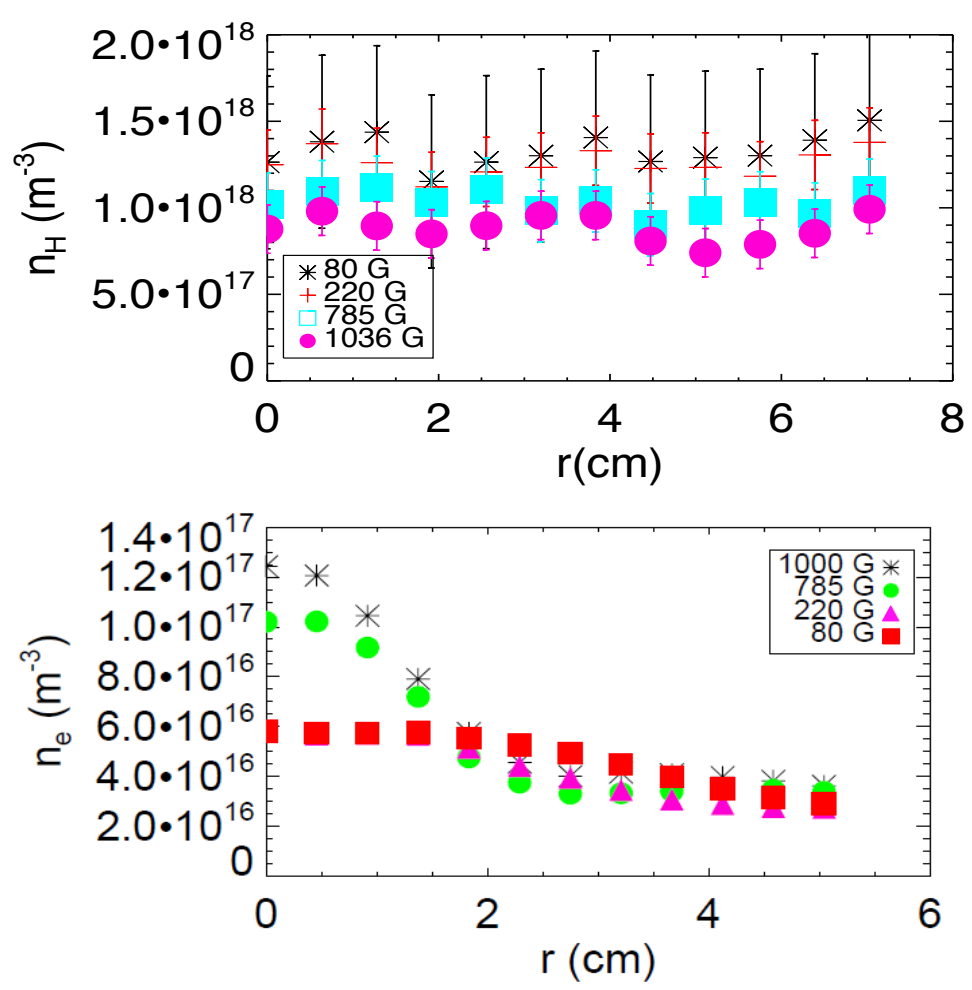

Figure 6.19: Neutral hydrogen (top) and plasma (bottom) densities as a function of radius above and below the lower hybrid resonance.

It is interesting to note that while it is relatively easy to visually recognize helicon mode in noble gas species, it is difficult to do the same in hydrogen. Helicon mode is characterized by a bright, high density core, with significant ion emission. In a high density, highly ionized hydrogen plasma, the ions are fully stripped, having no emission lines. Thus, the core in hydrogen will be invisible, making visual identification of helicon mode very difficult, if not impossible. Even if the source was operating in helicon mode for high magnetic field strengths, the center peaked plasma density profile would be the only way to recognize it.

Increasing fill pressure produced a nearly linear increase in neutral density in both $\mathrm{H}$ and $\mathrm{D}$, indicating a nearly constant dissociation fraction of $\sim 0.4 \%$ in $\mathrm{H}$ and $\sim 2 \%$ in $\mathrm{D}$, as seen in Figure 6.20. In $\mathrm{H}$, the plasma density was low at low pressure, $\sim 10^{15} \mathrm{~m}^{-3}$ below 6 
mTorr. Above 6 mTorr, the plasma density jumped by nearly an order of magnitude and remained roughly constant. The constant plasma density above 6 mTorr in $\mathrm{H}$ corresponds to a decreasing ionization fraction, from $\sim 0.02 \%$ at 6 mTorr to $\sim 0.005 \%$ at $20 \mathrm{mTorr}$. The

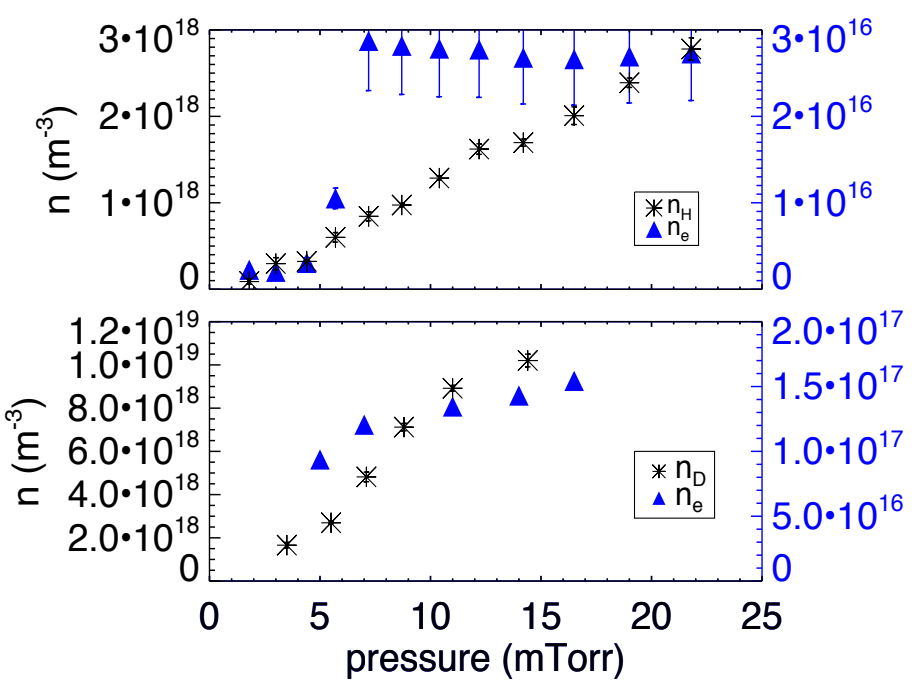

Figure 6.20: Neutral (black stars, left axis) and plasma (blue triangles, right axis) densities in $\mathrm{H}$ (top) and D (bottom) as a function of fill pressure as a function of equilibrium fill pressure. The jump in $\mathrm{H}$ plasma density at $6 \mathrm{mT}$ indicates a switch from capacitive coupling to inductive. Constant discharge parameters were $630 \mathrm{G}$ field, $13 \mathrm{MHz}$ rf, and $600 \mathrm{~W}$ power.

jump in density suggests an operating mode change. The operating mode is governed by the electron skin depth. When the skin depth is larger than the system size the source is in a capacitive mode. As the electron density increases the skin depth decreases. When the skin depth is less than the system size the source is operated in either inductive or helicon mode. As shown in Figure 6.21, at a plasma density of $\sim 4.5 \times 10^{16} \mathrm{~m}^{-3}$ the electron skin depth is less than the tube radius, $2.5 \mathrm{~cm}$. At low pressure, the plasma density is an order of magnitude lower than the capacitive-to-inductive density limit, $\sim 4 \times 10^{15} \mathrm{~m}^{-3}$. This suggests that the jump in density cannot be explained by a mode change. However, as with krypton, 
the measurements presented here were made in the expansion region. If plasma is created under the antenna in the source region, then diffuses into the expansion region, the plasma density is larger in the source than expansion regions. Thus, while the plasma density in the expansion region is inconsistent with a mode change, if the plasma density in the source were $10 \mathrm{x}$ larger, the density limit for a capacitive-to-inductive change is satisfied, and the density jump can be explained by a transition from a capacitive to an inductive mode.

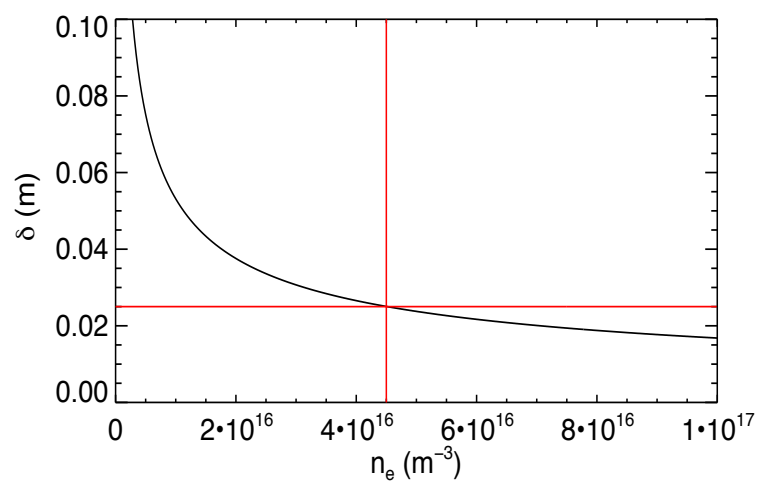

Figure 6.21: Electron skin depth as function of electron density. Horizontal line is the radius of the Pyrex tube.

It has been shown in atomic species that increasing fill pressure can also increase plasma density, due to an increase in fuel. The fact that the measured atomic neutral densities in $\mathrm{H}$ and D increase linearly with fill pressure indicates that dissociation is the dominant power absorption mechanism. The atomic neutral density production rate scales as

$$
\frac{d n_{H}}{d t} \sim n_{e} n_{H_{2}}<\sigma v>_{d i s s}
$$

where $n_{e}$ and $n_{H_{2}}$ are the plasma and molecular densities, and $\langle\sigma v\rangle_{\text {diss }}$ is the electron energy dependent dissociation rate. Only for constant plasma density and temperature will the atomic neutral density scale linearly with the molecular fill density. If ionization were an 
equal power absorption mechanism to dissociation, the plasma density would increase with increasing fill pressure, and the atomic neutral density will increase faster than linearly.

The plasma density in D showed no such mode change, and even at the lowest measured fill pressure, 5 mTorr, the plasma density, $1 \times 10^{17} \mathrm{~m}^{-3}$, was above the $4.5 \times 10^{16} \mathrm{~m}^{-3}$ limit for capacitive mode operation. Thus, the source was always operated in the inductive coupling mode in D. As was observed in the other parametric scans, the D atomic neutral and plasma densities were larger than the $\mathrm{H}$ atomic neutral and plasma densities at all measured fill pressures.

The 2-3x larger atomic neutral and plasma densities measured in deuterium plasmas are unexpected as deuterium and hydrogen have similar dissociation and ionization potentials. Other groups have measured differences in the plasma density between the two species in helicon sources. ${ }^{10}$ However, they measured higher plasma densities in H, rather than D. There is no clear reason for why there should be a difference between the two species, however it has been argued that in low density helicons, slow waves, whose dispersion relation is ion mass dependent, can strongly affect coupling. ${ }^{19}$

One possibility for the difference in the density behavior in $\mathrm{H}$ and $\mathrm{D}$ is the difference in transport between the two species. D, having a higher mass, and same temperature as H, $<0.1 \mathrm{eV}$, is less mobile. Thus, D neutrals (molecular and atomic) and ions take longer to diffuse out of the chamber than $\mathrm{H}$ neutrals and ions. The increase in diffusion time allows for more dissociating and ionizing collisions, increasing both the atomic neutral and plasma densities.

The most likely reason for the inability to achieve high plasma density operation in either hydrogen or deuterium for any set of source parameters is power absorption through molecular dissociation. In all measurements presented here the dissociation fraction was 
between 10 and 100 times the ionization fraction, 0.1-1\% dissociation fraction compared to $0.01-0.1 \%$ ionization fraction. This indicates a significant amount of power (energy) is going into creating atomic neutrals rather than plasma. Plasma densities of $10^{17}-10^{18} \mathrm{~m}^{-3}$ are routinely achieved in monatomic species ( $\mathrm{Ar}, \mathrm{He}, \mathrm{Kr}$ ) in $\mathrm{CHEWIE}$ for conditions that only produce $10^{16} \mathrm{~m}^{-3}$ in hydrogen and deuterium. The differences in noble and molecular species plasma densities observed in CHEWIE are consistent with previous measurements in a similar helicon source, ${ }^{10}$ suggesting that in general molecular species are non-ideal helicon fuel gases.

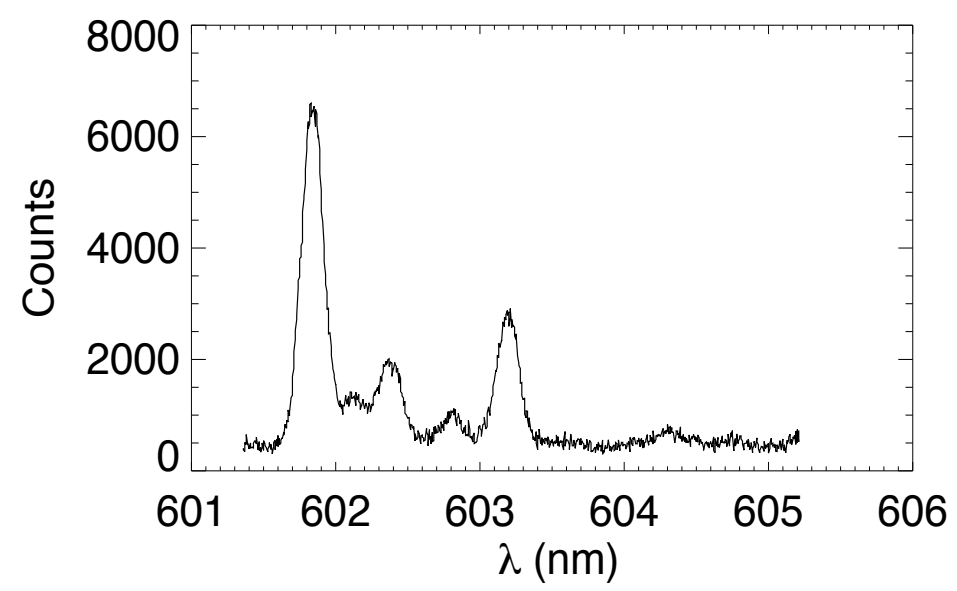

Figure 6.22: $\mathrm{H}_{2}$ emission spectrum showing excitation of Q-branch of Fulcher- $\alpha$ band.

In addition to power absorption through molecular dissociation, it is possible that a significant fraction of the power is absorbed into neutral excitation. The excitation rate for the plasma temperatures in CHEWIE is $\sim 3$ orders of magnitude larger than the ionization rate of molecules or atoms and the dissociation rate, ${ }^{20}$ thus it is assumed that the majority of rf power is absorbed via neutral or molecular excitation. Emission spectra reveal both excited molecular (shown in Figure 6.22), and atomic lines (shown in Figure 6.23). This could also explain the vast difference in the measured neutral and plasma densities. Molecules in excited 


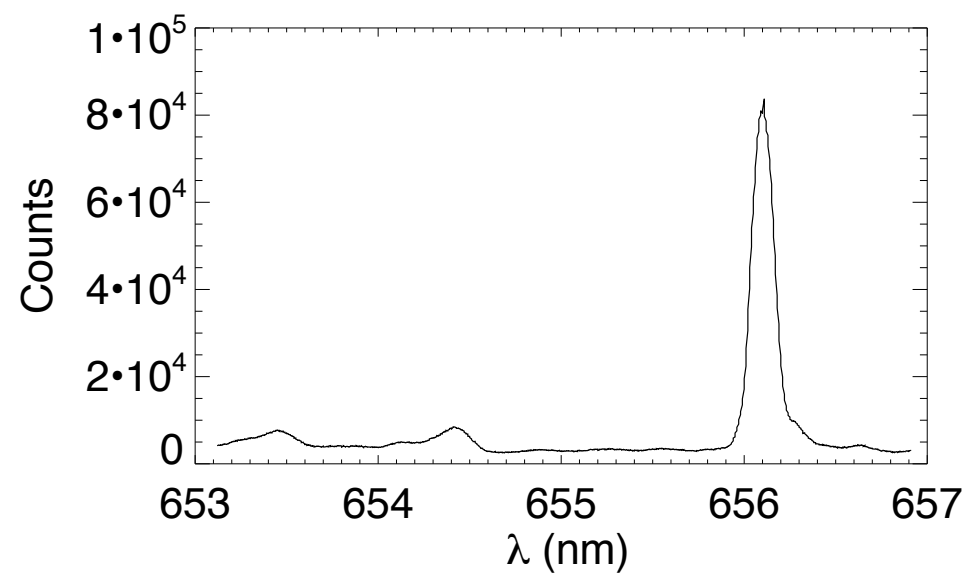

Figure 6.23: $\mathrm{H}$ emission spectrum showing excitation of $H_{\alpha}$ transition.

vibrational states are preferentially dissociated, thus increasing only the neutral density.

\subsubsection{Axial Profiles in Hydrogen Plasma}

Previous studies in molecular helicon sources reported a slight axial gradient in the atomic neutral density, with the largest density of neutrals in the antenna region. ${ }^{21}$ Similar behavior is observed in hydrogen in CHEWIE, though the axial density gradient is much larger than those previously observed. As was observed in the radial neutral density profiles, axial neutral density profiles displayed the same shape for each measured power, with the baseline increasing with increasing power, see Figure 6.24. For a uniform background of molecular gas, it is expected that the atomic neutral density is highest where the electron density is largest, assuming uniform electron temperature. This is because atomic neutrals are the product of dissociating collisions between molecules and free electrons. The data shown in Figure 6.24 suggest if the above expectation is true, the electron density is highest in the antenna region, as is expected. It is surprising, given the uniform radial atomic density profiles in the expansion region, that the axial profile displays such a dramatic gradient. 


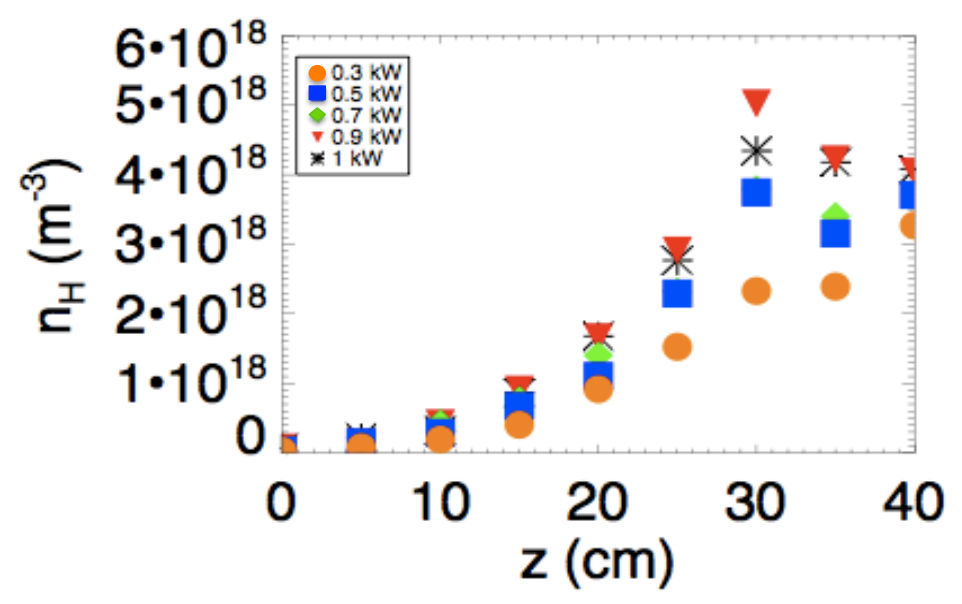

Figure 6.24: Axial atomic hydrogen density for five rf powers. $z=40$ corresponds to the bottom of the antenna. $z=0$ is the top of the glass tube.

Even for high power, high field operation, when the radial plasma density profile is centrally peaked, the neutral density profile in the expansion region remains uniform. Along the axis of the source, the large plasma densities under the antenna are a source of dissociation. No such source exists in the expansion region. Thus, the sharp increase in neutral density under the antenna simply reflects the location of the source of neutral production.

\subsection{Mixed Species}

In pure $\mathrm{H}$ plasma, the atomic neutral density is relatively low, $\sim 1 \%$ of the fill density. In an attempt to increase the atomic $\mathrm{H}$ density, $\mathrm{Kr}$ was added to the plasma. As shown in the Section 6.1.1, Kr is very efficient plasma creator, with plasma densities $>10^{18} \mathrm{~m}^{-3}$. The $\mathrm{Kr}$ in an $\mathrm{H}$ plasma would act as an electron donor, increasing the electron density in the plasma, with the added electrons increasing the $\mathrm{H}$ density through dissociating collisions. Additionally, the $\mathrm{Kr}$ ionization potential, $\sim 14 \mathrm{eV}$, is close to both the $\mathrm{H}, 13.6 \mathrm{eV}$ and $\mathrm{H}_{2}$, 15.4 eV, ionization potentials. Thus, no species would be preferentially ionized. Previous 
studies in mixed species helicon plasmas have shown that if the species have very disparate ionization potentials, the one with the lower potential will be preferentially ionized over the other. ${ }^{22}$ This can starve the plasma of electrons as the fraction of species with higher ionization potential becomes dominant.

For constant $\mathrm{H}_{2}$ fill density, adding $\mathrm{Kr}$ increased the $\mathrm{H}$ density by nearly a factor of 2 , see Figure 6.25. Due to experimental constraints, pressure measurements were not available during these measurements, therefore the total fill pressure was not known and a comparison cannot be made with a pure $\mathrm{H}$ plasma. However, the $\mathrm{H}$ atomic density is on the same scale as was produced in a pure $\mathrm{H}$ plasma for similar rf power and magnetic field strength. Thus, the addition of $\mathrm{Kr}$ did not significantly increase the $\mathrm{H}$ atomic density. Langmuir probe data are needed to determine if the addition of $\mathrm{Kr}$ increased the plasma density. However, due to the addition of a second ionic species, analysis of such data is problematic, as knowledge of the ionic fraction of the plasma is to interpret the I-V characteristic.

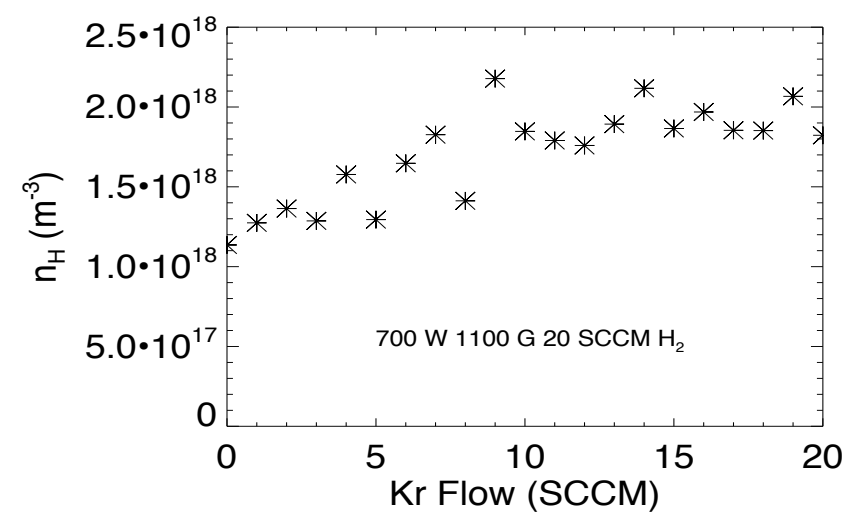

Figure 6.25: Atomic hydrogen density in mixed H-Kr plasma. Constant $20 \mathrm{SCCM} \mathrm{H} \mathrm{H}_{2}$ flow rate. 


\section{References}

[1] R. W. Boswell, "Very efficient plasma generation by whistler waves near the lower hybrid frequency," Plasma Physics and Controlled Fusion, vol. 26, pp. 1147-1162, 1984.

[2] P. A. Keiter, E. E. Scime, and M. M. Balkey, "Frequency dependent effects in helicon plasmas," Physics of Plasmas, vol. 4, pp. 2741-2747, 1997.

[3] S. Houshmandyar and E. E. Scime, "Enhanced neutral depletion in a static helium helicon discharge," Plasma Sources Science and Technology, vol. 21, p. 035008, 2012.

[4] S. Cho, "The role of the lower hybrid resonance in helicon plasmas," Physics of Plasmas, vol. 7, pp. 417-423, 2000.

[5] J. L. Kline, E. E. Scime, R. F. Boivin, A. M. Keesee, and X. Sun, "Slow wave ion heating in the helix helicon source," Plasma Sources Science and Technology, vol. 11, pp. 413-425, 2002.

[6] D. G. Miljak and F. F. Chen, "Density limit in helicon discharges," Plasma Sources Science and Technology, vol. 7, pp. 537-549, 1998.

[7] P. A. Keiter, Experimental Investigation of Ion Temperature Anisotropy Driven Instabilities in a High Beta Plasma. PhD thesis, West Virginia University, 1999.

[8] R. M. Magee, M. E. Galante, J. C. Jr., G. Lusk, D. W. McCarren, and E. E. Scime, "Neutral depletion and the helicon density limit," Submitted to Physics of Plasmas, 2013.

[9] J. Gilland, R. Breun, and N. Hershkowitz, "Neutral pumping in a helicon discharge," Plasma Sources Science and Technology, vol. 7, pp. 416-422, 1998. 
[10] Y. Sakawa, T. Takino, and T. Shoji, "Contribution of slow waves on production of high-density plasmas by $\mathrm{m}=0$ helicon waves," Physics of Plasmas, vol. 6, pp. 4759$4766,1999$.

[11] K. Sawada and T. Fujimoto, "Effective ionization and dissociation rate coefficients of molecular hydrogen in plasma," Journal of Applied Physics, vol. 78, pp. 2913-2924, 1995.

[12] G. R. Tynan, "Neutral depletion and transport meachanisms in large-area high density plasma sources," Journal of Applied Physics, vol. 86, p. 5356, 1999.

[13] L. Liard, A. Aanesland, and P. Chabert, "Dynamics of neutral gas depletion investigated by time- and space- resolved measurements of xenon atom ground state density," Journal of Physics D: Applied Physics, vol. 45, pp. 235201-235206, 2012.

[14] M. A. Lieberman and A. J. Lichtenberg, Principles of Plasma Discharges and Materials Processing. Wiley \& Sons, 2005.

[15] H. Kikuchi, Y. Fukui, Y. Sakawa, and T. Shoji, "Damping of waves in helicon-wave range of frequency in molecular- and rare-gas plasmas," Physics of Plasmas, vol. 12, pp. 521-526, 2003.

[16] Y. Mori, H. Nakashima, F. W. Baity, R. H. Goulding, M. D. Carter, and D. O. Sparks, "High density hydrogen helicon plasma in a non-uniform magnetic field," Plasma Sources Science and Technology, vol. 13, pp. 424-435, 2004.

[17] A. R. Ellingboe and R. W. Boswell, "Capacitive, inductive and helicon-wave modes of operation of a helicon plasma source," Physics of Plasmas, vol. 3, pp. 2797-2804, 1996. 
[18] F. F. Chen, "Plasma ionization by helicon waves," Plasma Physics and Controlled Fusion, vol. 33, pp. 339-364, 1991.

[19] M. D. Carter, F. W. Baity, R. H. Goulding, E. F. Jaeger, and J. P. S. F. R. ChangDiaz, and, "Light ion helicon plasma sources," AIP Conference Proceedings, vol. 595, pp. 465-472, 2001.

[20] R. K. Janev, W. D. Langer, K. Evans, and D. E. Post, Elementary Processes in Hydrogen-Helium Plasmas. Springer-Verlag, 1987.

[21] G. R. Tynan, A. D. Bailey, G. A. Campbel, R. Charatan, A. de Chambrier, G. Gibson, D. J. Hemker, K. Jones, A. Kuthi, T. S. C. Lee, and M. Wilcoxson, "Characterization of an azimuthally symmetric helicon wave high density plasma source," Journal of Vacuum Science \& Technology, A: Vacuum, Surfaces, and Films, vol. 15, pp. 2885-2892, 1997.

[22] I. Biloiu, Laser Induced Fluorescence studies of ion acceleration in single and multiple species in expanding helicon plasma. PhD thesis, West Virginia University, 2009. 


\section{Chapter 7}

\section{Time Resolved TALIF Measurements}

The data presented in the previous chapter were obtained with the source operating in steady-state. As a result of the short duration of the laser pulse in the TALIF system, time resolved measurements were also possible, with little alteration to the experimental setup. Time resolved neutral density and neutral temperature measurements reveal the timescale of neutral depletion in krypton and neutral growth through dissociation in hydrogen. Addition-

ally, these measurements provide an explanation for the low neutral temperature observed in steady-state hydrogen plasma.

\section{$7.1 \quad$ Krypton}

In high density plasma, neutral depletion places an upper limit on the achievable plasma density. The lack of neutrals effectively starves the plasma of fuel, preventing further increase in plasma density. Neutral depletion is a transient phenomenon. By making very fast, time-resolved TALIF measurements, the time scale of neutral decay in the source region is measured. Neutral density measurements in the expansion region demonstrate neutral loss 
in the plasma core, and neutral growth in the edge region, consistent with neutral expulsion.

The rate of change of krypton neutrals is

$$
\frac{d n_{n}}{d t}=S+n_{i} n_{e}<\sigma v>_{r e c}-n_{n} n_{e}<\sigma v>_{i o n}-P
$$

where $S$ is the source term, $n_{n}, n_{i}$, and $n_{e}$ are the neutral, ion, and electron density, respectively, $\langle\sigma v\rangle_{r e c}$ is the radiative recombination rate, $\langle\sigma v\rangle_{i o n}$ is the electron impact ionization rate, and $P$ is a sink term. Under the assumption that on a short timescale, neutrals are lost primarily to ionization, the above equation can be solved as

$$
n_{n}(t)=\frac{n_{n, i}}{1-A \exp \left(n_{t o t}<\sigma v>_{\text {ion }} t\right)}
$$

where $n_{t o t}$ is the total number of particles, $n_{t o t}=n_{n}+n_{e}, n_{n, i}$ is the initial number of neutrals, and $A=\left(1-n_{t o t} / n_{n, i}\right)$. A fit of this solution is shown in Figure 7.1, with the ionization rate and initial electron density as free parameters. The ionization rate implied by the fit is for an electron temperature $T_{e}=37 \mathrm{eV} .{ }^{2}$ This is much larger than the $5 \mathrm{eV}$ temperatures normally measured in helicon sources. However, some have proposed that early in the discharge there is a population of energetic electrons that are responsible for the initial plasma creation. ${ }^{1}$ High time resolution electron temperature measurements in the antenna region are needed to determine if these energetic electrons exist early in the discharge. 


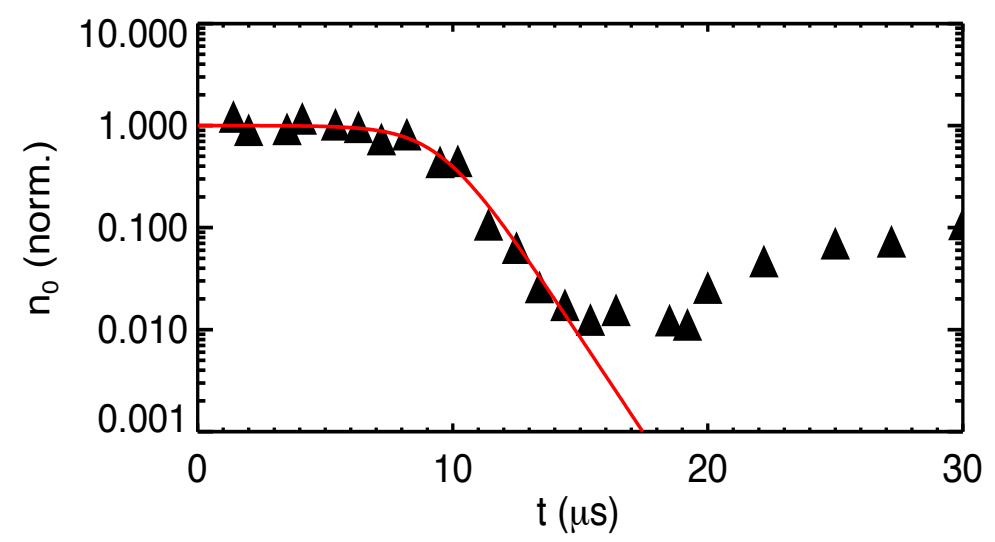

Figure 7.1: Normalized time resolved neutral $\mathrm{Kr}$ density in the source region for $4 \mathrm{~kW}$ input power. Fit is solution to coupled differential equation.

While the decrease in neutral density in the source region occurs within $15 \mu \mathrm{s}$, the decrease in the expansion region is much slower, $\sim 15 \mathrm{~ms}$, see Figure 7.3. As the neutral density in the core drops the neutral density in the edge rises on the same timescale. This is consistent with ion-neutral collisions in the core expelling neutrals from the high plasma density region into the low plasma density edge.

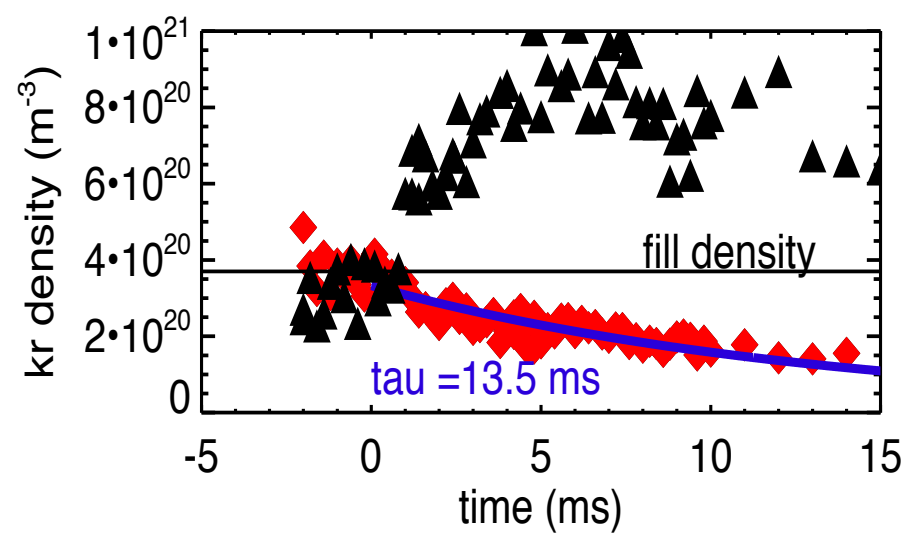

Figure 7.2: Time resolved neutral $\mathrm{Kr}$ density for two radial locations, $r=0$ (stars) and $r=6.9 \mathrm{~cm}$ (diamonds) in the expansion region. Vertical lines represent start $(t=0)$ and end $(t=25 \mathrm{~ms})$ of plasma pulse. $^{2}$ 
The difference in the timescale of neutral decay between the source and expansion regions can be explained by diffusion. Plasma is initially created very rapidly, $\sim 10 \mu$ s, by energetic electrons in the antenna region. This plasma then diffuses out of the antenna region and into the expansion region. As the plasma expands it cools and no longer efficiently ionizes the cold neutral gas in the expansion region. Thus in the expansion region, ionization does not dominate the neutral dynamics. However, the neutrals respond to the increase in plasma pressure. Ion-neutral collisions force neutrals into the edge, thus the decrease in core neutral density and increase in edge neutral density shown in Figure $7.3 .^{2}$

One of the consequences of increasing rf power is the $t=0$ density does not approach the fill density, see Figure 7.3. This suggests incomplete recombination, i.e., the recombination time is longer than the time between pulses. This can be seen more clearly when varying the pulse length at fixed frequency, see Figure 7.4. As the pulse length increases the $t=0$ neutral density decreases, evidence that the recombination time is longer than the plasma off time.

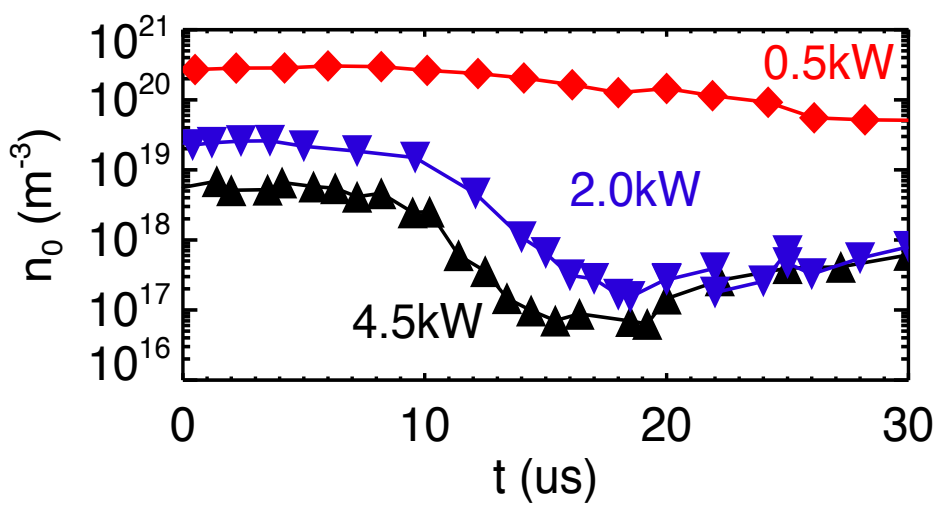

Figure 7.3: Time resolved neutral Kr density for three powers in the source region. 


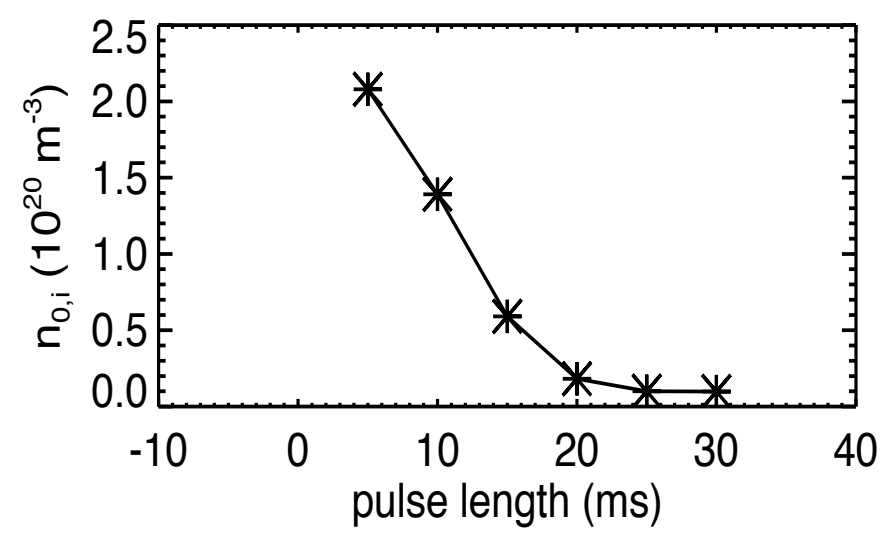

Figure 7.4: Neutral density as a function of pulse length for fixed frequency, $20 \mathrm{~Hz}$, in Kr. As the plasma pulse length increases, and the plasma off time decreases, the neutral density decreases. This suggests that the plasma is not completely recombining after the discharge, leading to a drop in the "plasma off" neutral density. ${ }^{2}$

\subsection{Hydrogen}

Atomic neutrals are a product in diatomic plasmas. Thus, they will have some finite growth time after the discharge is initiated. Time-resolved TALIF measurements made in the source region reveal that atomic neutral hydrogen increases on the sub-ms timescale, but does not start until $50 \mu$ s after the discharge is initiated, counter to the results in Kr, in which the neutral decay in the source began on the sub- $\mu$ s timescale.

Similar behavior is seen in the expansion chamber. The neutral density grows very rapidly, in the first $2 \mathrm{~ms}$, then levels off, see Figure 7.6. The neutral density in the expansion chamber is a factor of 10 lower than the density measured in the same location in a $\mathrm{CW}$ plasma. The difference between the pulsed and CW density points to some long time scale effect increasing the neutral density. As was discussed in $\mathrm{Kr}$, the recombination time scale is similar to the pulse time scale, $10 \mathrm{~s}$ ms. For long pulses, complete recombination does not 


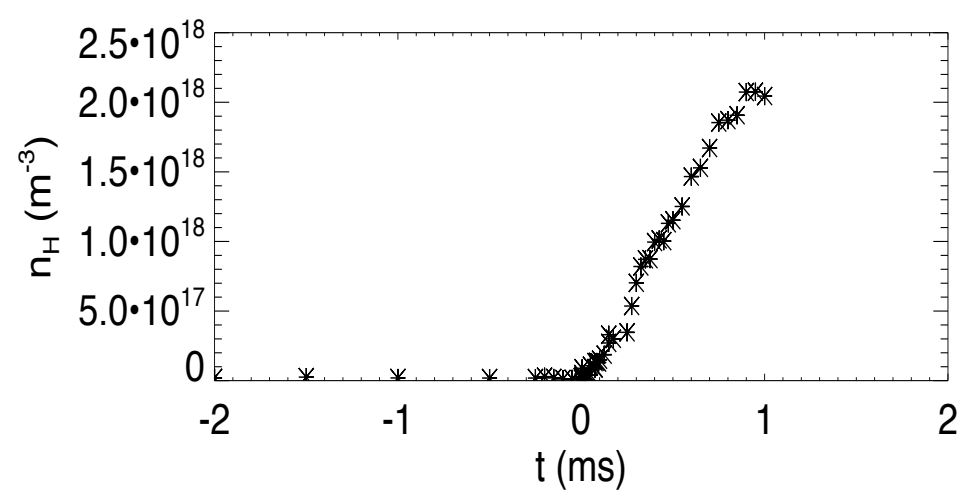

Figure 7.5: Time resolved neutral $\mathrm{H}$ density underneath the antenna. $0 \mathrm{~ms}$ corresponds to the start of the discharge.

occur. In $\mathrm{Kr}$, this resulted in a decrease in the plasma off neutral density with increasing pulses. In $\mathrm{H}$, if a portion of the neutral hydrogen is the result of proton-electron recombination, it will be affected by the pulse length, leading to lower measured neutral densities when pulsing. Varying duty cycle experiments, similar to those done in $\mathrm{Kr}$, are needed to confirm if incomplete recombination is leading to a decrease in neutral density.

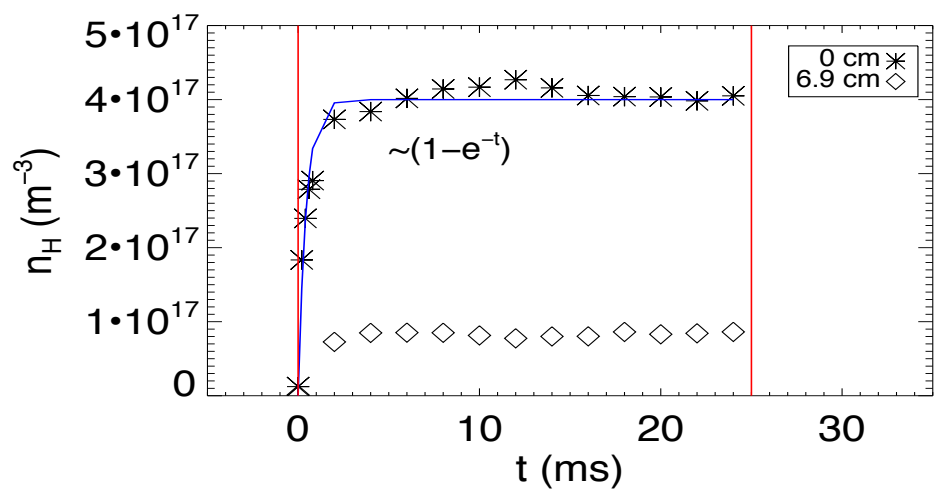

Figure 7.6: Time resolved neutral $\mathrm{H}$ density for two radial locations, $r=0$ (stars) and $r=6.9 \mathrm{~cm}$ (diamonds). Vertical lines represent start $(t=0)$ and end $(t=25 \mathrm{~ms})$ of plasma pulse.

The neutral density under the antenna is $\sim 10 \times$ the density measured in the expansion chamber, see Figures 7.5 and 7.6. The larger neutral density under that antenna makes sense if atomic neutrals are preferentially created in the antenna region via collisions with 
electrons accelerated by the rf wave. To determine if, as seen in krypton, neutrals are created in the source, then diffuse into the expansion region, sub $100 \mu$ s time-resolved neutral density measurements in the expansion region are needed because the time scale for diffusion of a $0.1 \mathrm{eV}$ hydrogen neutral atom is $\sim 100 \mu \mathrm{s}$. Unfortunately, the data shown in Figure 7.6 are limited to $100 \mu$ s time resolution, thus it cannot be determined whether the neutrals in the expansion region are the result of diffusion or are created locally.

In addition to measuring the neutral density as a function of time, time-resolved TALIF measurements also provided a measure of the neutral temperature as a function of time. This was used to determine if neutrals were "born" with high energies, then rapidly thermalized down to the temperature measured in steady-state. Measurements in the source region show some higher energy neutrals early in time (Figure 7.7), but these temperatures are still much less than the expected $2 \mathrm{eV}$. In the expansion region no neutral temperatures greater than $0.08 \mathrm{eV}$ were measured (the same temperature measured in steady-state) see Figure 7.8. These measurements demonstrate that neutrals are created with very low energies. From Tawara et al. "From the lowest excited states of $\mathrm{H}_{2}$, two kinds of $\mathrm{H}(1 s)$ atoms with different energies are produced: one is the near-zero energy atoms from the attractive $1 s \sigma^{2} \Sigma_{g}^{+}$state, resulting in dissociation into $\mathrm{H}^{+}$and $\mathrm{H}(1 s)$ and the other relatively high-energy atom $(\cong$ $7 \mathrm{eV}$ ) from the corresponding repulsive $2 p \sigma_{u}{ }^{2} \Sigma_{u}^{+}$state. In between there are a series of different channels contributing to the production of $\mathrm{H}(1 s)$ through $\mathrm{H}(1 s)+\mathrm{H}^{*}(n l)$ excitation. Only little is known of the behavior of $\mathrm{H}(1 s)$ atoms produced through dissociation of $\mathrm{H}_{2}$ molecules. In fact, because of the difficulties in detecting atoms in the ground state, their energy distributions and angular distributions have not yet been investigated in detail..." 3 The results shown in Figures 7.5 and 7.8 suggest that neutrals in CHEWIE are produced from a multi-step dissociation process, also known as pre-dissociation, in which electron 
impact first produces an excited molecule, then an ionizing dissociation.

$$
H_{2} \rightarrow H_{2}^{*} \rightarrow H^{+}+H(1 s)
$$

The resultant neutral atom is born at nearly zero energy.

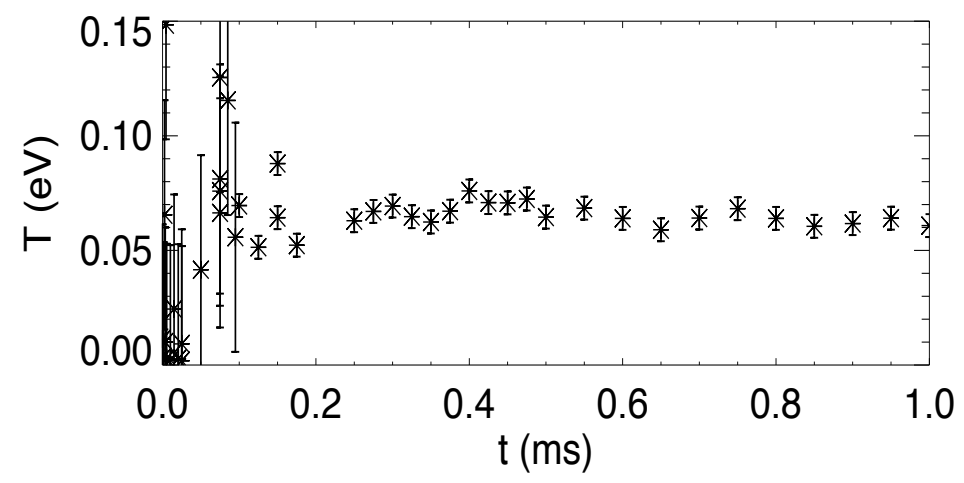

Figure 7.7: Time resolved neutral $\mathrm{H}$ temperature in the source region. Early in time, $<0.2 \mathrm{~ms}$, the neutral temperature is $2 \mathrm{x}$ higher than in steady-state, but it is still much lower than the expected 2 $\mathrm{eV}$.

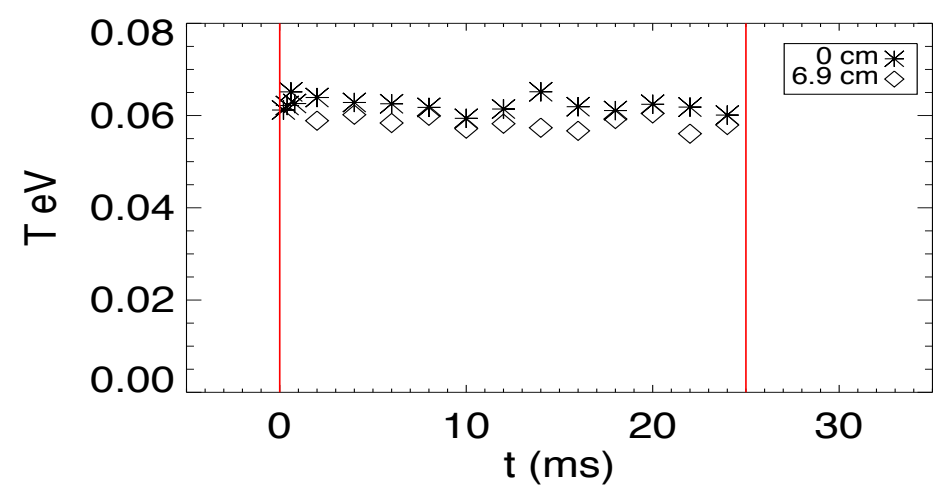

Figure 7.8: Time resolved neutral $\mathrm{H}$ temperature for two radial locations, $r=0$ (stars) and $r=6.9 \mathrm{~cm}$ (diamonds). Vertical lines represent start $(t=0)$ and end $(t=25 \mathrm{~ms})$ of plasma pulse. Neutral temperature is $\sim 0.07 \mathrm{eV}$ at all measured times. This is the same temperature as is found in steady-state.

If pre-dissocation is the dominant neutral production process in the experiment it raises the question of why the neutral density is much larger, $\sim 100 \mathrm{x}$, than the plasma density, as 
an one electron and one proton is produced for every one atom. It is possible that early in time, the plasma and atom densities are equal, but proton-electron recombination occurs with greater frequency than atomic association, resulting in a larger atomic density in the steady-state. Time resolved plasma density data are needed to determine if the electron and neutral densities are equal on the time scale of the growth in the neutral density.

In addition to the evidence provided by the low neutral temperature, the temporal behavior of the neutral density also suggests a multi-step dissociation process. The neutral density data, in both the source and expansion regions, are well described by the equation

$$
n_{H}(t) \sim A(1-\exp (-B t))
$$

where both $A$ and $B$ are constants, and $t$ is the time in ms, see Figure 7.9. If dissociation is the result of molecular excitation, followed by dissociation, the rate equations for the ground state and excited molecules, and neutral atoms are

$$
\begin{aligned}
\frac{d n_{H_{2}}}{d t} & =-K_{e x c} n_{H_{2}} \\
\frac{d n_{H_{2}}^{*}}{d t} & =K_{d i s s} n_{H_{2}} n_{e}-K_{d i s s} n_{H_{2}}^{*} n_{e} \\
\frac{d n_{H}}{d t} & =K_{d i s s} n_{H_{2}}^{*} n_{e}
\end{aligned}
$$

where $K_{\text {exc }}$ and $K_{\text {diss }}$ are the rates of molecular excitation and dissociation. In the limit $K_{\text {exc }} \gg K_{\text {diss }}$, this is 


$$
\begin{aligned}
n_{H_{2}} & =n_{H_{2,0}} \exp \left(-K_{\text {exc }} t\right) \\
n_{H_{2}}^{*} & =n_{H_{2,0}} \frac{K_{\text {exc }}}{K_{\text {diss }}-K_{\text {exc }}}\left(\exp \left(-K_{\text {exc }} t\right)-\exp \left(-K_{\text {diss }} t\right)\right) \\
n_{H} & =n_{H_{2,0}}\left(1-\exp \left(-K_{\text {diss }} t\right) .\right.
\end{aligned}
$$

Thus, the time evolution of the neutral density displays the expected functional form for a multi-step dissociation process.

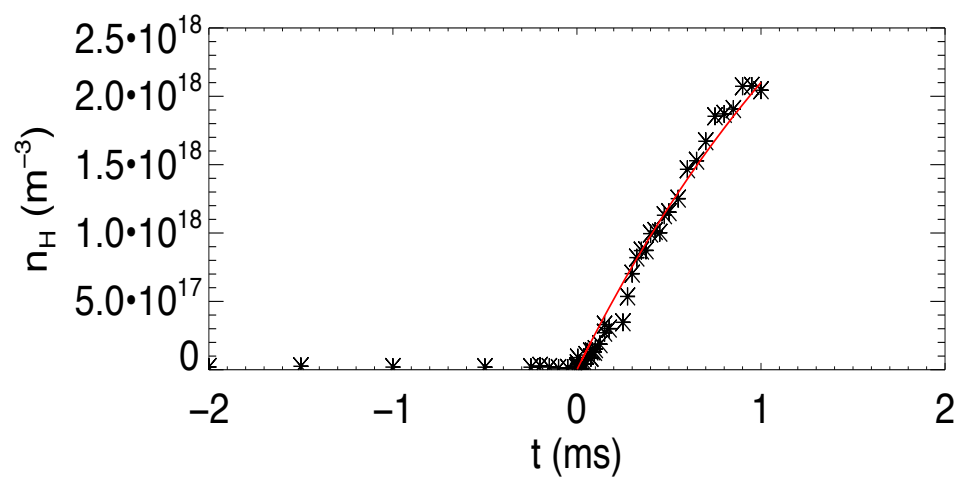

Figure 7.9: Time resolved neutral $\mathrm{H}$ density in the source region. Solid line is fit to $A(1-\exp (-B t))$, the function form expected for multistep dissociation.

In addition to fast time-resolved measurements, TALIF measurements made on a very long (hours) timescale, reveal a significant, 2-3x, growth in the neutral density in both hydrogen and deuterium, see Figure 7.10. The growth was measured following extended, 8 hour, Kr discharges. This behavior is consistent with wall loading in the expansion chamber. The Kr discharges effectively "clean" the walls in the expansion chamber. The walls are heated, forcing them to outgas. Such a technique is routinely used with helium in magnetic fusion devices. ${ }^{4}$ When $\mathrm{H}$ is run following $\mathrm{Kr}, \mathrm{H}$ neutrals are adsorbed into the "empty" stainless steel. Adsorption occurs up to a saturation point. As more neutrals are adsorbed, 
fewer neutrals adhere to the walls, increasing the density of neutrals in the plasma. The decrease in adsorption is consistent with an increase in neutral density for longer discharge times. The difference in wall loading saturation level and rate of wall loading between hydrogen and deuterium is consistent with the difference in density between the species. More deuterium neutrals in the plasma result in a larger fluence of atoms to the wall, resulting in faster wall loading. Wall loading studies such as these are very important for fusion devices, in which wall retention and dynamic release can affect plasma performance. Understanding how neutrals are retained and released from plasma facing walls provides insight into the recycling process.

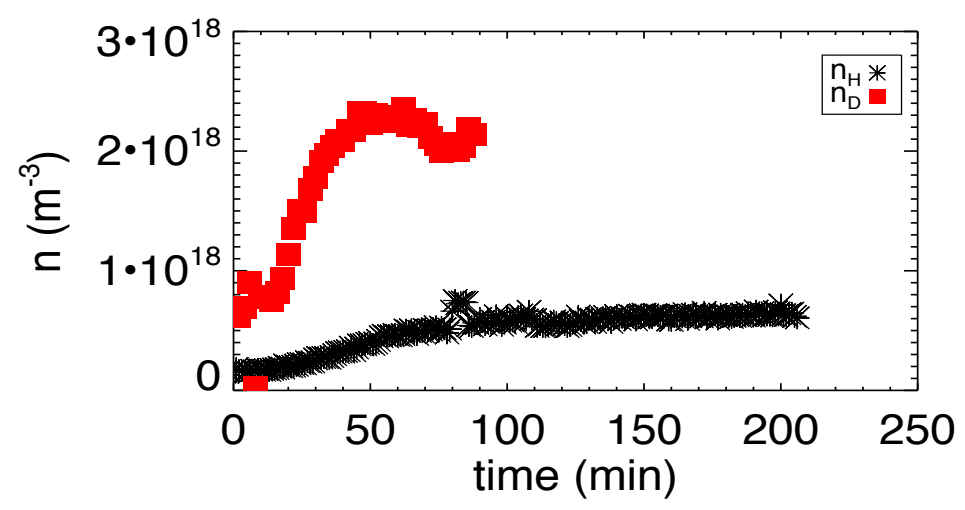

Figure 7.10: Atomic hydrogen (black) and deuterium (red) density versus time. Density ramp on long timescale is consistent with wall loading. 


\section{References}

[1] F. F. Chen, "Plasma ionization by helicon waves," Plasma Physics and Controlled Fusion, vol. 33, pp. 339-364, 1991.

[2] R. M. Magee, M. E. Galante, J. C. Jr., G. Lusk, D. W. McCarren, and E. E. Scime, "Neutral depletion and the helicon density limit," Submitted to Physics of Plasmas, 2013.

[3] H. Tawara, Y. Itikawa, H. Nishimura, and M. Yoshino, "Cross-sections and related data for electron collisions with hydrogen molecules and molecular ions," Journal of Physical and Chemical Reference Data, vol. 19, pp. 617-636, 1990.

[4] J. Ehrenberg, "Hydrogen and helium recycling in tokamaks with carbon walls," Journal of Nuclear Materials, vol. 162-164, pp. 63-79, 1989. 


\section{Chapter 8}

\section{Summary of Results and Future Work}

A new neutral density diagnostic based on two-photon absorption laser induced fluorescence (TALIF) was developed. The system represents an improvement on previous systems by combining, for the first time, very narrow laser line width with very high laser power. Three different injection/collection geometries were developed. The system was absolutely calibrated in a cold fill of neutral krypton gas. Using this diagnostic, ground state neutral densities and temperatures were measured in CW and pulsed krypton and hydrogen (deuterium) helicon plasma. The diagnostic has sufficient resolution that hydrogen and deuterium neutrals were resolvable in a mixed species discharge, see Figure 8.1. In a low temperature plasma, hydrogen and deuterium were resolvable using single-sided Doppler broadening injection geometry. In a high temperature tokamak, the neutral temperatures are expected to be sufficiently large to require Doppler-free excitation for isotope resolution. Calculations show that system performance is sufficient to measure the neutral density with an acceptable signal-to-noise ratio in the edge of a tokamak. 


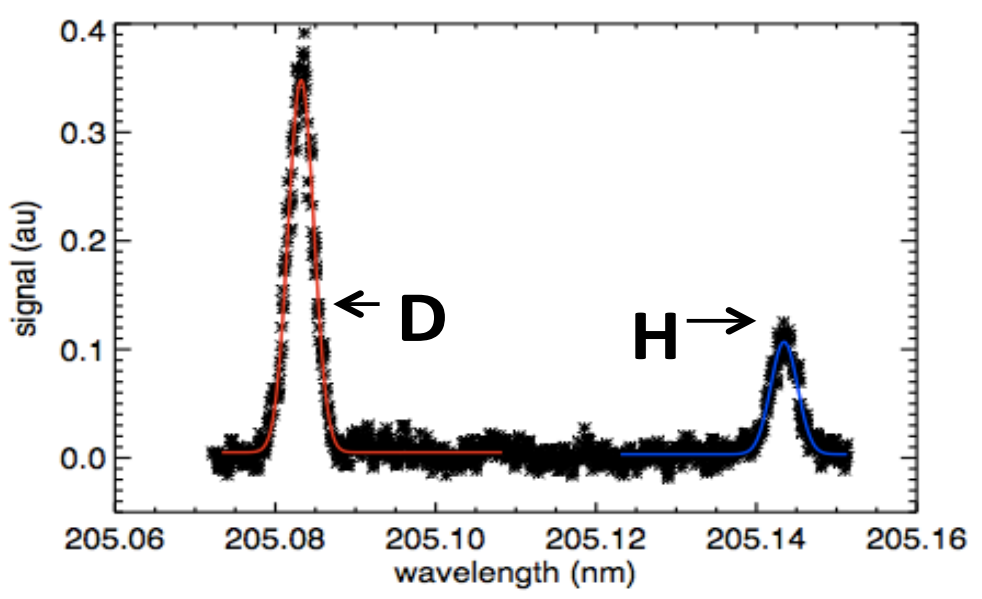

Figure 8.1: Spectrally resolved hydrogen and deuterium neutrals in a mixed species discharge.

TALIF measurements in a CW Kr plasma revealed significant neutral depletion when the source was operated in helicon mode. This neutral depletion resulted in an on-axis ionization fraction approaching 100\%, despite local plasma densities only $1 \%$ of the fill density. Additionally, neutral depletion placed an upper limit on the achievable plasma density. At high plasma densities, the neutrals were effectively screened out of the high plasma density region, starving the plasma of fuel. Time-resolved measurements in the same plasma showed that in the source region, the neutral depletion occurred on a very fast, 10 $\mu \mathrm{s}$, timescale. In the expansion region, the depletions occurred 1000x slower, $\sim 10 \mathrm{~ms}$. The difference in neutral loss between the two regions is consistent with diffusion out of the source and into the expansion region.

TALIF measurement in a CW H plasma revealed a very low level of dissociation, $~ 1 \%$. No neutral depletion was measured for any of the parametric configurations. Neutral profiles were relatively uniform radially, but displayed a considerable gradient axially, with the largest atomic densities in the source region. Additionally, the plasma density in $\mathrm{H}$ was considerably lower than in $\operatorname{Kr}(100 \mathrm{x})$ for the same source parameters. The most likely explanation for this 
is the sink for rf power presented by molecular dissociation. A 2-3x difference in both plasma and neutral density was measured between hydrogen and deuterium, with the deuterium densities everywhere larger than hydrogen. While a difference in plasma density has been previously observed, the difference in neutral density is unexpected and warrants further investigation.

Time resolved measurements in $\mathrm{H}$ showed that the atomic neutral density grew on the 100 $\mu \mathrm{s}$ timescale in both the source and expansion regions. It is unclear, due to insufficient time resolution, if the growth in the expansion region was due to neutral diffusion from the source or local dissociation. Sub $100 \mu$ s time resolved measurements are needed in the expansion region to answer this question. Neutral temperatures were low, $\sim 0.1 \mathrm{eV}$, in all parametric configurations. Time resolved measurements did not find a high temperature, 2 eV, population of neutrals, suggesting that neutral atoms were "born" with low energy through a multi-step dissociation process in which molecules were first excited, then dissociated.

Lastly, a long time scale, growth in neutral density was observed in both hydrogen and deuterium plasma following extend krypton discharges. This behavior is consistent with wall loading in the expansion chamber following cleaning discharges.

Future work should focus on achieving higher plasma densities in hydrogen so that helicon mode can be achieved. This could be accomplished by changing the antenna design to better couple power into the plasma. The Doppler-free technique should be refined. Doppler-free injection will be utilized in a magnetic fusion device due the desire to measure the entire neutral distribution within a single tokamak pulse, 1-5 s pulse length, and the need to resolve hydrogen isotopes. Additionally, with the Doppler-free technique, Stark splitting due to externally applied electric fields can be investigated. TALIF schemes using this laser system have been proposed for both helium ions and xenon neutrals. Helium ion 
spectroscopy is the "holy grail" of absorption spectroscopy. TALIF provides an avenue for achieving such a difficult measurement, and considerable effort should be put into it. Much like krypton, xenon provides an excellent species with which to study neutrals in high density helicon discharges. Unlike krypton, xenon has many accessible ion spectroscopic schemes. Coupling direct measurements of both neutrals and ions provides a wealth of information into many plasma phenomena, specifically, momentum transfer due to charge-exchange. As described at the end of Chapter 7, wall loading is a very important topic. Using TALIF, neutral retention and recycling can be directly measured. Further studies into wall loading, specifically neutral interactions with materials relevant to magnetic fusion devices, tungsten, lithium, etc., should be pursued in both hydrogen and deuterium. 


\section{Appendix A}

\section{CHEWIE Pulsing Scheme}

Time-resolved TALIF measurements are made by pulsing the plasma and scanning the laser in time with respect to the leading edge of the plasma pulse. The laser pulse is much shorter ( $8 \mathrm{~ns})$ than the typical plasma pulse (10s of ms), therefore, very high resolution can be achieved. In theory, the maximum time resolution is set my the decay time of the excited state, $\sim 50$ ns. In practice the maximum time resolution is determined by the electronics used in data acquisition and the need to collect a sufficient number of emitted photons for measurable signal. ${ }^{1}$ The pulsing system used to make time resolved TALIF measurements is outlined in this appendix.

\section{A.1 Triggering Scheme}

As previously stated, time resolved TALIF measurements were made by pulsing the plasma source and scanning the laser pulse across the plasma pulse. The triggering scheme is shown schematically in Figure A.1. 


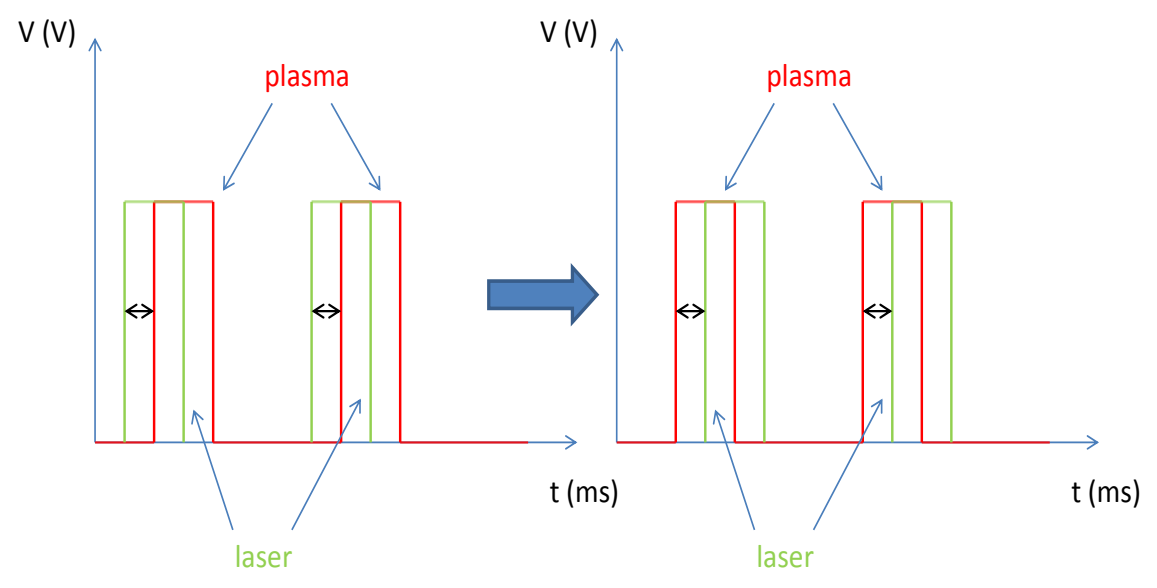

Figure A.1: Triggering scheme for pulsed TALIF measurements. Red is the plasma pulse, green is the laser.

Delaying the laser with respect to the plasma, or plasma with respect to the laser, is achieved with an analog delay generator (HP 8007B). An external function generator trigger both the Nd:YAG flash lamps and the delay generator. The laser must be triggered at 20 $\mathrm{Hz}$, thus the cadence of the measurement is held fixed. The laser flash lamps trigger the Q-switch, however there is a $100 \mu$ s delay between the lamp trigger and the Q-switch trigger. All synchronization must be done with respect to the Q-switch output to compensate for this delay. The delay generator triggers the plasma pulse, which is moved in time with respect to the Q-switch trigger by adjusted the delay generator. With this scheme a maximum time delay of $1 \mathrm{~ms}$ is achievable. To move the laser later into the plasma pulse the triggering must be flipped. The delay generator must trigger the flash lamps. When attempting to 
externally trigger the flash lamps the cadence cannot deviate from $20 \mathrm{~Hz}$ or the laser will not fire. 


\section{References}

[1] I. Biloiu, Laser Induced Fluorescence studies of ion acceleration in single and multiple species in expanding helicon plasma. PhD thesis, West Virginia University, 2009. 


\section{Appendix B}

\section{Dye Recipe}

TALIF laser dye recipe ${ }^{1}$

$\mathrm{R} 610-0.153 \mathrm{~g}$

R640 - $0.036 \mathrm{~g}$

Mix R610 and R640 in $900 \mathrm{~mL}$ of high purity methanol (ethanol) for 1-2 hours in electronic stirrer. Add $700 \mathrm{~mL}$ of dye mixture to resonantor/pre-amplifier dye cell. Add $1400 \mathrm{~mL}$ methanol (ethanol) to remaining $200 \mathrm{~mL}$ dye mixture. Add $1600 \mathrm{~mL}$ of diluted dye mixture to amplifier dye cell. 


\section{References}

[1] Sirah Dye Laser Manual. 